\title{
Feasibility of nSPECT for medical imaging
}

\author{
By \\ Adam Bell \\ B.Eng Aerospace (A), Carleton University 2009
}

A thesis submitted to the Faculty of Graduate and Postdoctoral Affairs in partial fulfillment of the requirements for the degree of

Master of Applied Science

in

Materials Engineering

Carleton University

Ottawa, Ontario

Copyright (C)

2011 - Adam Bell 
Library and Archives Canada

Published Heritage

Branch

395 Wellington Street Ottawa ON K1A ON4 Canada
Bibliotheqque et

Archives Canada

Direction du

Patrimoine de l'édition

395 , rue Wellington

Ottawa ON K1A ON4

Canada
Your file Votre référence

ISBN: 978-0-494-81673-8

Our file Notre référence

ISBN: 978-0-494-81673-8
NOTICE:

The author has granted a nonexclusive license allowing Library and Archives Canada to reproduce, publish, archive, preserve, conserve, communicate to the public by telecommunication or on the Internet, loan, distribute and sell theses worldwide, for commercial or noncommercial purposes, in microform, paper, electronic and/or any other formats.

The author retains copyright ownership and moral rights in this thesis. Neither the thesis nor substantial extracts from it may be printed or otherwise reproduced without the author's permission.
AVIS:

L'auteur a accordé une licence non exclusive permettant à la Bibliothèque et Archives Canada de reproduire, publier, archiver, sauvegarder, conserver, transmettre au public par télécommunication ou par l'Internet, prêter, distribuer et vendre des thèses partout dans le monde, à des fins commerciales ou autres, sur support microforme, papier, électronique et/ou autres formats.

L'auteur conserve la propriété du droit d'auteur et des droits moraux qui protège cette thèse. $\mathrm{Ni}$ la thèse ni des extraits substantiels de celle-ci ne doivent être imprimés ou autrement reproduits sans son autorisation.
In compliance with the Canadian Privacy Act some supporting forms may have been removed from this thesis.

While these forms may be included in the document page count, their removal does not represent any loss of content from the thesis.
Conformément à la loi canadienne sur la protection de la vie privée, quelques formulaires secondaires ont été enlevés de cette thèse.

Bien que ces formulaires aient inclus dans la pagination, il n'y aura aucun contenu manquant. 


\begin{abstract}
A novel technique (called nSPECT) is proposed for Single Photon Emission Computed Tomography (SPECT) imaging that replaces the conventional radioactive isotopes with Prompt Gamma Neutron Activated (PGNA) isotopes. The PGNA isotopes remain stable until they are activated by an external source of neutrons. This method has several advantages over traditional SPECT, including being able to restrict radiation dose to target tissue rather than over the entire body, which is currently the case, and removing the requirement for radioisotopes, including those made in nuclear reactors. Experiments with a commercial SPECT system were conducted to determine the feasibility of nSPECT for planar bone imaging. Planar images were acquired of simulated bone lesions in a plastic phantom containing ${ }^{131} \mathrm{I}$ as a proxy for a neutron-activated tracer. Estimated Compton scattering from concomitant hydrogen neutron-activation in the body was added to the ${ }^{131}$ I images to simulate the confounding effect of hydrogen activation. Simulated lesions with a $1 \mathrm{~cm}$ diameter could be seen after a 30 minute acquisition time. Discussions are included of patient doses from the pharmaceutical that might be used to deliver the isotope, and from the neutrons used to activate the isotope, both of which were found to be within acceptable limits.
\end{abstract}




\section{Acknowledgements}

I would like to acknowledge the contributions and support of the following people in helping to make this research a success: James Gräfe and the team from McMaster University for their work with samarium PGNAA, featured in this thesis; Marie-Christine Lépy and the researchers at the Laboratoire National Henri Becquerel (LNHB) in France for providing me with the Efficiency Transfer for Nuclide Activity measurements (ETNA) software for use on this project; My supervisor, Prof. Glenn McRae, for his essential advice and direction, who initiated this work along with Daniel Faber and myself; Dr. R. Glenn Wells of the University of Ottawa Heart Institute for his advice and Dr. Richard Wassenaar of The Ottawa Hospital for his advice and for his help in executing the research experiment. Finally, I would like to thank Heliocentric Technologies for funding this research. 


\section{Table of Contents}

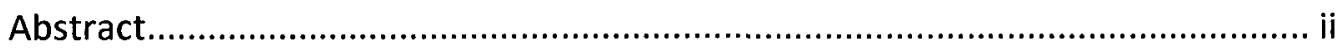

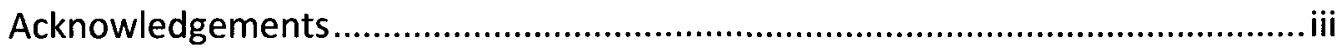

List of Tables....................................................................................................

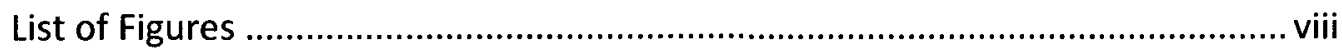

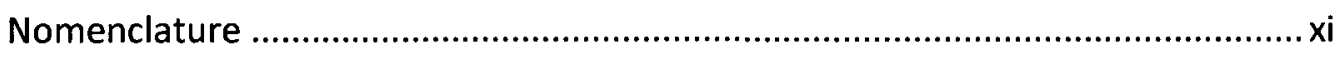

Chapter 1: INTRODUCTION .........................................................................

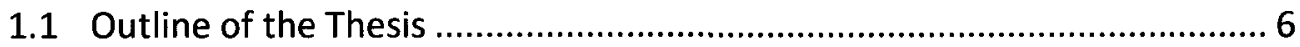

1.2 Identification of Contributions to the Research by the Author ................. 7

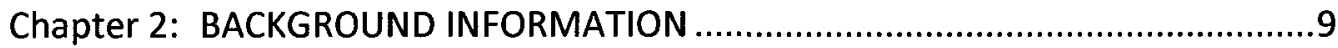

2.1 Introduction to Medical Imaging and SPECT ....................................... 9

2.2 A Brief History of the Development of Nuclear Medicine Imaging .......... 11

2.3 Modern Bone SPECT and Planar Imaging.......................................... 13

2.3.1 Tracer Molecules and Radioactive Isotopes .................................. 15

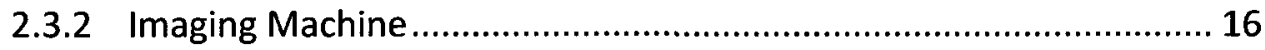

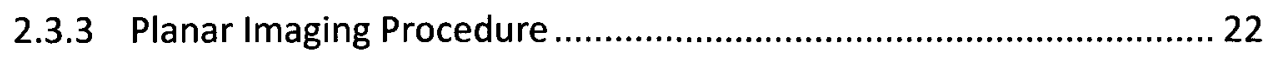

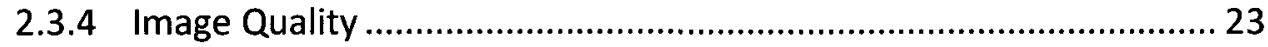

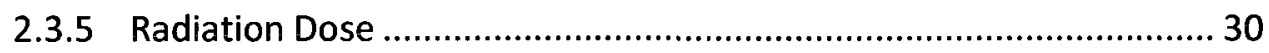

2.4 The Physics of SPECT and nSPECT Imaging ..................................... 33

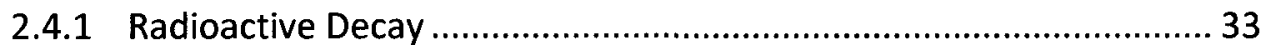

2.4.2 Gamma Radiation Interaction with Matter ................................... 36

2.4.3 Prompt Gamma Neutron Activation Analysis (PGNAA) ................... 39

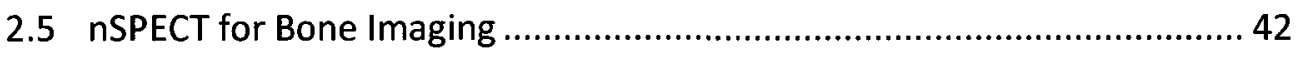

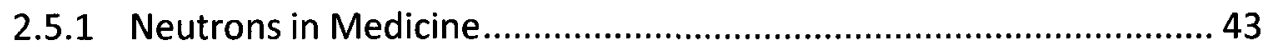

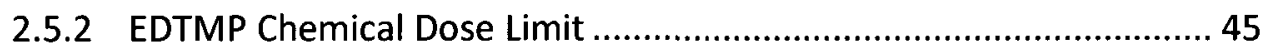

2.5.3 Potential Technical Obstacles .................................................. 47 
3.1 Summary of the Preliminary Experiment by McMaster University

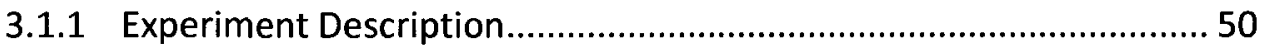

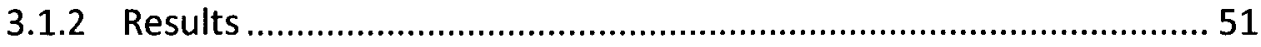

3.2 Objective of the ${ }^{131}$ I Experiment at The Ottawa Hospital ........................ 54

$3.3{ }^{131}$ I Experiment Description ........................................................... 55

3.4 Experimental Apparatus.................................................................... 56

3.4.1 Gamma Camera......................................................................... 57

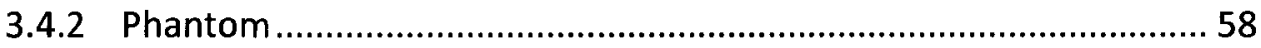

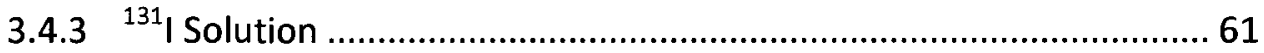

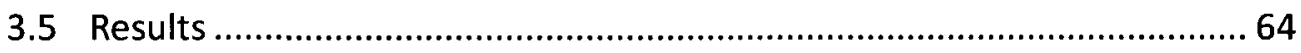

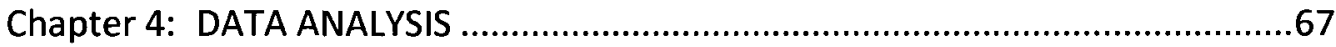

4.1 Hydrogen Noise and Patient Equivalent Dose .....................................67

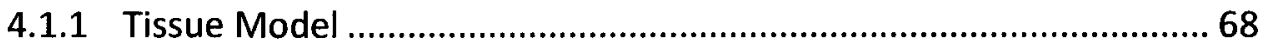

4.1.2 Neutron Flux Distribution in the Tissue Model .............................. 70

4.1.3 Compton Background from 2.2 MeV Photons................................ 71

4.1.4 Patient Equivalent Dose Rate from Neutrons ................................ 75

4.2 Adding Simulated Hydrogen Noise to the ${ }^{131}$ I Experiment Results........... 78

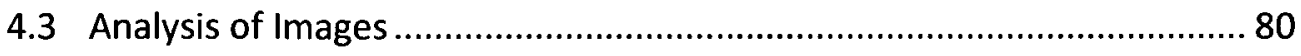

4.3.1 Compton-free Images (Unaltered ${ }^{131}$ I Phantom Images) .................. 85

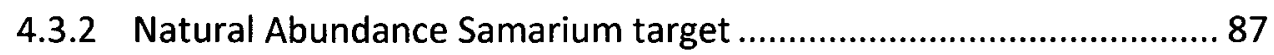

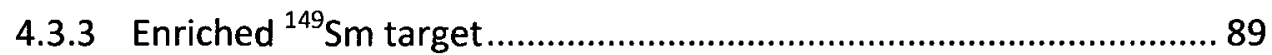

Chapter 5: RECOMMENDATIONS TO IMPROVE IMAGE QUALITY.......................92

Chapter 6: RECOMMENDATIONS FOR FUTURE EXPERIMENTS ….....................95

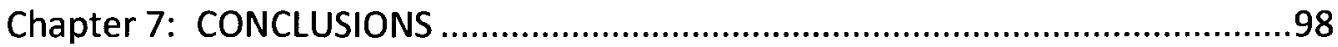

Appendix A - Calculations for EDTMP Oral Consumption ...............................101

Appendix B - Technical Drawings and Photos of the ${ }^{131}$ I Phantom.....................103

Appendix C - Phantom Hole Depth Attenuation Correction Calculation ............107 


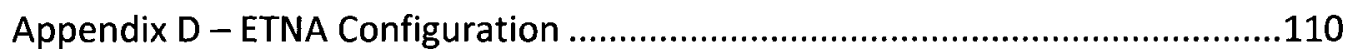

Appendix E - Signal Attenuation and Correction Factor for the Thigh Tissue

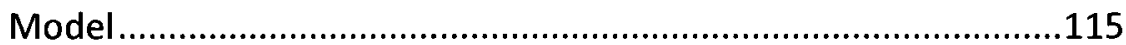

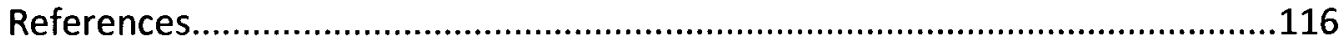




\section{List of Tables}

Table 1 - Radiation Weighting Factors ............................................................. 31

Table 2 - Isotopes and their neutron capture cross-sections.................................. 41

Table 3 - Radiation energies and emission probabilities for ${ }^{131}$ I........................... 61

Table 4 - Thigh tissue model parameters. ............................................................. 74 


\section{List of Figures}

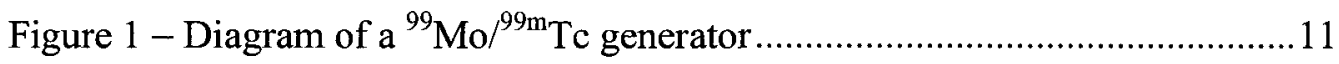

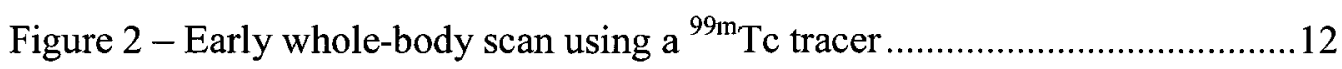

Figure 3 - Modern anterior and posterior planar bone images using ${ }^{99 \mathrm{~m}} \mathrm{Tc} . \ldots \ldots \ldots . .15$

Figure 4 - Diagram of a parallel-hole collimator...................................................18

Figure 5 - PMT array on a $\mathrm{NaI}(\mathrm{Tl})$ crystal ...........................................................2

Figure 6 - Example of the spectrum detected by a gamma camera from a

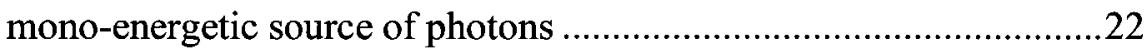

Figure 7 - Parallel hole collimator resolution (FWHM) for a point source...........27

Figure 8 - Lesion contrast reduction due to spatial resolution limitations ............29

Figure 9 - Photon linear attenuation coefficient versus photon energy for

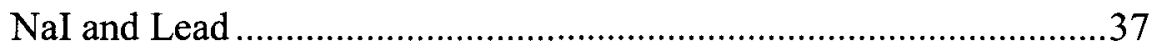

Figure 10 -Illustrating the process of prompt gamma neutron activation .............41

Figure 11 - An illustration of the nSPECT system concept .................................42

Figure 12 - Total ${ }^{149} \mathrm{Sm}$ mass deposited on the skeleton from a daily oral

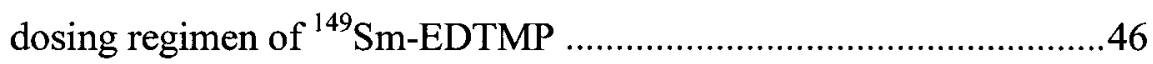

Figure 13 - Diagram of the McMaster Samarium PGNA experiment ………........51

Figure 14 - Plot of neutron capture rate versus Sm concentration in the 125

$\mathrm{mL}$ phantom for the McMaster Experiment..........................................52

Figure 15 - Photo of the Siemens Symbia-T at The Ottawa Hospital ....................58

Figure 16 - Isometric, front, and top views of the phantom .................................59

Figure 17 - Diagram illustrating lesion contrast in the ${ }^{131}$ I phantom ....................60 viii 
Figure 18 - Images of the ${ }^{131}$ I phantom produced by the Symbia T SPECT system

Figure 19 - Images of the ${ }^{131}$ I phantom for a 2-minute acquisition time with reduced matrix sizes $(64 \times 64$ and $128 \times 128)$ .66

Figure 20 - Thigh tissue model for calculating Compton noise and patient radiation dose rate

Figure 21 - MCNP5 simulation data for a fast neutron source surrounded by concentric spheres of HDPE .70

Figure $22-2$-minute ${ }^{131} \mathrm{I}$ image combined with the $2.2 \mathrm{MeV}$ Compton counts from the two $\mathrm{Sm}$ tracer scenarios 80

Figure $23-{ }^{131}$ I phantom image showing the region used to calculate $B_{\text {Avg }} \ldots \ldots \ldots . .82$

Figure 24 - Example of 1-D filtering with a three-point binomial filter

Figure 25 - A plot of lesion $\mathrm{SNR}_{\text {Rose }}$ against image acquisition time for the

${ }^{131}$ I phantom images 86

Figure 26 - Filtered ${ }^{131} \mathrm{I}$ images simulating 10-minute acquisitions for natural abundance and enriched Sm tracers 86

Figure 27 - A plot of lesion SNR $_{\text {Rose }}$ against image acquisition time for the simulated natural abundance Sm tracer images 88

Figure 28 - Scatter subtracted and filtered simulated images of 10-minute and 30-minute acquisitions for a natural abundance $\mathrm{Sm}$ tracer 88

Figure 29 - A plot of lesion $\mathrm{SNR}_{\text {Rose }}$ against image acquisition time for the simulated enriched ${ }^{149} \mathrm{Sm}$ tracer images. 
Figure 30 - Scatter subtracted and filtered simulated images of 10-minute and 30-minute acquisitions for an enriched ${ }^{149} \mathrm{Sm}$ tracer..................91

Figure 31 - A plot showing an example of acceptance energy window

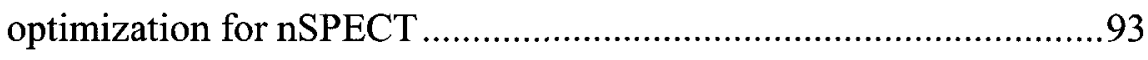




\section{Nomenclature}

\section{Abbreviations}

ALARA

BNCT

$\mathrm{Bq}$

EDTMP

ETNA

eV

FEPE

FOV

FWHM

Gy

HDPE

HE

HPGe

MCNP

MDP

MNR

NaI(Tl)

nSPECT

PGNA(A)

PMT

ppm

PSF

PuBe

ROC

SNR

SPECT

$\mathrm{Sv}$
As Low As Reasonably Achievable

Boron Neutron Capture Therapy

Becquerel, unit of Activity [ $\mathrm{s}^{-1}$ ]

Ethylenediamine tetra(methylene phosphonic acid)

Efficiency Transfer for Nuclide Activity measurement

Electronvolt, unit of energy $\left[\sim 1.602 \times 10^{-19} \mathrm{~J}\right]$

Full Energy Peak Efficiency

Field Of View

Full Width at Half Maximum

Gray, unit of radiation dose $[\mathrm{J} / \mathrm{kg}]$

High Density Polyethylene

High Energy

High-Purity Germanium

Monte Carlo N-Particle code

Methylene diphosphonate

McMaster Nuclear Reactor

Thalium-doped sodium iodide

Neutron-activated Single Photon Emission Computed Tomography

Prompt Gamma Neutron Activation (Analysis)

Photomultiplier Tube

Parts-per-million

Point Spread Function

Plutonium-Beryllium neutron source

Receiver Operating Characteristic

Signal to Noise Ratio

Single Photon Emission Computed Tomography

Sievert, unit of equivalent and effective radiation dose [J/kg* weighting factors] 


\section{Symbols}

\begin{tabular}{|c|c|}
\hline$a_{T}$ & Area of a target in an image \\
\hline$n_{s}$ & Mean photon density of the signal of a target in an image (photons/unit area) \\
\hline$n_{b}$ & $\begin{array}{l}\text { Mean photon density of the background of a target in an image (photons/unit } \\
\text { area) }\end{array}$ \\
\hline$R_{C}$ & Collimator resolution \\
\hline$h$ & Collimator septa thickness \\
\hline$d$ & Collimator hole diameter \\
\hline$l$ & Collimator hole length \\
\hline$z$ & Source-to-collimator distance \\
\hline$b$ & Scintillator crystal thickness \\
\hline$t_{1 / 2}$ & Physical half-life of a radioactive isotope \\
\hline$\Delta N$ & Mean number of decays from a group of radioactive isotopes \\
\hline$N$ & Number of atoms \\
\hline$\Delta t$ & Time interval \\
\hline$\lambda$ & Transformation constant for a radioactive isotope \\
\hline$A$ & Activity, the number of radioactive decays per second \\
\hline$\mu$ & Linear attenuation coefficient \\
\hline$\varphi$ & Neutron flux \\
\hline$\sigma$ & Neutron capture cross-section of an isotope \\
\hline$m$ & Mass \\
\hline$C$ & Concentration by mass \\
\hline$M$ & Molar mass \\
\hline$N_{A}$ & Avogadro's number \\
\hline$a$ & Unit area \\
\hline$\rho$ & Density \\
\hline$d_{\text {bone }}$ & Thickness of bone \\
\hline$V$ & Volume \\
\hline$c_{i}$ & Total number of detector counts in pixel $i$ \\
\hline$B_{\text {Avg }}$ & Average number of background detector counts per pixel \\
\hline$K$ & Total number of pixels comprising a lesion in an image \\
\hline
\end{tabular}




\section{CHAPTER 1: INTRODUCTION}

In May of 2009, the National Research Universal reactor (NRU) in Chalk River, Ontario, was unexpectedly shutdown after the discovery of a heavy water leak (1), leading to a worldwide shortage of the ${ }^{99} \mathrm{Mo}$ isotope. Prior to this shutdown, the NRU reactor was responsible for approximately $33 \%$ of the world's supply of ${ }^{99} \mathrm{Mo}$, from which the medical isotope ${ }^{99 m} \mathrm{Tc}$ is produced (1). Technetium-99m is used in approximately $80 \%$ of the 46 million nuclear medicine tests performed every year worldwide (2). Approximately 2 million of those tests are performed in Canada at an estimated market value of $\$ 40$ million annually for the radiopharmaceuticals alone $(2 ; 3)$. The isotope shortage created by the NRU shutdown was compounded when the High Flux Reactor (HFR) in Petten, Netherlands (accounting for $32 \%$ of the world's ${ }^{99} \mathrm{Mo}$ isotope supply) was shut down for a few weeks during the summer of 2009 , and then for another 6 months starting in February 2010 (1). The NRU was eventually re-started in August 2010, and the HFR resumed production in September 2010, ending a 16-month isotope shortage, which included 7 months when the world was without two-thirds of its ${ }^{99}$ Mo isotope production (4).

The return to full production may be only temporary, however. The average age of the five reactors that provide the majority of the world's ${ }^{99}$ Mo production is 47 years, and the 53 year-old NRU is scheduled to cease all production in $2016(1 ; 2)$. The NRU was intended to be replaced by two Multipurpose Applied Physics Lattice Experiment 
(MAPLE) reactors, developed by Atomic Energy of Canada Limited and MDS Nordion, however, their development was halted in 2008 (5).

Throughout the isotope crisis, Nuclear Medicine staff attempted to compensate for the lack of ${ }^{99 \mathrm{~m}} \mathrm{Tc}$. Rationing was not an option because of the short physical half-lives of ${ }^{99}$ Mo (66 hours) and ${ }^{99 m}$ Tc (6 hours) (6). CT and MRI can be used in a limited number of cases, as well as isotopes that are produced from cyclotrons, such as ${ }^{201} \mathrm{Tl}$ (which was replaced by ${ }^{99 m}$ Tc $15-20$ years ago) and Positron Emission Tomography (PET) isotopes $(2 ; 6)$. However, each of these alternatives is either more expensive, less accurate, or provides a higher patient radiation dose, which is why they are typically not used clinically when ${ }^{99 m} \mathrm{Tc}$ is available (6). These alternatives helped mitigate the impact of the ${ }^{99 \mathrm{~m}} \mathrm{Tc}$ shortage, but quality of care still suffered (7).

A study by the Society of Nuclear Medicine, which was designed to assess the impact of the ${ }^{99 \mathrm{~m}} \mathrm{Tc}$ shortage, indicated that $92 \%$ of the medical facilities polled reported that they had to cancel or postpone Single Photon Emission Computed Tomography (SPECT) imaging procedures employing ${ }^{99 m} \mathrm{Tc}(7)$. Approximately one-third of medical facilities were only able to operate at $50 \%-75 \%$ of their normal capacity, and this study was conducted before the HFR reactor was shut down for maintenance for 6 months in 2010 (7). In the case of cancer treatment in particular, these delays or cancellations can have very negative impacts on a patient's treatment and chances of survival (7). For example, in treating metastatic cancers, ${ }^{99 \mathrm{~m}} \mathrm{Tc}$ bone scans are required every 6-8 weeks to determine whether a particular treatment regimen is successful or whether a new 
approach is required (7). Delays can result in cancers progressing unchecked by a treatment plan that is discovered to be ineffective too late (7).

In response to the seriousness of the shortage, governments have committed significant resources to finding both short- and long-term solutions. The total cost of repair for the NRU (including lost revenue from isotopes) is estimated at $\$ 70$ million (8). The American Medical Isotopes Production Act was introduced in 2009, authorizing a $\$ 163$ million Department of Energy program to support domestic production of ${ }^{99} \mathrm{Mo}$, of which the United States currently has none (4). In Canada, in June of 2009, the Minister of Health with the Canadian Institutes of Health Research (CIHR) and the Natural Sciences and Engineering Research Council of Canada (NSERC) announced \$6 million in funding for research into alternatives to nuclear reactor ${ }^{99} \mathrm{Mo} /{ }^{99 \mathrm{~m}} \mathrm{Tc}$ production (9). Following this commitment, Natural Resources Canada initiated a 2-year, \$35 million program to advance cyclotron and linear accelerator technology for producing ${ }^{99 \mathrm{~m}} \mathrm{Tc}(10)$.

There are currently two promising proposals for alternative methods of producing ${ }^{99}$ Mo or ${ }^{99 m} \mathrm{Tc}$ : using a high-power electron accelerator with the ${ }^{100} \mathrm{Mo}$ (photon, $\mathrm{n}$ ) ${ }^{99} \mathrm{Mo}$ reaction or the cyclotron approach with the ${ }^{100} \mathrm{Mo}(\mathrm{p}, 2 \mathrm{n})^{99 \mathrm{~m}} \mathrm{Tc}$ reaction $(10 ; 3)$. The accelerator-based method would produce ${ }^{99} \mathrm{Mo}$ in a centralized facility, and the ${ }^{99} \mathrm{Mo}$ would then be shipped to the United States, where it would be manufactured into ${ }^{99 \mathrm{~m}} \mathrm{Tc}$ generators, similar to what is currently done today (3). The accelerators and associated facilities required to meet the Canadian demand are estimated to cost between $\$ 34$ million and $\$ 64$ million and could be available between 2013 and 2015, though there is 
still significant $R \& D$ required before this method becomes commercial (3). In the cyclotron approach, ${ }^{99 \mathrm{~m}} \mathrm{Tc}$ would be produced directly from ${ }^{100} \mathrm{Mo}$ by bombarding it with protons, which causes two neutrons to be emitted (3). This approach necessitates that the cyclotron be housed in or near the hospital or clinic, since ${ }^{99 \mathrm{~m}} \mathrm{Tc}$ is short-lived; therefore, this option would not be cost-effective for supplying rural demand so it is not a standalone solution (3). The R\&D cost is expected to be $\$ 5$ million and the cyclotrons are estimated to cost $\$ 5$ million to $\$ 9$ million each and to be available at some time between 2011 and 2014 (3). Unfortunately, the commercial viability of both the linear accelerator and cyclotron alternatives is dependent on the availability and cost of ${ }^{100} \mathrm{Mo}$, which is still unknown (3).

A novel approach to the issue of radioisotope production for SPECT imaging is presented in this work. To date, SPECT isotopes are derived from nuclear reactors or cyclotrons and chemically bound to a tracer molecule and injected into a patient (11). The method proposed in this thesis is a paradigm shift for SPECT imaging: Instead of producing a radioactive isotope and then injecting it into a patient for imaging, a stable isotope is injected into a patient first and then stimulated to emit radiation in-vivo.

The method is christened neutron-activated SPECT (nSPECT). The recent development of compact, high-output accelerator-based neutron sources that do not require radioactive fuel has made in-vivo neutron activation of tracer isotopes possible (12). A neutron generator of this type would be incorporated into a SPECT imaging system to provide the means of stimulus for the stable tracer isotopes. The stable tracer 
isotopes emit gamma rays for SPECT imaging through the Prompt Gamma Neutron Activation (PGNA) reaction; once activated by external neutron radiation, the tracer isotopes decay instantly (13). nSPECT could provide the same information as current SPECT while potentially allowing unprecedented control of the tracer activity in a patient. Additionally, the stable tracer isotopes do not have a physical shelf-life, and can be stockpiled to hedge against manufacturer shortages.

nSPECT is designed to achieve better supply stability, enhanced safety, and improved accessibility in diagnostic nuclear medicine. Because accelerator-based neutron sources are electronic and neutron beams can be pulsed, filtered and collimated, the tracer activity in a patient can be restricted to a target region and switched "on" or "off" on command. Unlike current SPECT techniques, where a patient receives a whole-body dose from radiopharmaceuticals, patient dose in nSPECT is limited to the region being imaged (11). With radiation exposure confined to a single controlled space (i.e., the immediate area around the nSPECT imaging system) over a limited period of time, nSPECT could enhance the safety of technicians, patients and physicians. Because nSPECT isotopes do not have limiting physical half-lives and the neutron sources are relatively small, it is equally tractable in rural or urban operation, in large hospitals or small clinics, making nSPECT more accessible than ${ }^{99 \mathrm{~m}}$ Tc SPECT may be in the near future. 


\subsection{Outline of the Thesis}

This Thesis is the first report of research on the subject of neutron-activated tracers for nuclear medicine diagnostic imaging. The goal of this work was to conduct a preliminary assessment of the feasibility of the nSPECT method. To determine whether nSPECT may be feasible, three parameters were assessed: patient tracer dose, radiation equivalent dose rates, and image quality. Where appropriate, the values of these parameters were compared with those of modern nuclear medicine imaging. A specific procedure, planar bone imaging, was examined since it is one of the two most frequent SPECT procedures, alongside myocardial perfusion imaging (6).

Following this introduction, a literature review of the SPECT imaging system and method, the physics of radioactive decay, and neutron interactions is provided. A more detailed overview of the nSPECT concept, specifically its application for planar bone imaging, is also provided. Subsequent chapters contain the details of the experiment design, results, analyses and the conclusions on the feasibility of nSPECT, as well as recommendations for improvements to the method and suggestions for future experiments. The primary experiment involved using a commercial SPECT system to image a phantom containing the ${ }^{131} \mathrm{I}$ radioisotope as a proxy for a neutron activated isotope that would be used for nSPECT. These ${ }^{131} \mathrm{I}$ images were used to generate simulated images of what might be produced using nSPECT. Preliminary tracer and radiation dose estimates for nSPECT imaging are also provided. 


\subsection{Identification of Contributions to the Research by the Author}

The general concept of nSPECT was conceived by Dr. Glenn McRae, the supervisor and mentor of the author. The author was the major contributor to the subsequent research and detailed design of the nSPECT method, including the selection of the target isotope and carrier molecule $\left({ }^{149} \mathrm{Sm}-\mathrm{EDTMP}\right)$.

The material in Section 2.5 was developed primarily by the author through various analyses, simulations, and research, with guidance from Dr. Glenn McRae. This work was the foundation for the two experiments discussed in this thesis. The first experiment, described in Section 3.1, was initiated by the author after contacting the group at McMaster University. This experiment was very similar to previous experiments that had been done at McMaster University, so the experiment design effort by the author was minimal. Our collaborators at McMaster University performed the experiment and provided the author with the results. The McMaster experiment data were used in a simulation, designed and executed by the author, the results of which were used to design the second experiment, The ${ }^{131}$ I Experiment.

The ${ }^{131}$ I Experiment, described in Section $3.2-3.5$, was designed by the author, with input from Dr. Glenn McRae. Dr. Glenn Wells suggested that ${ }^{131}$ I could be used as a proxy for ${ }^{149} \mathrm{Sm}$ PGNA. Dr. Richard Wassenaar assisted with the ${ }^{131}$ I Experiment at The Ottawa Hospital. 
The analyses presented in Chapter 4: were designed and executed by the author, with guidance from Dr. Glenn McRae. Dr. Richard Wassenaar and Dr. Glenn Wells provided important feedback on the results of these analyses. The recommendations for improving nSPECT in Chapter 5: were developed by the author. The recommendations for future experiments in Chapter 6: were developed by both the author and Dr. Glenn McRae.

Two conference papers, written by the author, based on this thesis research, have been published:

1) NEUTRON ACTIVATION FOR PLANAR AND SPECT IMAGING. Bell, Adam R.; McRae, G.; Wassenaar, R.; Wells, G. Chicago : IEEE, 2011. International Symposium on Biomedical Imaging (ISBI). pp. 1801-1804. Poster presentation.

2) Neutron Activation for Planar and SPECT Imaging: Locating Metastatic Bone Lesions in the Axial Skeleton. Bell, Adam R.; McRae, G.; Wassenaar, R.; Wells, G. Niagara Falls : CNS-CNA, 2011. Canadian Nuclear Society (CNS) 32nd Annual Conference. Paper accepted, to be published in conference proceedings in June 2011 and presented as a poster. 


\section{CHAPTER 2: BACKGROUND INFORMATION}

This chapter contains the relevant methods, concepts, and physics that form the basis for the experimental results and analyses presented in this thesis. It also provides context for how this research could complement or replace SPECT and single photon emission planar imaging.

\subsection{Introduction to Medical Imaging and SPECT}

Medical imaging is a non-invasive method of obtaining anatomical or functional information about a patient. Anatomical information is the spatial distribution of various types of tissues in the body. Imaging systems that provide anatomical information are $\mathrm{X}$ ray projection and Computed Tomography (CT), Magnetic Resonance Imaging (MRI), and Ultrasound imaging (14). Each method provides anatomical information using a different information carrier; for example, in CT, tissues are located and differentiated according to their density and quantity of heavier elements (like $\mathrm{Ca}, \mathrm{K}, \mathrm{Fe}$, etc.), whereas in MRI, tissues may be differentiated according to their hydrogen content (14). Functional information is any information that describes a physiological process, such as blood flow through the heart or the rate of bone growth. Imaging systems that yield this type of information are Positron Emission Tomography (PET) and Single Photon Emission Computed Tomography (SPECT) (14). PET and SPECT functional imaging consists of injecting radioactive isotopes into the body and locating them with the imaging system as they participate in some physiological process (14). Different 
physiological processes are imaged with radioisotopes attached to different processspecific radiopharmaceuticals (14).

The cornerstone of SPECT imaging is the ${ }^{99 \mathrm{~m}} \mathrm{Tc}$ isotope. It accounts for over $80 \%$ of all SPECT scans and is used in imaging lungs, kidneys, brain, heart, thyroid and in whole body bone scans $(15 ; 2)$. It can be chemically attached to a variety of molecules and it has a gamma ray emission energy $(140 \mathrm{keV})$ within the ideal range, balancing patient dose and image quality (16). It has a relatively short physical half-life ( $\sim 6$ hours) which helps to limit the total radiation dose to the patient, but it is produced from ${ }^{99} \mathrm{Mo}$, a much longer-lived isotope ( $\sim 66$ hours), through radioactive decay in a small portable generator. It is feasible to transport the generator over large distances because of the longer physical half-life of ${ }^{99} \mathrm{Mo}$, which ensures that a significant amount of ${ }^{99}$ Mo still remains after the generator arrives at its destination (16). Figure 1 is an example of a commercial ${ }^{99} \mathrm{Mo} /{ }^{99 m} \mathrm{Tc}$ generator and its basic internal design and function. The ${ }^{99} \mathrm{Mo} /{ }^{99 \mathrm{~m}} \mathrm{Tc}$ generator is made by loading ${ }^{99} \mathrm{Mo}$ onto aluminum oxide columns, which bind ${ }^{99}$ Mo with a higher chemical affinity than ${ }^{99 \mathrm{~m}} \mathrm{Tc}$ (17). To obtain ${ }^{99 \mathrm{~m}} \mathrm{Tc}$ from the generator, the column is washed with a saline solution and the ${ }^{99 \mathrm{~m}} \mathrm{Tc}$ is removed from the column as sodium pertechnetate, while ${ }^{99}$ Mo remains on the column (17). 


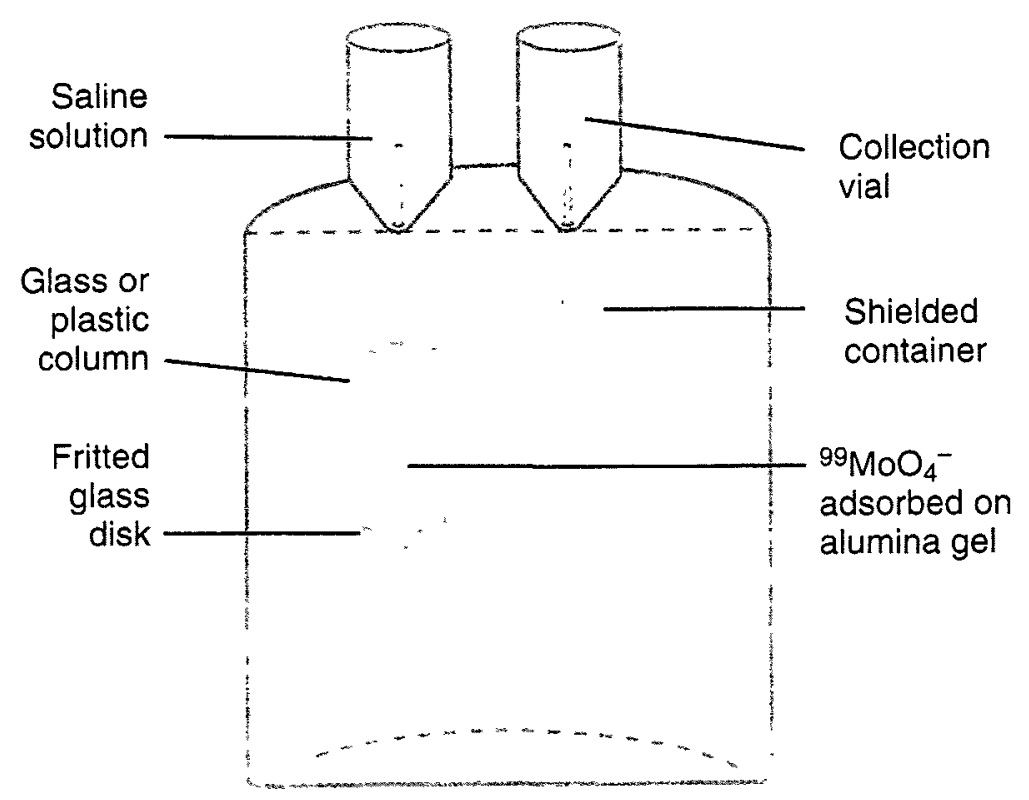

Figure 1 - Diagram of the internal components of a ${ }^{99} \mathrm{Mo} /{ }^{99} \mathrm{mTc}$ generator ${ }^{1}$

\subsection{A Brief History of the Development of Nuclear Medicine Imaging}

Nuclear medicine imaging began with the use of radioiodine $\left({ }^{131} \mathrm{I}\right)$ in the late 1940's to treat thyroid disease (11). Scintillation detectors were soon employed to help assess ${ }^{131} \mathrm{I}$ uptake and to diagnose the size and distribution of the lesions (11). Hal Anger's development of the Anger Gamma Camera (the arrangement of a scintillator crystal with multiple photomultiplier tubes for spatial resolution) in the late 1950's is considered the most significant discovery/invention responsible for the emergence of

${ }^{1}$ This figure was published in Nuclear Medicine, $2^{\text {nd }}$ ed., Vol. 1, Augustine, S. C.; Rutar, F. J.; Holdeman, K. P.; Jacobson, D. A.; Dalrymple, G. V., Chapter 26: Radionuclide Generators: Design, Operation, and Clinical Utility, Page 376, Copyright Elsevier (2006), reproduced with permission. 
nuclear medicine (11). The Anger Camera replaced the older rectilinear scanning detectors, and prepared the path for future developments in planar imaging and eventually SPECT imaging (11). Paul Harper helped pioneer the use of ${ }^{99 m}$ Tc for medical imaging after producing the world's first whole-body image using a ${ }^{99 \mathrm{~m}} \mathrm{Tc}$ tracer in 1965 (see Figure 2) (18). The first commercial technetium generator was also sold in 1965 and in the following years a variety of ${ }^{99 \mathrm{~m}} \mathrm{Tc}$ labeled compounds emerged for use in nuclear medicine imaging (11).

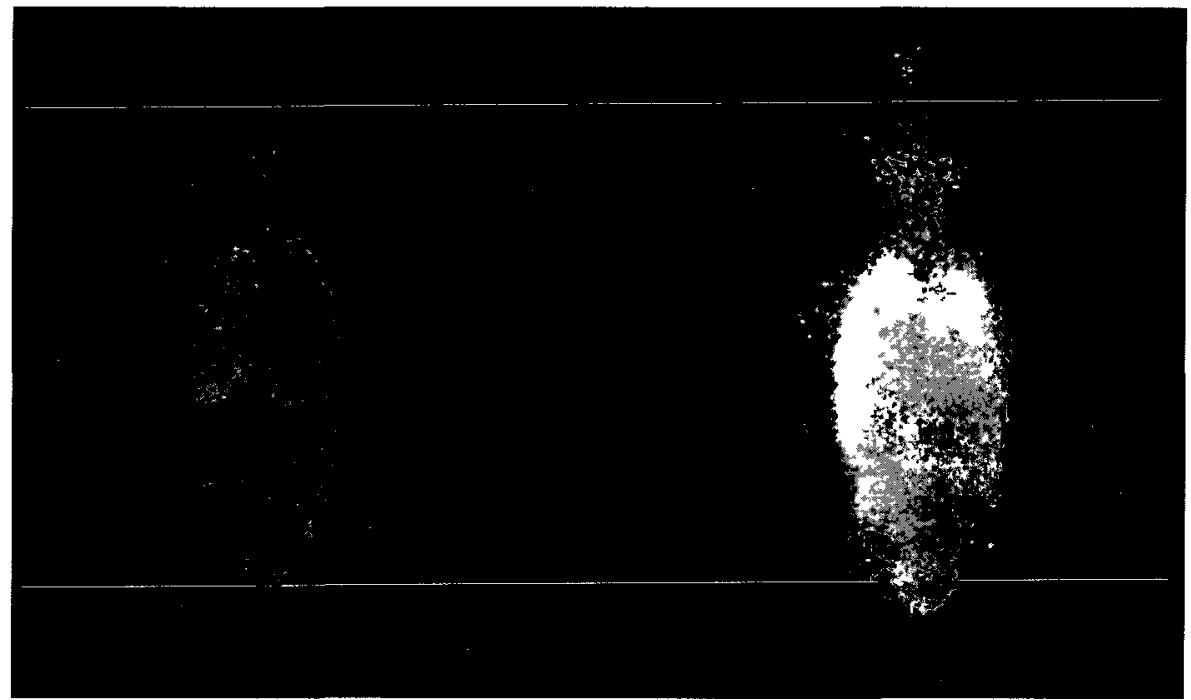

Figure 2 - Whole-body scan of a 6 year-old child 1 hour after a $93 \mathrm{MBq}(2.5 \mathrm{mCi})$ injection of a ${ }^{99 m} \mathrm{Tc}$ tracer. Each image represents a 2-3 minute integration time. The image on the left (anterior scan) shows the lungs, liver, spleen, stomach and bladder. The image on the right (posterior scan) is over-exposed to permit a better demarcation of the soft tissue of the arm, base of the skull, and sagital sinus. ${ }^{2}$

${ }^{2}$ Reprinted from Elsevier Academic Press, $1^{\text {st }}$ ed., Wernick, Miles N.; Aarsvold, John N., "Evolution of Clinical Emission Tomography," Pages 25-52, Copyright (2004), with permission from Elsevier. 


\subsection{Modern Bone SPECT and Planar Imaging}

Planar bone imaging is currently the most sensitive method of whole-body skeletal imaging and it accounts for approximately 17\% of all SPECT imaging procedures using ${ }^{99 \mathrm{~m}} \mathrm{Tc}(15 ; 3)$. To accomplish the whole-body bone scan, a patient is injected with a ${ }^{99 \mathrm{~m}} \mathrm{Tc}$-labeled phosphate or phosphonate molecule, typically ${ }^{99 \mathrm{~m}} \mathrm{Tc}-\mathrm{MDP}$ (methylene diphosphonate) (19). A large fraction of the initial injection is absorbed into the mineral phase of bone within one hour, and the patient is usually imaged with a SPECT camera between 2 and 24 hours after injection (19). The SPECT camera detects the radiation emitted by the ${ }^{99 \mathrm{~m}} \mathrm{Tc}$ isotope and, for every photon detected, calculates its point of origin on the detector plane. The accumulation of hundreds of thousands of detected photons and their spatial distribution is used, in the case of a planar scan, to produce anterior and posterior images (see Figure 3). An increased uptake of the molecule is seen as a dark spot in the image. Dark spots in bone scan images can result from increased blood flow to lesions and an increase in osteoblastic (bone growth) activity (19). The bladder can also appear as a dark area due to an accumulation of unabsorbed ${ }^{99 \mathrm{~m}} \mathrm{Tc}$ that has been removed from the blood stream by the kidneys (19). Clinical applications of planar bone imaging can be divided into two categories (19): 
Neoplastic (Cancer):

- Primary Tumors - Pre and post therapy examination, evaluation of therapy response

- Secondary Tumors (metastases) - Pre and post therapy examination, distribution of osteoblastic activity prior to radiotherapy $\left({ }^{153} \mathrm{Sm}\right.$-EDTMP, ${ }^{186} \mathrm{Re}-\mathrm{HEDP}$, etc. $)$

Non-Neoplastic:

Planar bone imaging can be used to help diagnose the following conditions:

- Osteomyelitis

- Perthe's Disease, Avascular necrosis

- Metabolic disorders (Paget, osteoporosis)

- Arthropathies

- Fibrous Dysplasia and other rare congenital conditions

- Stress fractures, shin splints

- Loose or infected joint prosthesis

- Sacroilitis (low back pain)

- Reflex sympatetic syndrome

- Many other bone injuries 


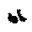

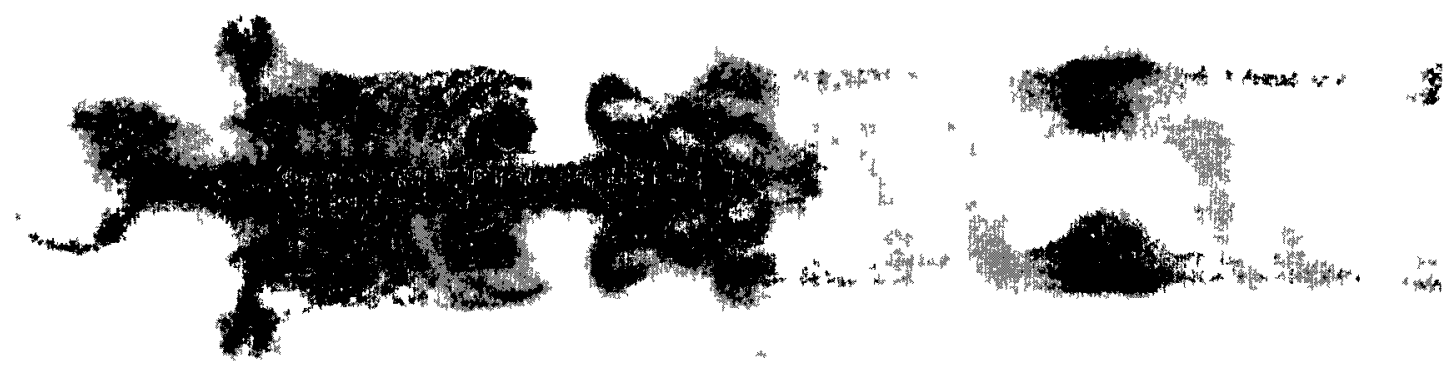

$\infty$

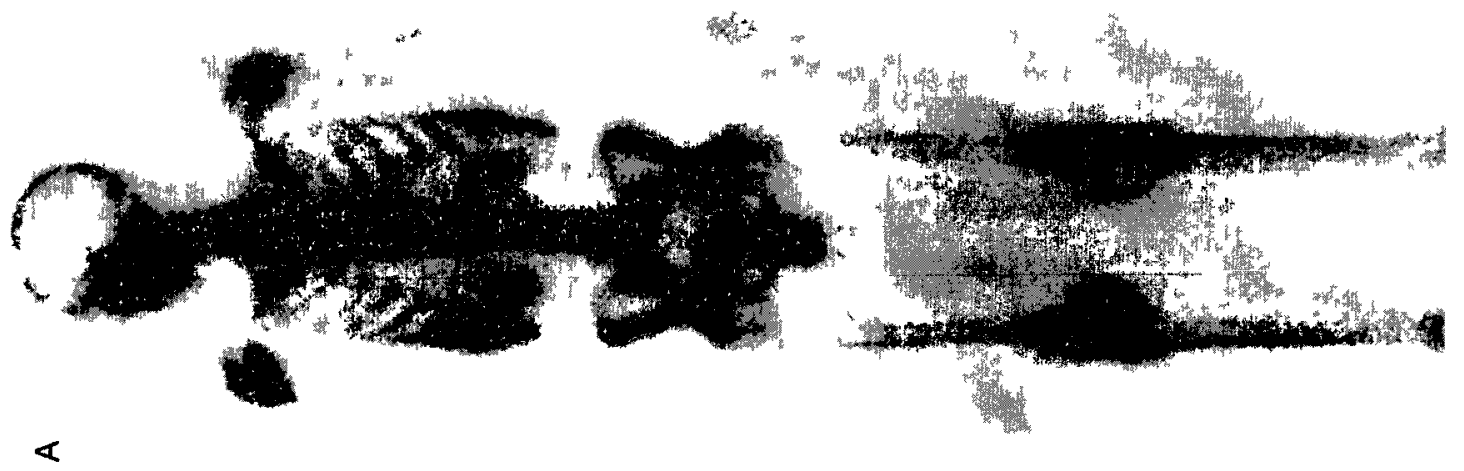

Figure 3 - Planar bone anterior and posterior images using ${ }^{99 \mathrm{~m}} \mathrm{Tc}$. Dark areas represent high concentrations of radioisotope-labeled tracer molecules ${ }^{3}$

\subsubsection{Tracer Molecules and Radioactive Isotopes}

Radiopharmaceuticals for diagnostic bone imaging using technetium are ${ }^{99 \mathrm{~m}} \mathrm{Tc}$ phosphates and phosphonates (20). Phosphonates accumulate in the mineral phase of bone and the rate of accumulation depends on two major factors: the blood flow to the bone and the extraction efficiency of the bone, which in turn depends on a number of biological variables (19). The radiopharmaceutical is administered intravenously, with

${ }^{3}$ This figure was published in Nuclear Medicine, $2^{\text {nd }}$ ed, Vol. 2, Podoloff, Donald A, Chapter 71 Malıgnant Bone Disease, Page 1183, Copyright Elsevier (2006), reproduced with permission 
$50 \%$ (average) of the tracer accumulating in the skeleton within one hour of the injection (19). The biological half-life of phosphonates is 26 hours (19). Cancerous bone lesions absorb an average of 17-18 times more ${ }^{99 \mathrm{~m}} \mathrm{Tc}-\mathrm{MDP}$ compared to healthy bone (21). The remaining tracer is rapidly cleared from the bloodstream by the kidneys, with an average of $9 \%$ of the injected dose accumulating in non-osseous tissue $(19 ; 21)$.

Planar bone imaging is also done with therapy radiopharmaceuticals, such as ${ }^{153} \mathrm{Sm}$-EDTMP (Ethylenediaminetetramethylene phosphonic acid) (22). EDTMP has similar bone-seeking properties and the same lesion-to-healthy absorption ratio as MDP, however, the ${ }^{153} \mathrm{Sm}$ isotope delivers a much higher radiation dose to the patient compared to ${ }^{99 \mathrm{~m}} \mathrm{Tc}(22)$. Relatively large quantities of ${ }^{153} \mathrm{Sm}$-EDTMP are administered to patients with metastatic cancer as palliative treatment (22). The primary radiation from ${ }^{153} \mathrm{Sm}$ is beta radiation (see Section 2.4.1.1), which damages and kills local tissue and, since bone lesions have a particularly high affinity for ${ }^{153} \mathrm{Sm}$-EDTMP, relieves some of the patient's pain. The secondary radiation from ${ }^{153} \mathrm{Sm}$ is gamma radiation, which is used for planar bone imaging in order to verify the effectiveness of the treatment (22). Often, images of pre-therapy ${ }^{99 \mathrm{~m}} \mathrm{Tc}$ scans are compared with $24-72$ hour post therapy ${ }^{153} \mathrm{Sm}$ scans to determine whether lesions in the ${ }^{99 \mathrm{~m}} \mathrm{Tc}$ image are shrinking or have been eliminated (23).

\subsubsection{Imaging Machine}

Modern SPECT imaging systems are multifunctional and capable of generating planar or computed tomography images using a variety of isotopes (14). Additionally, 
many SPECT systems have gantries for positioning patients and complex rotating and positioning equipment for the gamma camera head(s). These components will not be discussed here; only the general components of the gamma camera head and some of the data acquisition electronics will be described. For additional information on SPECT systems, Nuclear Medicine, $2^{\text {nd }}$ Edition is an excellent resource (11).

\subsubsection{Collimator}

The collimator is an essential component for providing spatial resolution in the gamma camera (24). There are several types of collimators for medical imaging, however, this section will be restricted to the most common type, the parallel-hole collimator, which is the type of collimator that was used for this experimental work. The collimator is a block of lead typically between two and five centimeters thick with a two-

dimensional symmetric array of circular, square or hexagonal holes (see Figure 4) (14). This lead grid is designed to block incident gamma rays that are not traveling on a nearperpendicular path relative to the detector face (24). However, the collimation is not perfect and some fraction of gammas from non-perpendicular origins will penetrate the collimator at the edges, blurring the image. Some smaller fraction will completely penetrate the collimator and create noise in the image (see Figure 4) (24).

Smaller holes and shorter distances between the collimator and the patient will result in better spatial resolution (14). However, there are practical limits to how close the gamma camera can be to a patient, and smaller collimator holes cause a decrease in 
system sensitivity (more gamma rays are blocked) because the spacing between holes must remain constant. Sensitivity of a collimator refers to the fraction of photons that reach the detector crystal face out of all the photons emitted by the source (assuming no photon scatter or absorption) and a typical value of sensitivity for a parallel-hole collimator is $\sim 0.03 \%$ (24). There is a tradeoff between the sensitivity and resolution which must be made for each imaging procedure (24).

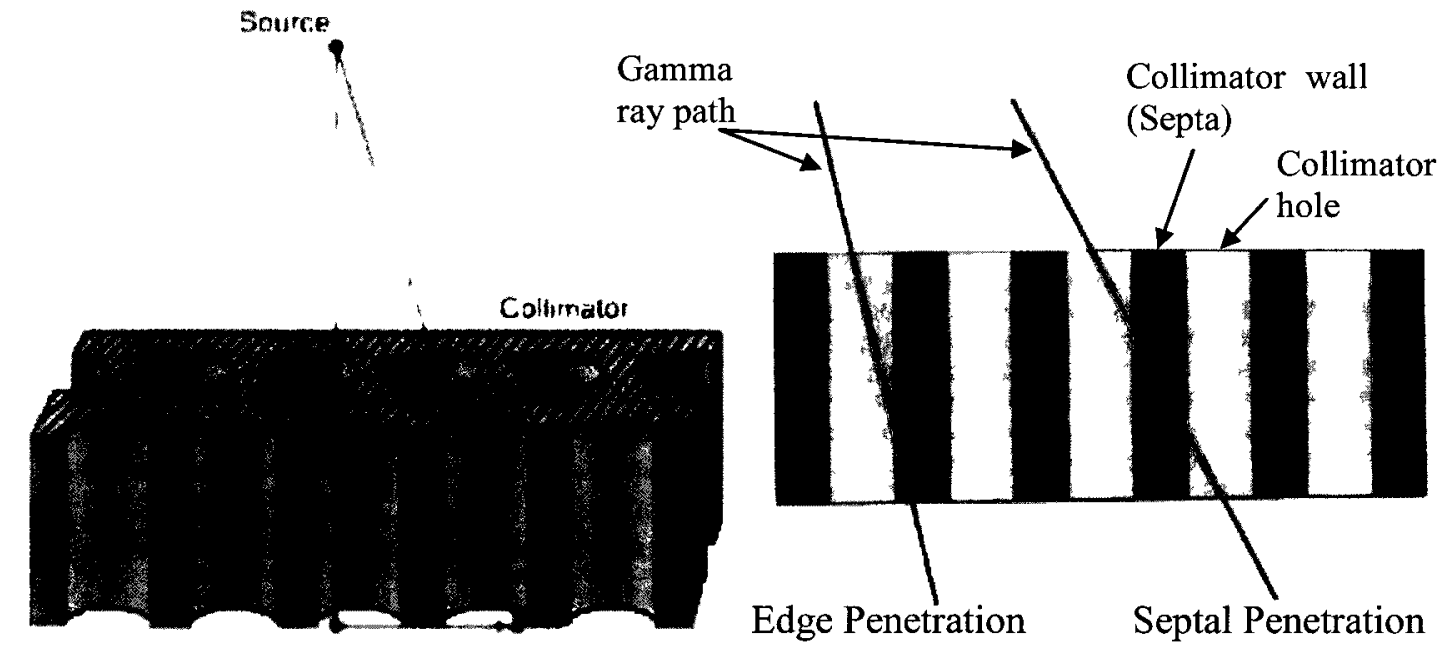

Figure 4 - Diagram of a parallel-hole collimator (left), illustrating the concept of a point source and its effective magnification by the collimator. Also shown is the concept of collimator penetration (right) which leads to image noise, blurring, and artifacts. ${ }^{4}$

${ }^{4}$ These figures were published in Nuclear Medicine, $2^{\text {nd }}$ ed., Vol. 1, Gunter, Donald L.; Halama, James R., Chapter 8: Gamma Camera Collimator Characteristics and Design, Page 109;118, Copyright Elsevier (2006), reproduced with permission. 


\subsubsection{Scintillation Crystal and Photomultiplier Tube Array}

The most common scintillation crystal used for SPECT imaging is the NaI(Tl) (Thallium-doped Sodium-Iodide) crystal (14). Most systems use a single crystal approximately $1 \mathrm{~cm}$ thick (though $1.6 \mathrm{~cm}$ thick crystals are available) and a circular or rectangular cross-section between 520 and 3200 square centimeters, depending on the application $(14 ; 25)$. When a gamma ray enters the $\mathrm{NaI}(\mathrm{Tl})$ crystal, it transfers energy to one or more electrons (26). The electron(s) subsequently scatters inside the crystal until all of its kinetic energy is dissipated, and in the process it produces many optical photons (27). A large fraction of the photons are collected by the photomultiplier tube (PMT) array and converted into electrons and amplified (28). The position of the original gamma ray event in the detector plane is calculated using the proportion of optical photons collected by each PMT (see Figure 5) (28). Statistical variations in the gamma ray conversion and photon amplification process introduce error into the photon positioning calculation, which degrades the spatial resolution of the imaging system (28). 


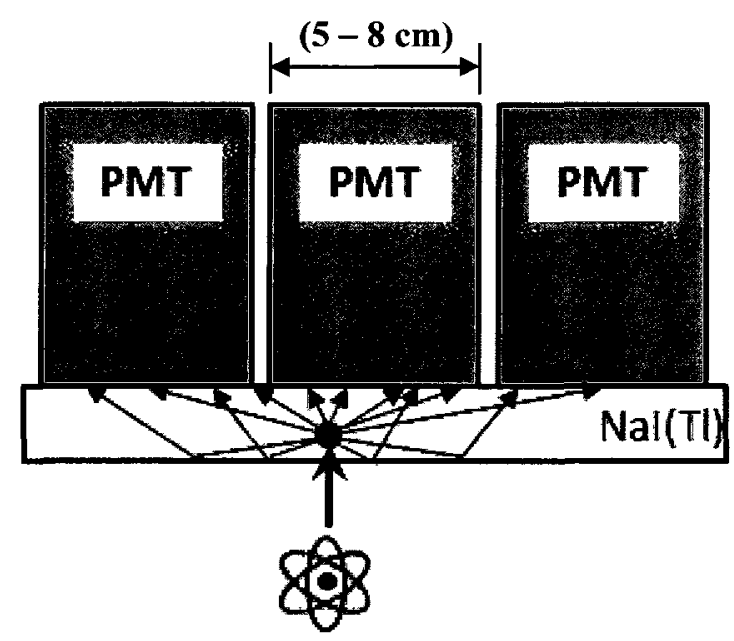

Figure 5 - PMT array on a $\mathrm{NaI}(\mathrm{Tl})$ crystal. A single gamma ray will generate a large number of optical photons in the crystal. As a result of the gamma ray interaction shown, the center PMT collects the most light, the left PMT collects the second highest amount of light, and the right PMT collects the least. The positioning circuit will calculate that the gamma ray was absorbed to the left of the central PMT.

\subsubsection{Pulse Height Analyzer and Gating Circuit}

The quantity of light released in the scintillation crystal is proportional to the amount of energy deposited by the gamma ray, with the maximum energy being deposited when the gamma ray is completely absorbed (28). Often, a gamma photon will not deposit all of its energy in the crystal. This is called a Compton event (see Section 2.4.2 for more information on Compton scattering). When this occurs, it is very difficult to distinguish between a photon arriving with only a portion of its initial energy (i.e., scattered from its initial path) and a photon that arrived with its full energy but escaped the detector before depositing all of it (14). To avoid recording erroneous spatial information, all partially detected photons are rejected (14). To reject these events, an 
acceptance energy window is established around the maximum gamma ray energy. A Pulse Height Analyzer (PHA) measures the amplitude of the photomultiplier tube voltage signal (14). If the voltage corresponds to a photon energy that is below or above the acceptance energy window, the event is discarded (the gate is closed) (14). If the voltage corresponds to a photon energy within the window, the gate is open and the event is recorded (14).

The width of the acceptance energy window is designed to accommodate the statistical variation of the signal energy (27). Statistical variations in the electron amplification process by the photomultiplier tubes cause the signal energy to vary with a Gaussian shape centered on the photon energy (see Figure 6) (27). The width of the acceptance energy window is designed to maximize the Signal to Noise Ratio (SNR) (27). As photon energy increases, the width of the full energy peak also increases, requiring a larger absolute acceptance energy window (27). 


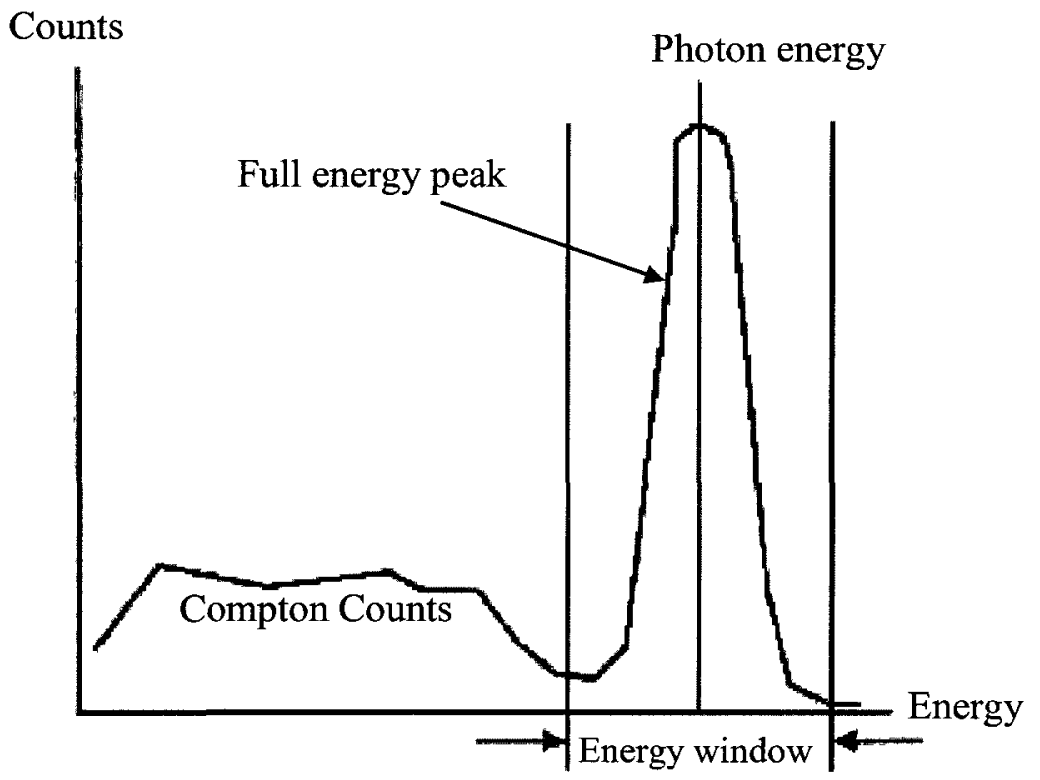

Figure 6 - Example of the spectrum and proportion of energies detected by a gamma camera from a mono-energetic source of photons. The energy window is centered on the full energy peak and its width is such that most Compton counts are rejected.

\subsubsection{Planar Imaging Procedure}

Planar bone imaging is normally done $2-5$ hours after the injection of ${ }^{99 \mathrm{~m}} \mathrm{Tc}-\mathrm{MDP}$ in order for the tracer to reach its maximum uptake in bone and a significant level of clearance from blood and non-osseous tissue (19). Occasionally, images may be taken between 6 and 24 hours post-injection in order to obtain a higher target to background signal ratio (19). The whole-body image is obtained by moving the patient beneath the stationary gamma camera (19). The duration of imaging varies relative to the amount of time that has passed since the injection, however, most scans are restricted to less than 45 minutes. Beyond that, patients become restless and their movement causes artifacts to appear in the image $(19 ; 29)$. 


\subsubsection{Image Quality}

In nuclear medicine imaging, there is a tradeoff between image quality and radiation dose to a patient (30). The goal is to keep the patient radiation dose as-low-asreasonably-achievable (ALARA principle) while still providing doctors with useful diagnostic information (16). To define 'usefulness', we must consider the purpose of a SPECT image, which is to provide a medical practitioner with sufficient information to make medical decisions with a reasonable level of confidence (30). A SPECT image can be objectively evaluated using a variety of metrics, such as the signal to noise ratio (SNR) or the spatial resolution $(30 ; 31)$. These mathematical tools for evaluating image quality are repeatable and the logic behind them is transparent, which makes them ideal for developing a unified image quality standard. However, SPECT images are ultimately evaluated by the eye and mind of a trained observer (the medical practitioner), which cannot be reliably modeled and replicated at present (32).

\subsubsection{Methods of Quantifying Image Quality}

An image can be considered useful (acceptable quality) if the features of the image pass some perception threshold of the observer (30). Research has shown that the ability to see something in an image depends on the experience and confidence of the observer (30). If the importance of the decision requires high confidence, then the threshold is raised and an image will have to possess higher than average contrast and SNR (30). An observer's confidence can be affected by several factors, including but not 
limited to experience, data from previous tests and analyses, and the consequences of the medical decision being made using the image data (30). However, there is a region of overlap where the majority of observers with a range of confidence levels will agree that an image is of usable quality.

There are methods to evaluate subjective image analysis. One of the more successful methods of image evaluation is called the receiver operating characteristic (ROC) curve analysis (30). This method employs multiple qualified observers who identify medical abnormalities in a series of images (30). The presence or absence of medical abnormalities in these images has been verified, so an observer's observations can be graded as correct or incorrect (30). This method is particularly useful in evaluating images that have been produced with different radiation exposure levels, in the interest of adhering to the ALARA principle (32). If a statistically significant number of observers have difficulty in correctly evaluating images that were produced with a common radiation exposure level, then the researchers can conclude that the particular exposure level is insufficient to generate useful medical images (32). Manual evaluation of a series of images for ROC analysis is a very time consuming process (32). Therefore, ROC analysis is typically reserved for when significant changes to procedures are suggested, such as those that would require an increase or decrease of radiation exposure to a patient (30).

SNR or resolution arguments are generally used when small changes in technology, such as a new reconstruction algorithm, filtering method, or collimator 
design are being proposed (30). The most useful form of SNR for evaluating image quality is based on the Rose Model. This model assumes that a signal is present as a sharp increase in photon flux on top of a uniform background (31). The signal and noise in the image are governed by the Poisson distribution. The formula for $\mathrm{SNR}_{\mathrm{Rose}}$ is (31):

$$
S N R_{\text {Rose }}=\frac{a_{T}\left(n_{S}\right)}{\sqrt{a_{T}\left(n_{S}+n_{b}\right)}}
$$

$n_{S}=$ Mean photon density of the signal (photons/unit area)

$n_{b}=$ Mean photon density of the background

$\mathrm{a}_{\mathbf{T}}=$ The area of the target feature

Rose had attempted to assign a threshold to the $\mathrm{SNR}_{\text {Rose }}$ that would allow for a simple quantitative method of evaluating image quality (31). Rose's research with a variety of observers and conditions suggested that a signal could be identified on a uniform background if the $\mathrm{SNR}_{\text {Rose }}$ was above $5 \pm 2$ (the range here is a symptom of the variability of the observers) (31).

\subsubsection{Methods of Enhancing Image Quality}

Background subtraction and filtering can be used to enhance image quality. Background subtraction can be used to improve the contrast in images with a uniform background. Filters used for medical image analysis can be linear, non-linear, stationary (i.e., filter function is independent of the local image structure), and adaptive (i.e., filter 
function varies with local image structure) (33). An example of a common stationary linear filter is a low-pass filter, which can be applied in the spatial or frequency domain to smooth out images (i.e., suppress high spatial frequencies) (33). Some of the more complex and successful filters are restoration filters, which use the Modulation Transfer Function (MTF) of the imaging system along with the noise power spectrum of the image to enhance the signal while simultaneously suppressing the background noise (33).

\subsubsection{Spatial Resolution and its Effect on Image Quality}

The two most significant factors affecting the resolution of a gamma camera system are the collimator (or extrinsic) resolution and the intrinsic resolution (14). Collimator resolution is the dominant factor in defining the gamma camera system resolution (14). The dimensions of the collimator features and the source-detector separation determine the collimator Point Spread Function (PSF) (14). The PSF describes the imaging system response to an isotropic point source (14). In the case of the collimator, a point source produces an approximately Gaussian distribution of signal intensity, illustrated in Figure 7 (14). The Full Width at Half Maximum (FWHM) of the Gaussian PSF is estimated by $R_{C}$, the collimator resolution, in equation 2 (14). 


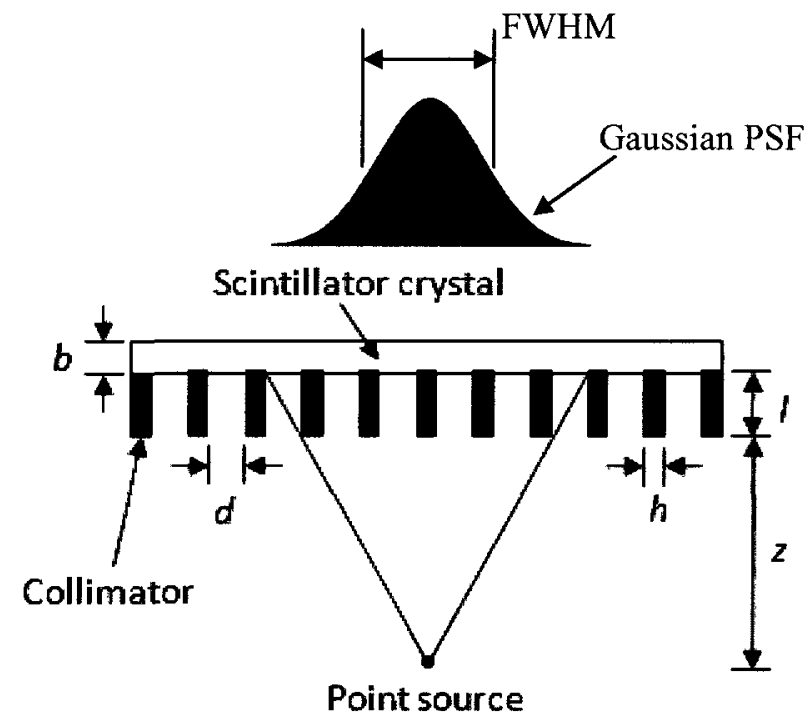

Figure 7 - Parallel hole collimator resolution (FWHM) for a point source. The resolution is a function of the collimator and crystal dimensions, as well as the source-todetector separation.

$$
\mathrm{FWHM} \cong R_{C}=\frac{d}{l}(l+b+|z|)
$$

The intrinsic resolution is a function of scintillator crystal and PMT effects (14). When a gamma ray Compton scatters in the crystal before being absorbed in a photoelectric event, the total gamma ray energy can be spread over multiple distinct regions of the crystal and the initial entry point of the gamma ray into the crystal is inaccurately calculated (14). Additionally, statistical variations in the crystal photon cascade and the amplification process of the PMTs introduce inaccuracy into the gamma ray positioning calculation (14).

Gamma ray sources with dimensions that are equal to or smaller than the detector system spatial resolution will appear to have reduced contrast in a gamma camera image (34). This effect is illustrated in Figure 8. For example, if it is important to identify all 
lesions over $10 \mathrm{~mm}$ in diameter, then a collimator with a spatial resolution smaller than $10 \mathrm{~mm}$ should be equipped. Low Energy High-Resolution (LEHR) and Low Energy AllPurpose (LEAP) collimators, used with ${ }^{99 \mathrm{~m}} \mathrm{Tc}$ (140 keV gamma rays), have spatial resolutions of approximately $6.4 \mathrm{~mm}$ and $8.3 \mathrm{~mm}$, respectively, with a point source $10 \mathrm{~cm}$ from the collimator face (25). A High Energy (HE) collimator, used with ${ }^{131} \mathrm{I}$ ( $364 \mathrm{keV}$ gamma rays), has a spatial resolution of $13.2 \mathrm{~mm}$ with a point source $10 \mathrm{~cm}$ from the collimator face (25). 

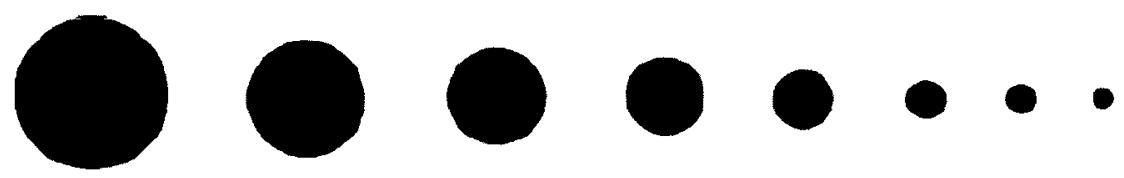

\section{$48 \mathrm{~mm} 36 \mathrm{~mm} 30 \mathrm{~mm} 24 \mathrm{~mm} 18 \mathrm{~mm} 12 \mathrm{~mm} 9 \mathrm{~mm} \mathrm{smm}$}
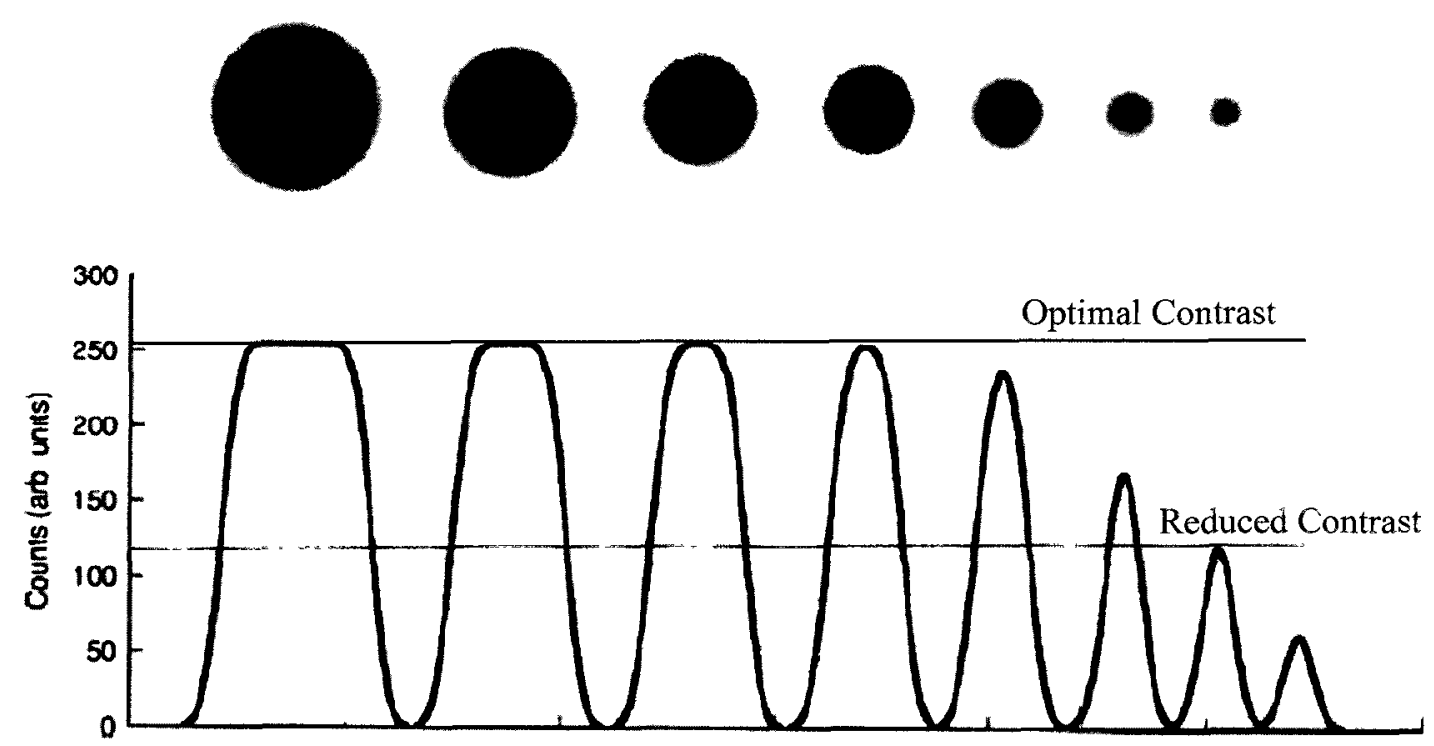

Figure 8 - An example of contrast reduction for lesions which are smaller than the spatial resolution of the detector system. The spatial resolution here is $12 \mathrm{~mm}$ FWHM. The top row lesions have diameters ranging from 6-48 $\mathrm{mm}$ and they each possess the same radiotracer concentration. The middle row is a simulation of the images that would be produced by scanning the top row. The bottom row shows the count profiles through the center of the images in the middle row. The integrated area under each count profile accurately reflects the total activity in the lesions. However, the maximum counts per unit length for lesions smaller than or equal to the system spatial resolution are reduced relative to lesions that are larger than the system spatial resolution. ${ }^{5}$

${ }^{5}$ This figure was published in Physics in Nuclear Medicine, $3^{\text {rd }}$ ed., Cherry, S.R.; Sorenson, J.A.; Phelps, M.E., Chapter 17: Single Photon Emission Computed Tomography, Page 318, Copyright Elsevier (2003), reproduced with permission. 


\subsubsection{Image Display}

The image matrix size is an important parameter affecting image quality. Experimental evidence has shown that the pixel width should be less than one third of the system FWHM in order to avoid loss of resolution (35). For example, if a system has a FWHM of $13.4 \mathrm{~mm}$, then the pixel width should be less than $4.5 \mathrm{~mm}$. A gamma camera with a Field of View (FOV) of $533 \mathrm{~mm}$ will have a pixel width of $8.3 \mathrm{~mm}$ for a $64 \times 64$ matrix, $4.2 \mathrm{~mm}$ for a $128 \times 128$ matrix, and $2.1 \mathrm{~mm}$ for a $256 \times 256$ matrix (35). In this example, a $128 \times 128$ matrix is ideal. A $256 \times 256$ matrix would also preserve the system spatial resolution, however, the total image counts are spread over more pixels, which will decrease the average pixel SNR.

\subsubsection{Radiation Dose}

Radiation dose is a measure of the amount of energy deposited in a medium (i.e., living tissue) by ionizing radiation and it can be quantified in three ways: absorbed, equivalent and effective (26). Absorbed dose is the energy absorbed per unit mass: the units are Grays (Gy), where 1 Gy equals 1 Joule per $\mathrm{kg}$ (26). The equivalent dose is the absorbed dose 'weighted' for the type of ionizing radiation and has units of Sieverts (Sv) (26). For example, fast (high-energy) neutrons have more potential to cause biological damage than photons. Table 1 contains weighting factors for various forms of ionizing radiation. One important feature of Table 1 is the different weighting factors for slow 
(low-energy) and fast (high energy) neutrons. Slow neutrons, which are used to activate the tracers for nSPECT, deliver a much lower equivalent dose than fast neutrons.

Table 1 - Radiation Weighting Factors (36)

\begin{tabular}{lc}
\hline Type of Radiation & Weighting Factor \\
\hline Photons (X-rays, Gamma rays) & 1 \\
Beta & 1 \\
Slow (thermal) neutrons & 2 \\
Fast neutrons & 10 \\
Alpha & 20 \\
\hline
\end{tabular}

Effective dose takes into account the energy absorbed by individual organs and their particular sensitivity to ionizing radiation (26). It also has units of Sieverts (Sv). Effective dose calculations are more complex and more accurate than the equivalent dose calculations, which treat all body parts equally (26).

\subsubsection{Dose to Patient from SPECT and Planar Bone Imaging}

The patient dose is due to the absorption of ionizing radiation emitted by the radioisotopes during their residence in the body (37). The effective dose depends on several factors: the initial administered activity, uptake efficiency, rate of drug metabolism, isotope physical half-life, tracer biological half-life, and the sensitivity of the target organ and surrounding organs (37).

The patient experiences the peak whole-body dose rate once the injected radioisotope solution reaches the blood stream shortly after the injection. The dose rate quickly declines within hours of the injection as the isotope that is not absorbed by tissue 
is cleaned from the blood stream and removed from the body through urination (20). The isotope that remains in the body is gradually removed according to the 26 hour biological half-life of the pharmaceutical, and the activity of the isotope drops with time due to its 6 hour physical half-life as well (37). The accepted range of administered activity for planar bone imaging in adults using ${ }^{99 \mathrm{~m}} \mathrm{Tc}$ is $740-1110 \mathrm{MBq}(20-30 \mathrm{mCi})$ (20). This yields an effective dose of $5.9-8.9 \mathrm{mSv}(20)$.

\subsubsection{Dose to Medical Practitioners}

Nuclear medicine physicians and technicians are permitted a maximum annual effective dose of $20 \mathrm{mSv}$, although this limit is rarely reached (38). Physicians tend to receive the lowest doses, because they typically only initiate the radiopharmaceutical injections and they do not need to be present throughout the whole injection time (38). Technicians receive the highest dose since they spend the most time in the vicinity of the patient, escorting them to and from the lab, positioning them in the imager and calibrating the gamma camera (38). Some technicians also prepare the ${ }^{99 m} \mathrm{Tc}$ drugs at the hospital, which increases their exposure, while other hospitals order unit-dose injections, which require little preparation (38). A study from 2002 at the Queen Mary Hospital in Hong Kong revealed that the average annual effective dose was $2.07 \pm 0.97 \mathrm{mSv}$ for radiographers (technicians), $0.29 \pm 0.21 \mathrm{mSv}$ for physicians, and $1.97 \pm 0.05 \mathrm{mSv}$ for nuclear medicine laboratory attendants (38). 


\subsection{The Physics of SPECT and nSPECT Imaging}

The following subsections describe the relevant physics of isotope decay and the interactions of high energy photons (X-rays and gamma rays) and neutrons with matter.

\subsubsection{Radioactive Decay}

Radioactive decay is the process of an unstable isotope releasing energy in order to reach a more stable nuclear configuration (26). The rate of decay of a group of radioactive atoms is called the "activity" and it is measured in units of Becquerels [Bq], which is equivalent to one decay per second $\left[\mathrm{s}^{-1}\right](26)$. Radioactive decay is a random process, so parameters such as "activity" describe the average behavior of a group of radioactive particles (26). An important parameter used to describe radioactive decay is the physical half-life, $t_{1 / 2}$. The physical half-life is the amount of time required for half of a quantity of a radioactive isotope to decay (26). The energy that is released during decay can have different forms depending on the nuclear configuration of the decaying isotope (26). The two decay types that are most relevant to this thesis are the beta minus $\left(\beta^{-}\right)$ decay and the isomeric transition.

\subsubsection{Beta Decay}

In beta minus decay, a neutron in the nucleus emits an electron and becomes a proton (26). When this occurs, the atomic number of the decaying atom increases by one and the mass number remains constant (for example, ${ }^{153} \mathrm{Sm}$ beta decays into ${ }^{153} \mathrm{Eu}$ ). A 
neutrino is also released (26). The total energy released in the beta decay is a function of the pre-decay and post-decay atomic masses, and this total energy is shared between the beta particle and the neutrino (26). The neutrino is relevant to nuclear medicine only because it carries away some fraction of the decay energy and does not transfer any energy to the patient (26). After the beta decay, the nucleus is often left in an excited energy state and this energy can be released as one or more gamma rays (as is the case with ${ }^{153} \mathrm{Sm}$ and $\left.{ }^{131} \mathrm{I}\right)(26)$. In some cases, the excited energy state is sufficiently stable to have a physical half-life ranging from seconds to days, in which case the excited nucleus is referred to as an isomer (26).

The beta particle (or electron) can have a range of kinetic energy, which can be described by a continuous probability distribution (26). Because the beta particle is a charged particle, it transfers its kinetic energy to the surrounding matter in short distance; in the human body, the range of typical beta particles is expressed in terms of millimeters (26). This property of beta particles is the reason why isotopes like ${ }^{153} \mathrm{Sm}$ and ${ }^{131} \mathrm{I}$ are useful for some radiation therapy treatments, where the objective is to destroy malignant cells (16). Some of the gamma ray(s) that are often released by the excited nucleus following a beta emission can be used for nuclear medicine imaging, allowing physicians to monitor the distribution of the therapy radioisotopes in the patient (16).

Conversely, diagnostic imaging requires that the radiation dose to the patient be as low as reasonably achievable (ALARA) (16). Therefore, isotopes which emit beta particles and gamma rays are not desirable for diagnostic imaging (16). Isotopes which 
emit gamma rays without any high-energy electrons or other charged particles, such as ${ }^{99 \mathrm{~m}} \mathrm{Tc}$, are preferable (16).

\subsubsection{Isomeric Transition}

After a beta particle emission, it is possible that the nucleus is left in an excited state; that is, it has not yet completed its transition to the new isotope (26). Usually, the nucleus is in this excited state for nanoseconds and releases a gamma ray or an electron to carry away the excess energy (26). For a few isotopes, this excited or meta-stable state can last from seconds to days (26). An example is ${ }^{99 m} \mathrm{Tc}$, where the ' $\mathrm{m}$ ' indicates that it is in a meta-stable state. ${ }^{99 \mathrm{~m}} \mathrm{Tc}$ is an intermediate state between ${ }^{99} \mathrm{Mo}$ and ${ }^{99} \mathrm{Tc}$ with a physical half-life of 6 hours, which makes it useful for medical imaging (26). The relatively long metastable physical half-life provides a sufficient amount of time to

separate the gamma-emitting ${ }^{99 \mathrm{~m}} \mathrm{Tc}$ from the beta-emitting ${ }^{99} \mathrm{Mo}$ and use ${ }^{99 \mathrm{~m}} \mathrm{Tc}$ for imaging (26).

\subsubsection{Statistics of Radioactive Decay}

The random process of radioactive decay is described by the Poisson distribution (14). Therefore, the mean number of radioactive decays within a given amount of time is also equal to the variance. The mean number of decays, $\Delta N$, is (14):

$$
\Delta N=\lambda N \Delta t
$$


where $N$ is the number of radioactive atoms, $\Delta t$ is the time interval, and $\lambda$ is the transformation constant for the radioactive isotope and is related to the physical half-life as $\lambda=\ln (2) / t_{1 / 2}$. The activity (the number of decays per unit time) is then $A=\Delta N / \Delta t=$ $\lambda N(14)$.

\subsubsection{Gamma Radiation Interaction with Matter}

Gamma radiation is the name of a class of high-energy electromagnetic radiation (i.e., photons) that originates in the nucleus of an atom. The energy of gamma rays is usually expressed in units of electron volts $(\mathrm{eV})$. An electron volt is equal to the amount of kinetic energy gained by an electron as it is accelerated through an electric potential difference of $1 \mathrm{~V}\left(1 \mathrm{eV}=1.6 \times 10^{-19} \mathrm{~J}\right)(26)$.

There are four types of photon interactions with matter that comprise the linear attenuation coefficient for gamma rays in matter in general: these are coherent scattering, incoherent (Compton) scattering, photoelectric absorption and pair production (26). For coherent scattering, the electrons in an atom redirect the photon without absorbing any of its energy (26). Compton scatter, however, is an interaction between the photon and a single electron and some amount of energy less than the total energy of the photon is transferred to the electron as kinetic energy (26). In a photoelectric interaction, all of the photon energy is absorbed by an electron and converted to kinetic energy, minus the electron binding energy (26). Pair production is unique in that it is an interaction with the nucleus of an atom (26). When a photon passes near the nucleus of an atom the strong 
field of the nucleus converts the photon into an electron-positron pair; the reaction has a threshold energy of $1022 \mathrm{keV}$ since the mass of an electron/positron is equivalent to 511 $\mathrm{keV}$ (26). All of these interactions contribute to the total photon linear attenuation coefficient of a material, as illustrated in Figure 9 (26).
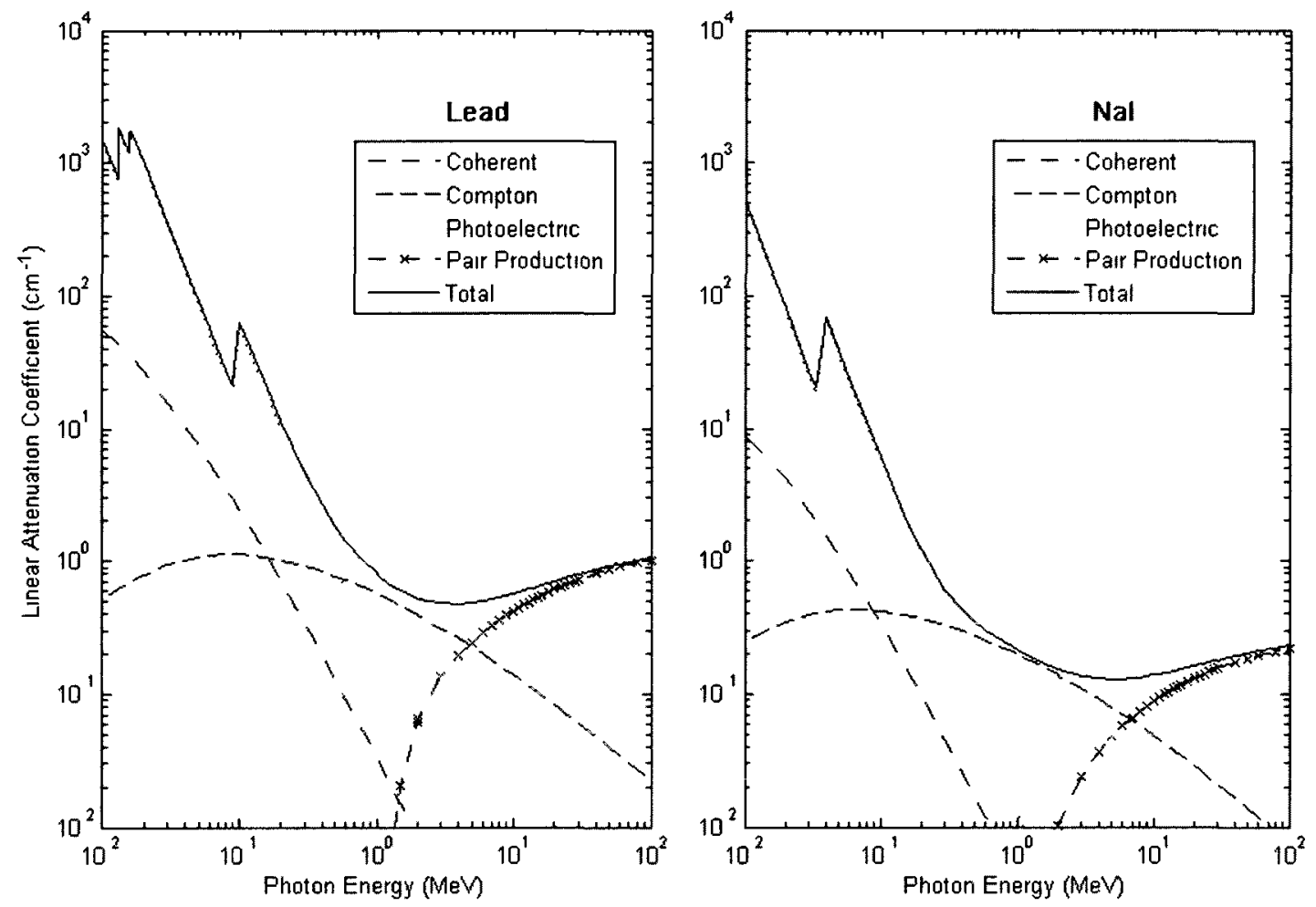

Figure 9 - Photon linear attenuation coefficient versus photon energy for NaI and Lead. Each plot shows the linear attenuation coefficient for each type of interaction, as well the total linear attenuation coefficient. The data used to generate these plots is from reference (39).

The linear attenuation coefficient is used to describe the average number of photons that will be scattered and/or absorbed when passing through a material (26). The 
equation describing the fraction of photons removed from a beam of photons passing through a material is (13):

$$
\text { Attenuation fraction }=1-e^{-\mu x}
$$

where $\mu$ is the total linear attenuation coefficient (units of length ${ }^{-1}$ ) of the material and $x$ is the thickness of the material (units of length).

For $\mathrm{NaI}$ (the gamma camera crystal material) and gamma energies above 300 $\mathrm{keV}$, Compton scattering is the dominant photon interaction, whereas below $300 \mathrm{keV}$ photoelectric interactions dominate. For the NaI gamma camera and the lead collimator, photoelectric events are preferred since all of the photon energy is transferred to an electron in a single event (28). When one or more Compton events occur, the photon has a chance to escape the material before being consumed in a photoelectric event (28). This is why it is generally undesirable to image with higher-energy photons. For lead, photoelectric events remain dominant above $500 \mathrm{keV}$, which, in addition to its high density, is why it is so commonly used as a collimator and shielding material (26).

In the past, when ${ }^{99 \mathrm{~m}} \mathrm{Tc}$ was not as available as it is today, gamma cameras were optimized for higher energy photons from isotopes like ${ }^{131} \mathrm{I}(364 \mathrm{keV})$. The high-energy optimization required thicker crystals ( $\sim 12.7 \mathrm{~mm}$ thick), which are more likely to absorb photons after a Compton interaction (28). Modern crystals optimized for ${ }^{99 \mathrm{~m}} \mathrm{Tc}$ photons $(140 \mathrm{keV})$ are thinner $(\sim 9.4 \mathrm{~mm}$ thick$)$ and also less expensive. Moving to the $9.4 \mathrm{~mm}$ thick crystals sacrificed very little efficiency at $140 \mathrm{keV}$; from $90 \%$ complete photon 
absorption in the $12.7 \mathrm{~mm}$ crystals down to $80 \%$ complete photon absorption for $9.4 \mathrm{~mm}$ thick crystals (28). At higher energies around $364 \mathrm{keV}$, however, moving to the $9.4 \mathrm{~mm}$ crystals caused a significant drop in efficiency; from $30 \%$ complete photon absorption for $12.7 \mathrm{~mm}$ crystals down to $20 \%$ complete photon absorption for $9.4 \mathrm{~mm}$ crystals (28).

\subsubsection{Prompt Gamma Neutron Activation Analysis (PGNAA)}

PGNAA is the central idea for the nSPECT method. Neutron radiation for PGNAA is produced from fission (such as ${ }^{235} \mathrm{U}$ in a nuclear reactor), from fusion (such as a ${ }^{2} \mathrm{H}+{ }^{2} \mathrm{H}$ reaction in an accelerator), or from radioactive decay (13). When an atom is bombarded with neutron radiation, a neutron may come into contact with the nucleus and the neutron will be scattered or absorbed (13). If the neutron is absorbed by the nucleus, the nucleus may become unstable after transitioning to a new isotope and it will release energy, such as in the form of a photon (gamma ray), in order to achieve a stable energy state (illustrated in Figure 10) (13). If this process occurs in a time interval smaller than a few microseconds, it is considered Prompt Gamma Neutron Activation (PGNA) (13).

Each isotope has a unique probability of radiative neutron capture (called a radiative thermal neutron capture cross-section) and a unique set of emitted gamma photon energies (13). Isotopes in which radiative neutron capture is more likely to occur have a high cross-section (13). Neutron capture cross-sections are quoted for a single nucleus and are measured in barns $\left(1 \mathrm{barn}=10^{-24} \mathrm{~cm}^{2}\right)$ and can vary by several orders of 
magnitude between isotopes and elements (13). The equilibrium activity of an isotope in a neutron radiation field is given by the formula (13):

$$
A=\sigma \varphi N
$$

where $\sigma$ is the neutron capture cross-section of the isotope in $\mathrm{cm}^{2}, \varphi$ is the neutron flux in (n $\mathrm{cm}^{-2} \mathrm{~s}^{-1}$ ), and $N$ is the number of atoms with cross-section $\sigma$ exposed to the neutron flux $\varphi$.

Data for a few relevant isotopes are shown in Table 2. Of the isotopes listed, ${ }^{149} \mathrm{Sm}$ and ${ }^{157} \mathrm{Gd}$ are the most likely to be activated if exposed to a thermal neutron flux. The most probable photon emissions of ${ }^{157} \mathrm{Gd}$ and ${ }^{149} \mathrm{Sm}$ are within the usable range for SPECT imaging. The primary constituents of the human body (oxygen, carbon and hydrogen) are also shown in Table 2. Oxygen and carbon have negligible PGNA crosssections. Hydrogen, however, has a much higher cross-section and although its crosssection is still very small compared to the potential nSPECT tracer isotopes ${ }^{149} \mathrm{Sm}$ and ${ }^{157} \mathrm{Gd}$, hydrogen is the most abundant atom in the human body (26). Equation (5) shows that activity is a function of the product of $\sigma$ and $N$, so an abundance of low $\sigma$ water can have comparable activity to trace amounts of high $\sigma^{157} \mathrm{Gd}$ or ${ }^{149} \mathrm{Sm}$. 
Target Nucleus

Incident
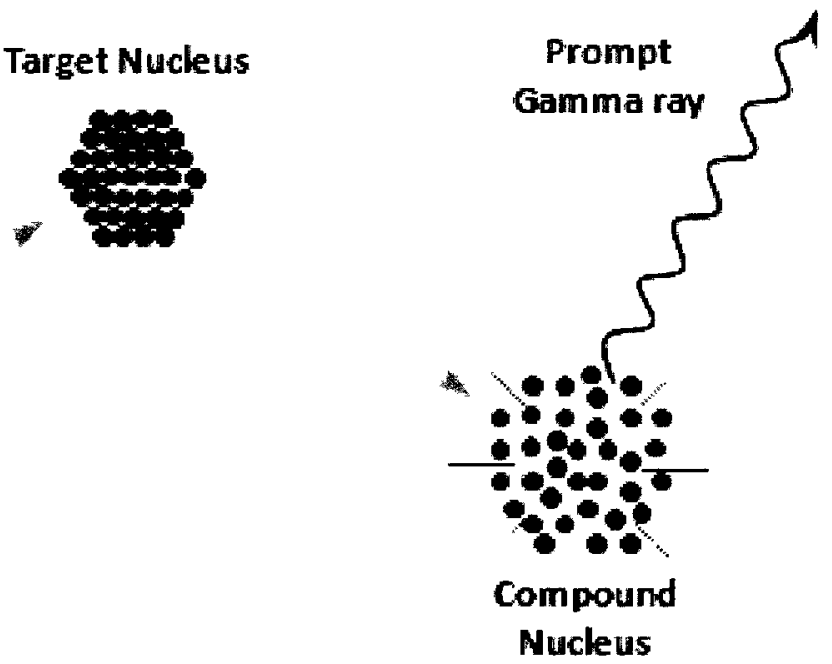

Figure 10-Illustrating the process of prompt gamma neutron activation

Table 2 - Some relevant isotopes and their most significant neutron capture crosssections and corresponding gamma-ray emission energies. The natural abundance of an isotope is the average percentage of that isotope that is present in an elemental sample found in nature (for example, natural oxygen contains $\left.{ }^{16} \mathrm{O},{ }^{17} \mathrm{O},{ }^{18} \mathrm{O}\right) .(40 ; 13)$

\begin{tabular}{|ccccc|}
\hline Isotope & $\begin{array}{c}\text { Natural } \\
\text { Abundance }\end{array}$ & $\begin{array}{c}\text { Maxwellian Avg. PGNA } \\
\text { Cross-section at 298 K } \\
\text { (barns or 10 } \mathbf{c m}^{-24} \text { ) }\end{array}$ & $\begin{array}{c}\text { Emission } \\
\text { Probability }\end{array}$ & $\begin{array}{c}\text { Photon } \\
\text { Energy }\end{array}$ \\
\hline${ }^{157} \mathbf{G d}$ & $15.65 \%$ & 127000 & $\begin{array}{c}10 \% \\
18 \%\end{array}$ & $\begin{array}{c}80 \mathrm{keV} \\
182 \mathrm{keV}\end{array}$ \\
\hline${ }^{149} \mathbf{S m}$ & $13.82 \%$ & 57000 & $86 \%$ & $334 \mathrm{keV}$ \\
& & $1.21 \times 10^{-3}$ & $52 \%$ & $439 \mathrm{keV}$ \\
\hline${ }^{16} \mathbf{O}$ & $99.76 \%$ & $2.05 \times 10^{-2}$ & $36 \%$ & $870 \mathrm{keV}$ \\
\hline${ }^{12} \mathbf{C}$ & $98.89 \%$ & 0.365 & $100 \%$ & $2223 \mathrm{keV}$ \\
\hline${ }^{\mathbf{1}} \mathbf{H}$ & $99.985 \%$ & & & \\
\hline
\end{tabular}




\section{5 nSPECT for Bone Imaging}

nSPECT is a novel method of applying in-vivo PGNAA with a gamma camera imaging system to determine the spatial distribution and density of the tracer $\operatorname{drug}(\mathrm{s})$, which provides information on the biological process of interest. The drug(s) containing the stable target isotope(s) would be administered to a patient, who would then be placed in a modified SPECT imaging system (illustrated in Figure 11). The neutron source would be turned on, exposing the patient to low-energy neutrons, thus activating the tracer, and the activated isotopes would emit gamma rays that would be imaged. Once imaging is complete, the neutron source would be turned off and the tracer would cease to be radioactive. nSPECT could offer many advantages over modern SPECT techniques, as described in the introduction (see page 4).

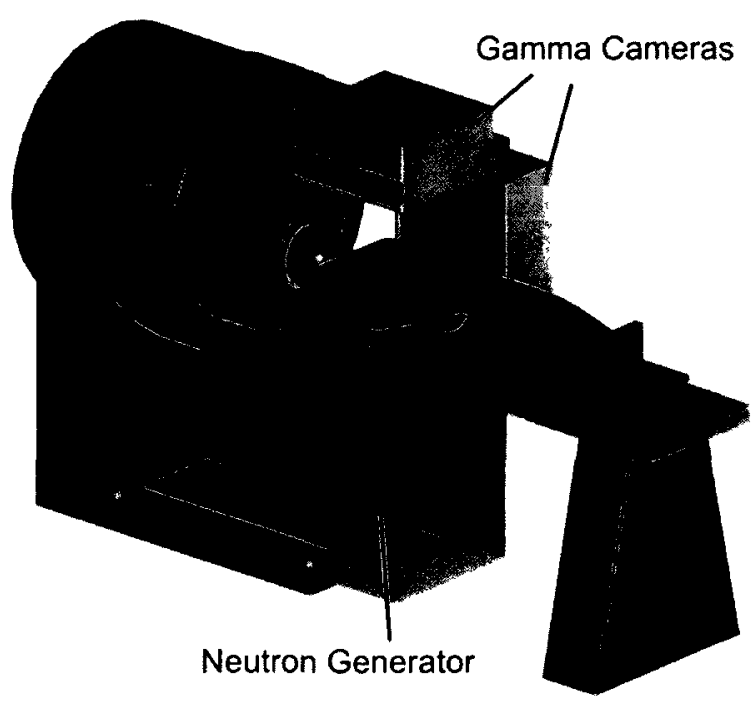

Figure 11 - An illustration of the nSPECT system concept, which consists of a modern SPECT system coupled with an electric, accelerator-based neutron generator. The neutron generator may have a fixed location or it may be mobile, like the gamma cameras. Neutron moderator and radiation shielding are not shown. 
For this method of neutron activated imaging to be successful, the tracer isotope should have a large neutron absorption cross-section and the resulting gamma ray emission should ideally be between 100 and $250 \mathrm{keV}$ (the design point of modern SPECT detectors), though SPECT radioisotopes with gamma ray energies between 70 and 364 $\mathrm{keV}$ are used successfully (16). The isotope must also be chemically compatible with a pharmaceutical delivery system.

${ }^{149} \mathrm{Sm}$ is ideal for nSPECT bone imaging. It has a primary photon emission (334 $\mathrm{keV}$ ) within the same energy range of current SPECT radioisotope tracers and a secondary photon emission (439 keV) that may also be usable for imaging (13). Both of these ${ }^{149} \mathrm{Sm}$ photon energies are above the optimal energy range of the $1 \mathrm{~cm}$ thick crystals in most modern SPECT detectors, so there would be a significant efficiency gain if a detector with the less common $1.6 \mathrm{~cm}$ crystals were used (see Section 2.4 .2$)(28) .{ }^{149} \mathrm{Sm}$ also has a very large thermal neutron cross-section of 57,000 b (Table 2, pg. 41). Additionally, its chemical compatibility with a bone-seeking molecule, EDTMP, is already well-known from the ${ }^{153} \mathrm{Sm}$-EDTMP radiation therapy drug (21). Ample data are available regarding the pharmacokinetics of samarium-labeled EDTMP $(21 ; 23)$.

\subsubsection{Neutrons in Medicine}

nSPECT would not be the first occasion where neutron radiation was used in medicine. In Boron Neutron Capture Therapy (BNCT), the ${ }^{10} \mathrm{~B}$ isotope is attached to a carrier which seeks out malignant tissue (41). ${ }^{10} \mathrm{~B}$ has a large $(3600 \mathrm{~b})$ neutron absorption 
alpha emission cross-section; an alpha particle is a helium nucleus with a charge of +2 and it deposits all of its kinetic energy from the reaction in a very short distance (typically measured on the scale of a cell-width) (26;13). After the boron drug has been administered to the patient, the target area in the patient is exposed to a high neutron flux (between 0.20 and $4.3 \times 10^{9} \mathrm{n} \mathrm{cm}^{-2} \mathrm{~s}^{-1}$ ) for up to 30 minutes and the resulting alpha particles kill any tissue containing boron $(41 ; 42)$. Clinical trials with BNCT are being conducted in several countries at facilities that operate nuclear research reactors (41). The research reactors are necessary to achieve the high neutron fluxes required for BNCT (41). These high fluxes are not required for nSPECT because it is a diagnostic method, not a radiation therapy method like BNCT, and so relatively small electric neutron sources should provide a sufficient neutron flux for nSPECT.

A group from the Medical Physics and Applied Radiation Sciences Department at McMaster University has done research for in-vivo PGNAA (43; 44). Their technique uses a neutron source to irradiate a particular body part or organ and activate the tissue and its contents (43). Using a high-purity germanium (HPGe) detector, they detect photons originating from trace elements such as cadmium, mercury, or other undesirable contaminants and quantify the amounts of these materials that are present (43). This is not an imaging technique; they are concerned with how much material is present, not the spatial distribution of that material. 


\subsubsection{EDTMP Chemical Dose Limit}

In addition to minimizing the radiation dose to a patient to within safe limits for nSPECT, it is important that the chemical dose from the pharmaceutical be within safe limits as well. In addition to its use as a bone seeking compound in medical applications, EDTMP is found in various household detergents (45). Daily oral consumption as high as $100 \mathrm{mg} / \mathrm{kg} /$ day for 13 weeks in rats demonstrated no adverse affects (46). At a consumption of $500 \mathrm{mg} / \mathrm{kg} / \mathrm{day}$, rats showed statistically significant haematological indications of mild anemia (due to iron deficiency) from week 6-13 (46). It is suspected that the anemia resulted from the chelation of iron by EDTMP in the digestive system (46). Four weeks after the $500 \mathrm{mg} / \mathrm{kg} /$ day EDTMP consumption ended, the rats fully recovered from the mild anemia without any intervention in their normal diets (i.e., no nutritional supplements, such as additional iron, were provided) (46). Bone uptake (3.2\%) accounted for half of total tissue uptake of $6.3 \%$ of daily consumption (46). EDTMP has a biological half-life of approximately 520 hours in bone (22), but it is cleared from nonosseous tissue within a few hours (21).

The ${ }^{149}$ Sm-EDTMP complex can be synthesized with a near $1: 1$ molar ratio with a very high $(99 \%)$ purity using methods that are currently used to produce the ${ }^{153} \mathrm{Sm}$ EDTMP radiopharmaceutical (47). The total quantity of $\mathrm{Sm}$ deliverable to an average human skeleton was assessed for daily oral dosing up to 21 days (see Figure 12, calculations are found in Appendix A). Both $100 \mathrm{mg} / \mathrm{kg} /$ day and $500 \mathrm{mg} / \mathrm{kg} /$ day EDTMP doses were considered for a $70 \mathrm{~kg}$ person. Since the regimen is only a maximum of three 
weeks in length, the mild anemia experienced by rats consuming $500 \mathrm{mg} / \mathrm{kg} /$ day in weeks 6-13 should be avoided.

It is also possible to administer ${ }^{149} \mathrm{Sm}$-EDTMP intravenously, as is typically done for ${ }^{153} \mathrm{Sm}$-EDTMP and many SPECT radioisotope tracers (48). In this case, an average of $50 \%$ of the tracer is accumulated in bone, while the rest is rapidly cleared from the blood by the kidneys (22). The limit to the quantity of ${ }^{149} \mathrm{Sm}$-EDTMP that can be administered intravenously is unknown; there is concern that excessive quantities injected over a short period of time could overload and harm the kidneys, particularly if patients have a preexisting kidney condition (49).

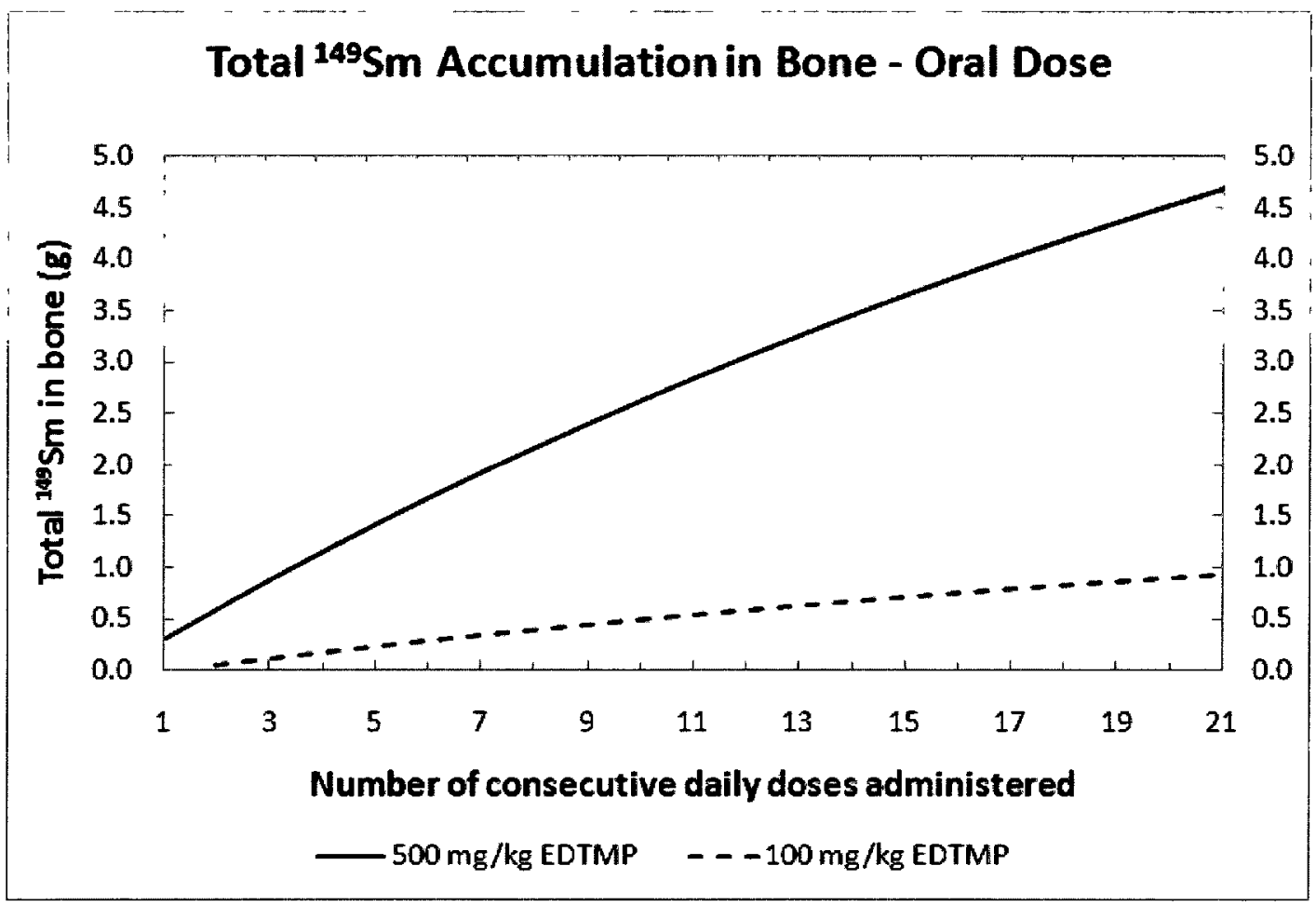

Figure 12 - A plot of the total ${ }^{149} \mathrm{Sm}$ mass deposited on the skeleton from a daily oral dosing regimen of ${ }^{149} \mathrm{Sm}$-EDTMP. This takes into account the bioavailability of EDTMP as well as its natural rate of removal from bone (biological halflife). The patient is assumed to have a mass of $70 \mathrm{~kg}$. 


\subsubsection{Potential Technical Obstacles}

The most significant uncertainties about nSPECT are:

- The number of gamma counts required to create an acceptable image

- The patient radiation dose (in $\mathrm{mSv}$ )

- The corresponding tracer dose (mg of ${ }^{149} \mathrm{Sm}$-EDTMP)

Typically, a SPECT image acquisition time is dictated by the number of gamma counts detected by the camera (19). For a particular procedure, the approximate number of counts required to create an acceptable image is known from experience. The radiopharmaceutical dose is adjusted for each patient to ensure that the gamma count rate is sufficient to achieve the required total counts in less than 45 minutes, which is the maximum amount of time that a patient can be expected to lie still (29).

For nSPECT bone imaging, one might propose that the count requirement should be identical to the requirement for ${ }^{99 \mathrm{~m}} \mathrm{Tc}-\mathrm{MDP}$ bone imaging, however, this may not be the case due to some important differences between the two methods. With nSPECT, there will be a higher background count-rate due to Compton-scattered photons from hydrogen PGNA, which will degrade image contrast and quality, thus requiring more total signal counts than ${ }^{99 \mathrm{~m}} \mathrm{Tc}-\mathrm{MDP}$ to achieve equivalent image quality. Additionally, for

${ }^{149} \mathrm{Sm}$, the photon energy is higher than ${ }^{99 \mathrm{~m}} \mathrm{Tc}$, which means that the resolution of the imaging system will be reduced (see Section 2.3.2.1 and 2.3.4.3). Therefore, lesions that are less than $2 \mathrm{~cm}$ in diameter which are visible with ${ }^{99 \mathrm{~m}} \mathrm{Tc}$ will require more counts to 
achieve the same result with ${ }^{149} \mathrm{Sm}$ PGNA. In the case of highly localized imaging, such as imaging an extremity, for example, the neutron beam and activity from ${ }^{149} \mathrm{Sm}$ could be limited to an area of interest. This would result in a lower total number of required counts compared to ${ }^{99 \mathrm{~m}} \mathrm{Tc}-\mathrm{MDP}$, which has counts coming from areas outside of the region of interest.

For in-vivo PGNAA, neutrons are typically the primary contributor to patient dose (43). For accurate neutron dosimetry, experimental and/or simulation data are required. For nSPECT, it should be possible to adjust the neutron flux (and, consequently, the radiation dose) to maintain a constant target activity if the quantity of tracer isotopes is also adjusted to compensate. This relationship is shown in equation 5 in Section 2.4.3. For example, if we reduce the neutron flux, $\varphi$, (and therefore the patient dose) by a factor of 2 and increase the quantity of tracer, $N$, in the target by a factor 2 , then the activity, $A$, remains constant. However, in addition to the patient safety concerns of increasing the pharmaceutical tracer dose (see Section 2.5.2), there is also an issue of neutron flux depression in the target, which is the point where the neutron absorption rate in a material becomes sufficiently high that the neutron flux in the surrounding material is significantly reduced (13). Substantial neutron flux depression could result in some lesions being 'hidden' in a patient, since the reduced neutron flux would result in reduced activity. Neutron flux depression is primarily a concern regarding deep-lying lesions, where neutron scattering and absorption in the intermediate tissue will reduce the activity in deeper tissue (13). 


\section{CHAPTER 3: EXPERIMENTS}

Two experiments were conducted. The first experiment was conducted by James Gräfe and David Chettle of McMaster University in collaboration with the author. The objective of that experiment was to estimate the dose rate and neutron flux attenuation characteristics of a Sm target. These data were required for planning the second experiment, performed by the author, the results of which were used to estimate the image quality for ${ }^{149} \mathrm{Sm}-\mathrm{EDTMP} \mathrm{nSPECT}$.

\subsection{Summary of the Preliminary Experiment by McMaster University}

Members of the McMaster University Medical Physics and Applied Radiation Sciences Department are experienced with in-vivo PGNAA (43; 44). The researchers there have worked at identifying toxic, large neutron cross-section elements like cadmium and mercury inside kidneys using a detector without spatial resolution (43). They have conducted experiments using both the McMaster Nuclear Reactor (MNR) and a small Plutonium-Beryllium $(\mathrm{PuBe})$ radioisotope source to irradiate a phantom with neutrons (43). They have then simulated those experiments using the Monte Carlo software MCNP and used the experimental data to validate the model (43). The simulations allow them to calculate the neutron flux and, therefore, the radiation dose rate to the phantom (43). MCNP is a general-purpose Monte Carlo N-Particle code developed by the Los Alamos National Laboratory for modeling the interaction of radiation with everything (50). 
The author collaborated with the team at McMaster University to plan an experiment that was similar to their past work with heavy metals. Natural abundance samarium (see Table 2, pg.41) was used as the activation target instead of the various heavy metal targets used in previous experiments. The accuracy of the model produced by the McMaster University team had already been established and their expertise with dosimetry calculations yielded a reliable neutron flux-dose rate relationship for samarium in a short period of time.

\subsubsection{Experiment Description}

Figure 13 is a simplified diagram of the experimental setup. The McMaster University team conducted the experiment with their PuBe radioisotope neutron source. The source emits approximately $4 \times 10^{7}$ neutrons per second isotropically and it is collimated and moderated with beryllium to produce a wide neutron beam composed primarily of thermal neutrons (43). A $125 \mathrm{~mL}$ polyethylene bottle was filled with a natural abundance samarium solution and placed inside a water tank (to simulate a target inside a human body) within the neutron beam. To detect the gamma ray emissions from the ${ }^{149} \mathrm{Sm}$ PGNA, a single gamma-ray detector is placed near the target. Several different concentrations of natural abundance Sm solution were used and each measurement was integrated over 2000 seconds. Each experimental measurement was modeled with an MCNP simulation. 


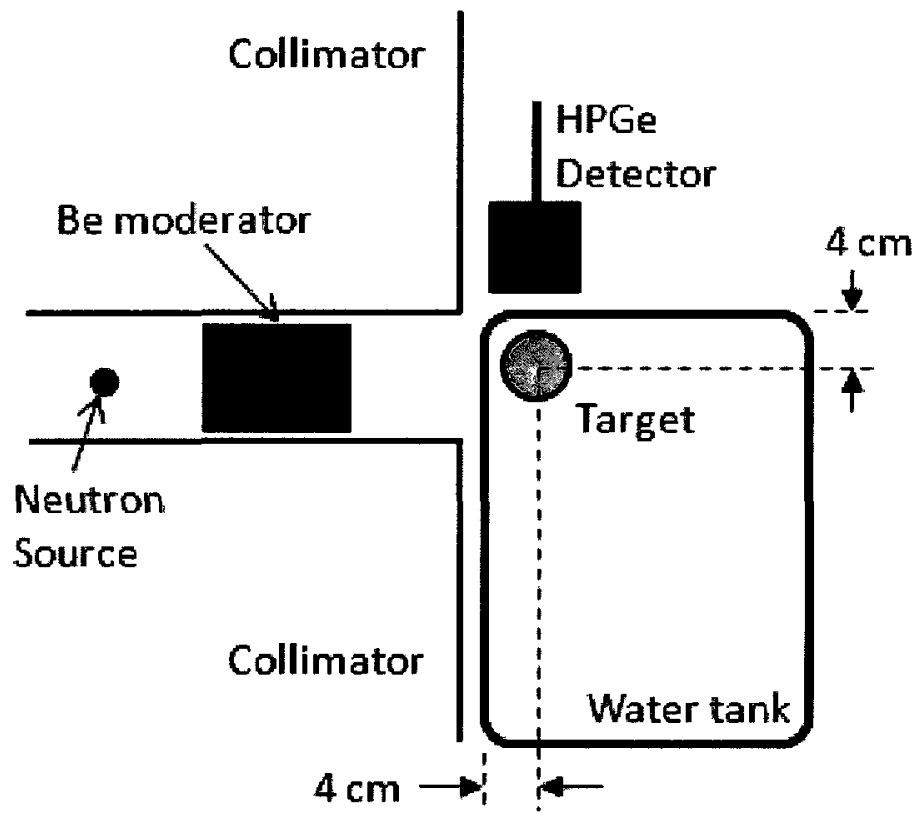

Figure 13 - Diagram of the McMaster Samarium PGNA experiment using the PuBe radioisotope neutron source

\subsubsection{Results}

The total activity calculated with MCNP for the $125 \mathrm{~g}$ target for a range of samarium concentrations is shown in Figure 14. If there was no significant neutron attenuation (i.e., flux depression), the capture rate versus samarium concentration would be linear. However, the simulation results indicate that neutron attenuation becomes significant at samarium concentrations of 150 parts per million (ppm) by mass and higher. Therefore, in order to avoid neutron attenuation around healthy bone, which may mask the presence of bone lesions, the Sm-EDTMP concentration in healthy bone should be kept below 150 ppm. 


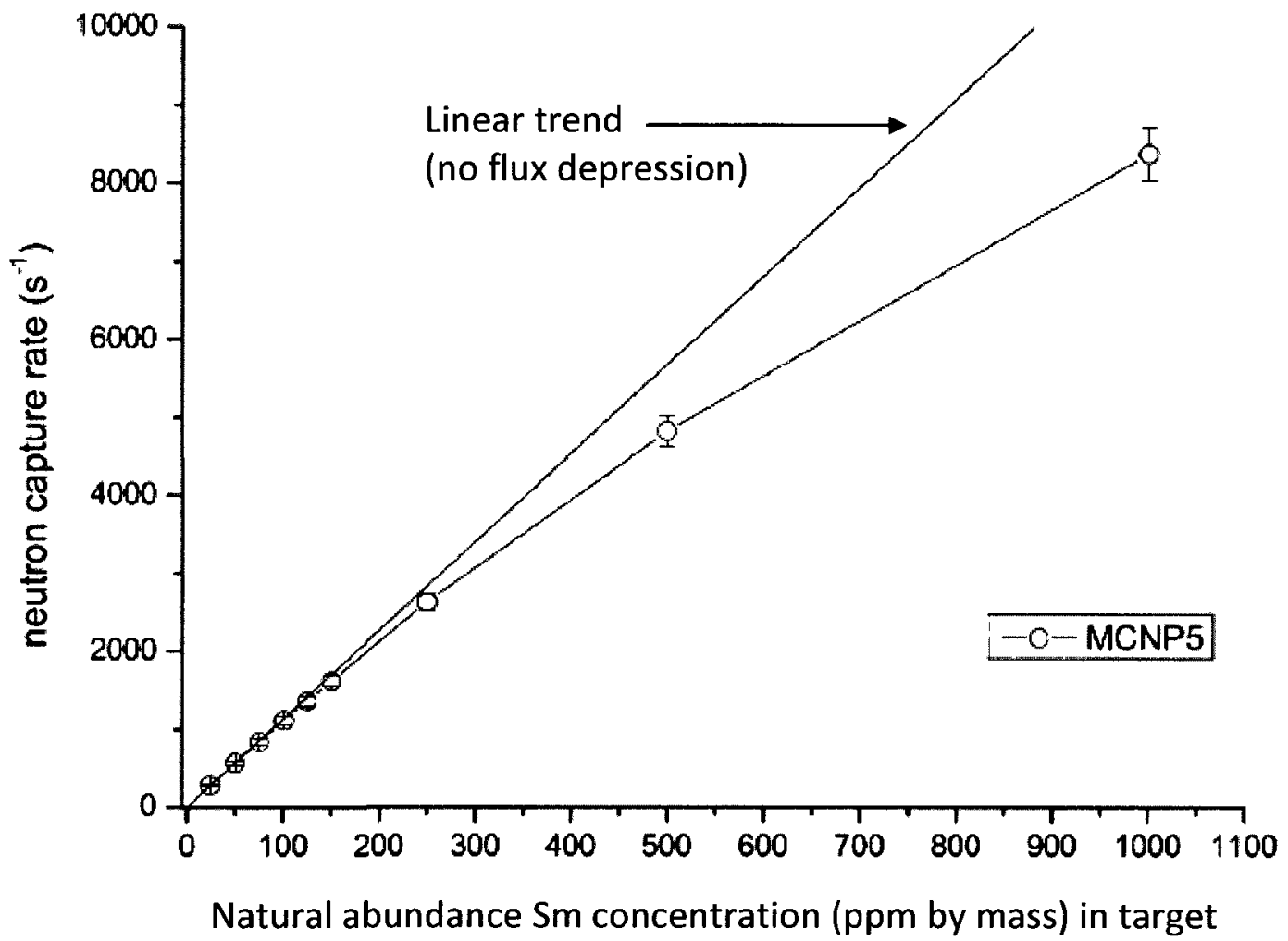

Figure 14 - Plot of neutron capture rate (or activity) versus Sm concentration in the 125 $\mathrm{mL}$ phantom, simulated with MCNP5. The linear trend line represents the change in neutron capture rate in the absence of neutron flux depression. Neutron flux depression becomes significant around $150 \mathrm{ppm}$, indicated by the divergence of the data points and the ideal linear trend.

The average thermal neutron flux in the target can be calculated by rearranging equation (5):

$$
\varphi=\frac{A}{\sigma N}
$$

For this calculation, data for the $125 \mathrm{ppm}$ Sm solution were used. The number of Sm atoms in the target, $N$, from equation (6) is: 


$$
\begin{aligned}
N & =\frac{m_{\text {target }} \times C_{S m}}{M_{S m}} \times N_{A} \\
& =\frac{(125 g \text { of solution }) \times\left(0.000125 \frac{g \text { of } S m}{g \text { of solution }}\right)}{(150.36 \mathrm{~g} \text { of } \mathrm{Sm} / \mathrm{mol} \text { of } \mathrm{Sm})} \times\left(6.02 \times 10^{23} \text { atoms } / \mathrm{mol}\right) \\
& =6.26 \times 10^{19} \text { atoms }
\end{aligned}
$$

Substituting the value of $N$ into equation (6) and using the natural abundance Sm cross-section, $\sigma$, data from Molnar (13) and activity, $A$, from Figure 14, the thermal neutron flux in the target is:

$$
\begin{aligned}
\varphi & =\frac{\left(1400 \mathrm{~s}^{-1}\right)}{\left(5.621 \times 10^{-21} \mathrm{~cm}^{2}\right) \times\left(6.26 \times 10^{19} \text { atoms }\right)} \\
& =4.0 \times 10^{3} \frac{\text { neutrons }}{\mathrm{cm}^{2} \cdot \mathrm{s}}
\end{aligned}
$$

The equivalent dose rate per unit neutron flux to the target is approximately $5.8 \times 10^{-4} \mathrm{mSv} \cdot \mathrm{h}^{-1} / \mathrm{n} \cdot \mathrm{cm}^{-2} \cdot \mathrm{s}^{-1}$ with the configuration shown in Figure 13 (43). Previous experiments at McMaster University have shown that the MNR neutron source delivers an equivalent dose rate per unit neutron flux of approximately $8.8 \times 10^{-6} \mathrm{mSv} \cdot \mathrm{h}^{-1} / \mathrm{n} \cdot \mathrm{cm}^{-2} \cdot \mathrm{s}^{-1}$ to the target (43). The reason why the PuBe source delivers almost 100 times higher dose rate per unit of neutron flux than the MNR is due to the neutron energy spectrum. The MNR neutron spectrum is approximately $99 \%$ thermalized, whereas the radioisotope source is approximately $70 \%$ thermalized (43). The $30 \%$ of the flux from the radioisotope source composed of high-energy neutrons contributes significantly to the equivalent radiation dose rate (43). To keep the equivalent dose rate low while maximizing the 
thermal neutron flux in the patient, the thermalization fraction of the neutron beam should be as high as practical.

\subsection{Objective of the 131I Experiment at The Ottawa Hospital}

The objective of the ${ }^{131}$ I Experiment was to simulate ${ }^{149} \mathrm{Sm}$ neutron-activated imaging by using radioactive ${ }^{131} \mathrm{I}$ as a proxy, since it was not feasible to construct an nSPECT prototype for this work. The primary gamma ray emission of ${ }^{131} \mathrm{I}(364 \mathrm{keV})$ is similar to that of neutron activated ${ }^{149} \mathrm{Sm}(334 \mathrm{keV})$. When a phantom containing ${ }^{131} \mathrm{I}$ is imaged by a SPECT gamma camera with the appropriate collimator, the image will represent all the energy-dependent characteristics (collimator blurring, resolution, efficiency, etc.) of an nSPECT image with ${ }^{149} \mathrm{Sm}$.

However, the ${ }^{131}$ I phantom images will not contain any of the features that come from the activation of elements other than the ${ }^{149} \mathrm{Sm}$ tracer in the patient. The neutron beam would activate hydrogen in the body by the $H(n$,gamma)D reaction. When the resulting 2.2 $\mathrm{MeV}$ gamma rays Compton-scatter in the detector, it would create background noise in the acceptance energy window. The background noise will degrade the quality of the image by making it more difficult for observers to identify lesions with a high level of confidence. Therefore, neutron-activated ${ }^{149} \mathrm{Sm}$ phantom images cannot be better than the ${ }^{131} \mathrm{I}$ phantom images; if the ${ }^{131} \mathrm{I}$ phantom images are not acceptable, then nSPECT with ${ }^{149} \mathrm{Sm}$ tracers will not be feasible. The detrimental effect of hydrogen neutron activation on image quality is assessed later in Section 4.1. 
All calculations will consider only natural abundance Sm tracers. The effects of using highly enriched ${ }^{149} \mathrm{Sm}$ tracers will be considered in the sections regarding experiment analysis.

\subsection{Experiment Description}

A phantom was constructed to represent a range of typical lesion sizes and lesionto-healthy tissue tracer uptake ratios. The phantom was filled with a liquid solution containing the ${ }^{131} \mathrm{I}$ radioisotope, which possesses a primary photon emission (364 keV) similar to that of neutron activated ${ }^{149} \mathrm{Sm}(334 \mathrm{keV})$. In Section 4.1.4, it will be shown how the ${ }^{131}$ I activity can be related to an equivalent patient dose from neutrons.

A planar imaging mode was used. Images of the phantom were acquired at a regular time interval. Any number of images from the acquired set can be added together to create one image with an equivalent total imaging time, in accordance with Poisson counting statistics (for example, three 2-minute images from the set can be summed to create a single 6-minute image).

Image quality was assessed by calculating the $\mathrm{SNR}_{\mathrm{Rose}}$ for each of the simulated lesions and comparing it with a subjective threshold. The ${ }^{131} \mathrm{I}$ phantom images were used as a reference standard of what could be achieved if hydrogen Compton noise in nSPECT was perfectly suppressed. The experimental images were also degraded by adding appropriate levels of simulated hydrogen Compton counts that we would expect to see if we were using a neutron source. The degraded images were also evaluated in the same 
way as the reference standard images. Comparing the two cases illustrates the best image quality that can be obtained for a given radiation dose, as well as the detrimental effect that hydrogen noise may have on image quality.

\subsection{Experimental Apparatus}

The experiment was conducted at The Ottawa Hospital, Civic campus. The following equipment was used for the experiment:

$>$ Siemens Symbia-T SPECT/CT imaging system in planar imaging mode (includes gamma camera heads, collimator, electronics)

Bone tracer distribution phantom. Designed to test the detection limit for lesions of varying sizes and tracer uptake ratios using planar imaging.

$>{ }^{131} \mathrm{I}$ in a water solution

Watertight container for draining the phantom

Plumbing for filling/draining the phantom (beaker, hoses, syringes, valves, etc.)

Radiation shielding and handling equipment

- Personal dosimeters

- Specialized fume hood for handling radioactive substances

- Hot storage for radioactive materials after the experiment 


\subsubsection{Gamma Camera}

The gamma camera that was used for the ${ }^{131}$ I Experiment was a Siemens SymbiaT (see Figure 15) with high energy (HE) parallel-hole collimators. The HE collimators are designed specifically for use with ${ }^{131} \mathrm{I}$. The NaI(Tl) detector crystals were $9.5 \mathrm{~mm}$ thick, which is standard for a gamma camera optimized for the $100-250 \mathrm{keV}$ energy range, with intrinsic peak efficiencies of $20 \%$ at $364 \mathrm{keV}$ and $80 \%$ at $140 \mathrm{keV}(25 ; 28)$. The system spatial resolution with the $\mathrm{HE}$ collimators is $13.4 \mathrm{~mm}$ at $10 \mathrm{~cm}$ from the detector face (25). The field of view (FOV) of the gamma camera is $533 \mathrm{~mm} \times 387 \mathrm{~mm}$. According to the calculations from Section 2.3.4.4, a gamma camera with this spatial resolution and FOV should use a $128 \times 128$ image matrix in order to optimize image quality.

The system operated in a planar imaging mode with the camera heads and patient gantry stationary. The system was configured to image with a $256 \times 256$ matrix using a $15 \%$ acceptance energy window centered at $364 \mathrm{keV}$ (i.e., an acceptance energy window between $337 \mathrm{keV}$ and $391 \mathrm{keV}$ ). The $256 \times 256$ matrix produces a sub-optimal pixel width (see Section 2.3.4.4), however, the $256 \times 256$ matrix can be reduced to the optimal $128 \times 128$ matrix, as well as a $64 \times 64$ matrix if necessary. Ninety sequential images were recorded, each with a 2 -minute acquisition time (180 minutes total imaging time). The source-to-detector separation was approximately $11 \mathrm{~cm}$ for the top detector. 


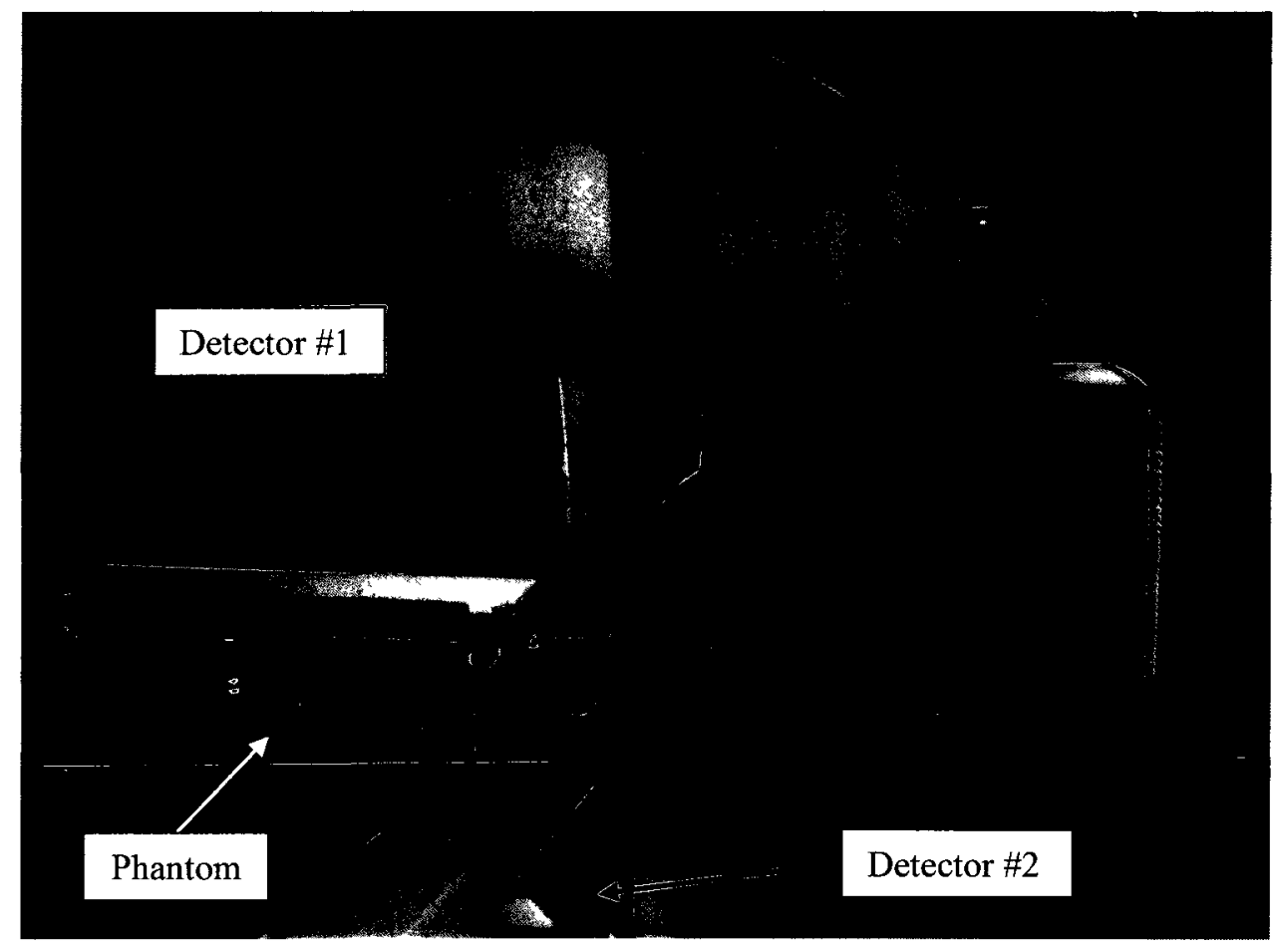

Figure 15 - The Siemens Symbia-T at The Ottawa Hospital. The phantom containing the ${ }^{131}$ I solution can be seen beneath detector 1 , on the patient gantry.

\subsubsection{Phantom}

The phantom (Figure 16, see Appendix B for more detailed drawings) was machined from Lucite (polymethyl methacrylate), which has radiological properties that are similar to muscle and fat (26). The base of the phantom had a shallow rectangular cavity containing 4 sets of cylindrical holes, with each set having a different depth. When the ${ }^{131}$ I solution was poured into this cavity, the shallow area represented the activity of healthy bone tracer uptake (background) and the holes represented lesion tracer uptake. The holes are deeper than the background region because lesions have a higher affinity for the tracer; deeper holes represent higher lesion-to-healthy uptake ratios. 


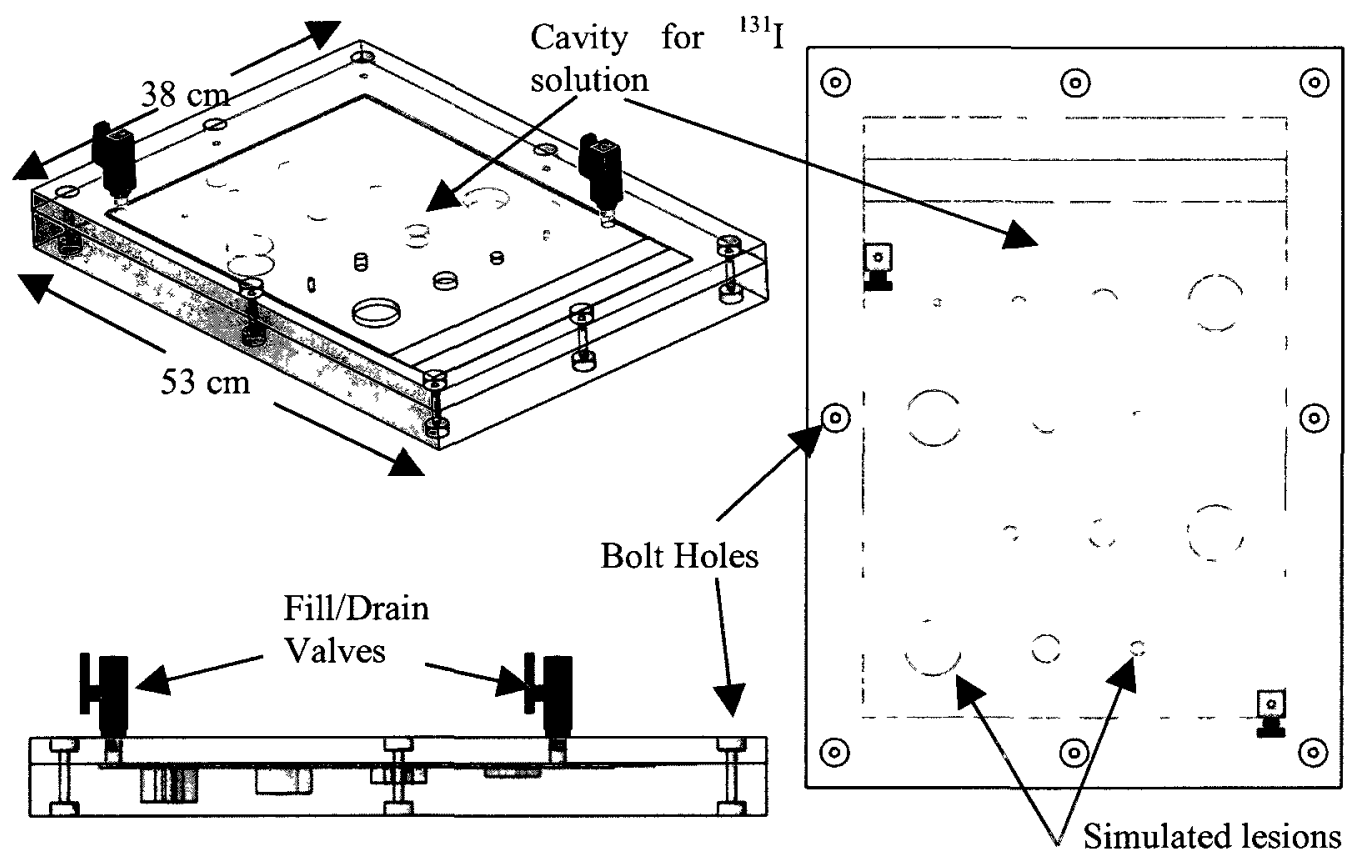

Figure 16 - Isometric (upper left), front (lower left) and top (right) views of the phantom. See Appendix B for more details on the phantom design.

The healthy-to-lesion uptake ratios examined were 1:5, 1:8, 1:12, and 1:15. The average uptake ratio is $1: 15$ for ${ }^{153} \mathrm{Sm}$-EDTMP, however, data for the other ratios may be helpful in the future for applications that use other carrier molecules in place of EDTMP (23; 21). From the gamma camera perspective, these ratios will appear to be $1: 3,1: 4.5$, 1:6.5, and 1:8 for reasons illustrated in Figure 17 (21). For the phantom in Figure 16, a $1.5 \mathrm{~mm}$ depth of ${ }^{131} \mathrm{I}$ solution represents the activity per unit area on the surface of healthy bone. Therefore, the shallow square section will contain $3 \mathrm{~mm}$ of ${ }^{131} \mathrm{I}$ solution, and the hole depths would be $21,16.5,10.5$, and $6 \mathrm{~mm}$. However, because the simulated lesions in the phantom have considerable depth, the gamma ray attenuation in the solution becomes significant, so the depth of the tumors must be increased slightly in 
order to achieve the activity ratios mentioned above (the corrected depths are 25,19 , 11.5, and $6.5 \mathrm{~mm}$ ). Appendix $\mathrm{C}$ contains a sample calculation for determining the attenuation-corrected hole depth. The simulated lesion diameters are $4,2,1$, and $1 / 2 \mathrm{~cm}$.
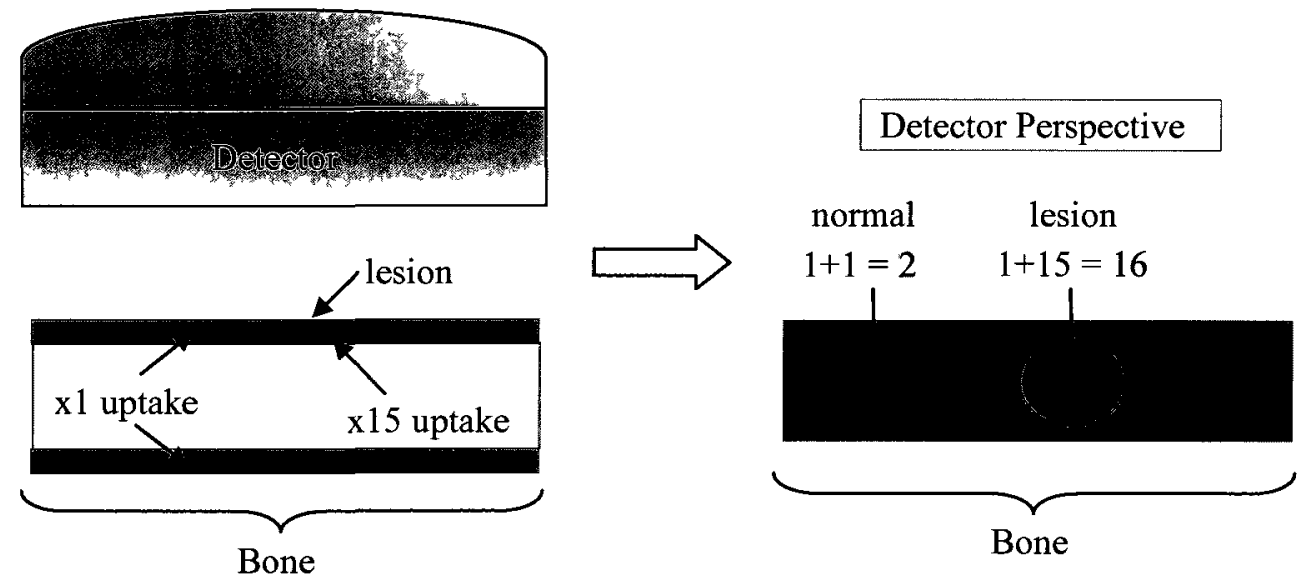

Figure 17 - The ${ }^{149}$ Sm-EDTMP tracer will accumulate in the surface of bone. The image on the left is an illustration of a cross-section of bone that contains a lesion and has accumulated some of the tracer. From the perspective of a detector above the bone in planar imaging mode, the activity will appear as it does in the illustration on the right. The tumor, at $15 \mathrm{x}$ normal uptake, only appears to have $8: 1$ contrast.

The top section of the phantom was a $1.9 \mathrm{~cm}$ thick acrylic sheet that sealed the phantom and provided a scattering medium for the photons emitted by the ${ }^{131}$ I solution. The top was held in place by eight bolts and was water-tight. The two valves provided a way to fill and drain the phantom. The volume of the cavity in the phantom was $470 \mathrm{~mL}$. After the experiment, the ${ }^{131} \mathrm{I}$ solution was drained from the phantom and the solution was placed in hot storage until the radioactivity decayed to safe levels. 


\subsubsection{I Solution}

Iodine-131 is a radioactive isotope with a physical half-life of 8.04 days (51). It emits both beta and gamma radiation; the energies of the radiation are given in Table 3 . Iodine-131 is useful for a variety of medical procedures and is acquired in a concentrated form, typically in the range of $\sim 37 \mathrm{MBq} / \mathrm{mL}(1 \mathrm{mCi} / \mathrm{mL})$.

Table 3 - Some radiation energies and emission probabilities (\%) for ${ }^{131} \mathrm{I}(51)$

\begin{tabular}{|c|c|}
\hline Gammas & Maximum beta energies \\
\hline $0.723 \mathrm{MeV}(1.8 \%)$ & $0.248 \mathrm{MeV}(2.1 \%)$ \\
$0.637 \mathrm{MeV}(7.3 \%)$ & $0.334 \mathrm{MeV}(7.4 \%)$ \\
$0.364 \mathrm{MeV}(81.2 \%)$ & $0.606 \mathrm{MeV}(89.3 \%)$ \\
$0.284 \mathrm{MeV}(6.1 \%)$ & \\
$0.080 \mathrm{MeV}(2.6 \%)$ & \\
\hline
\end{tabular}

The total activity of ${ }^{131} \mathrm{I}$ required for the ${ }^{131} \mathrm{I}$ solution in the phantom was calculated based on the expected activity from natural abundance Sm-EDTMP nSPECT. The ${ }^{131} \mathrm{I}$ phantom is designed to simulate the activity per unit area of bone in planar imaging, therefore equation 5 must be modified in order to calculate the expected activity per unit area from natural abundance Sm-EDTMP PGNA:

$$
\frac{A_{334 \mathrm{keV}}}{a}=\sigma_{334 \mathrm{keV}} \varphi \frac{N}{a}
$$

where $A_{334 \mathrm{keV}}$ is the activity of the $334 \mathrm{keV}$ emission from Sm PGNA, $a$ is a unit area (1 $\mathrm{cm}^{2}$ ), $\sigma_{334 \mathrm{keV}}$ is the atomic Maxwellian average (at $298 \mathrm{~K}$ ) cross-section for an (n,gamma) reaction producing a $334 \mathrm{keV}$ gamma emission, $\varphi$ is the Maxwellian (at $298 \mathrm{~K}$ ) neutron 
flux, and $N / a$ is the expected number of ${ }^{149} \mathrm{Sm}$ atoms per unit area of healthy bone, and is defined as:

$$
\begin{aligned}
\frac{N}{a} & =\frac{c_{S m}}{M_{S m}} \times N_{A} \times \rho_{\text {bone }} \times d_{\text {bone }} \times \%{ }^{149} \text { Sm natural abundance } \\
& =\frac{\left(0.000125 \frac{\mathrm{gof} \mathrm{Sm}}{\mathrm{gof} \text { tissue }}\right)}{150.36 \mathrm{~g} / \mathrm{mol}}\left(6.022 \times 10^{23} \text { atoms } / \mathrm{mol}\right)\left(1.65 \frac{\mathrm{g}}{\mathrm{cm}^{3}}\right)(1.5 \mathrm{~cm})(0.138) \\
& =1.7 \times 10^{17} \frac{149 \mathrm{Sm} \text { atoms }}{\mathrm{cm}^{2}}
\end{aligned}
$$

where $C_{S m}$ is the fraction by weight of $\mathrm{Sm}$ in bone, $M_{S m}$ is the average molar mass of Sm, $N_{A}$ is Avogadro's number, $\rho_{\text {bone }}$ is the average density of bone, and $d_{\text {bone }}$ is the thickness of bone. An average bone thickness, $d_{\text {bone }}$, of $1.5 \mathrm{~cm}$ was assumed, which is slightly thinner than the average thickness of the narrowest part of a human Femur (52). A natural abundance $\mathrm{Sm}$ concentration, $C_{S m}$, of $125 \mathrm{ppm}$ (by mass) in bone was chosen for two reasons. First, the absolute mass of Sm in the body $(1.6 \mathrm{~g})$ was well below the $4.7 \mathrm{~g}$ limit for oral administration from Section 2.5.2, assuming a $70 \mathrm{~kg}$ person, of which an average of $18 \%$ is bone mass (53). Second, there would be negligible neutron flux depression at a concentration of $125 \mathrm{ppm}$. For an average bone density of $1.65 \mathrm{~g} / \mathrm{cm}^{3}(26)$, the mass per unit area for the bone model is $\sim 2.5 \mathrm{~g} / \mathrm{cm}^{2}$ compared with $\sim 4 \mathrm{~g} / \mathrm{cm}^{2}$ for the McMaster phantom. The mass per unit area for a target describes the quantity of material that a beam must pass through. A higher mass per unit area will attenuate the beam more than a lower mass per unit area. Because the McMaster phantom did not experience significant neutron flux depression (i.e., neutron attenuation) at $125 \mathrm{ppm} \mathrm{Sm}$ and $4 \mathrm{~g} / \mathrm{cm}^{2}$, we can 
assume that there would be negligible neutron flux depression with this bone model at 2.5 $\mathrm{g} / \mathrm{cm}^{2}$

The value of $\varphi$ in equation 8 was assumed to be two orders of magnitude higher than the thermal neutron flux in the target from the McMaster Experiment (see Section 3.1.2), which is within the capabilities of the electric neutron source envisioned for nSPECT (12). $A_{334 \mathrm{kev}} / a$ from equation 8 is:

$$
\begin{aligned}
\frac{A_{334 \mathrm{keV}}}{a} & =\left(4.92 \times 10^{-20} \mathrm{~cm}^{2}\right)\left(4.0 \times 10^{5} \frac{\text { neutrons }}{\mathrm{cm}^{2} \cdot \mathrm{s}}\right)\left(1.7 \times 10^{17} \frac{{ }^{149} \mathrm{Sm} \text { atoms }}{\mathrm{cm}^{2}}\right) \\
& =3300 \frac{B q}{\mathrm{~cm}^{2}}
\end{aligned}
$$

The total ${ }^{131}$ I solution activity was calculated by rearranging the equation for the ${ }^{131}$ I activity per unit area in the simulated healthy bone region of the phantom:

$$
\begin{aligned}
\frac{A_{364 \mathrm{keV}}}{a} & =\frac{A_{I-131} \times P(364 \mathrm{keV})}{V_{\text {Phantom }}} \times d_{\text {Healthy bone }} \\
A_{I-131} & =\frac{\frac{A_{364 \mathrm{keV}}}{a}}{d_{\text {Healthybone }}} \times V_{\text {Phantom }} \times \frac{1}{P(364 \mathrm{keV})}
\end{aligned}
$$

where $A_{364 \mathrm{keV}} / a$ is equal to $A_{334 \mathrm{keV}} / a$ from equation $8, d_{\text {Healthy bone }}$ is the depth of ${ }^{131} \mathrm{I}$ solution in the simulated healthy bone section of the phantom, $V_{\text {Phantom }}$ is the total volume of the phantom cavity, and $P(364 \mathrm{keV})$ is the probability of a $364 \mathrm{keV}$ gamma emission from an ${ }^{131} \mathrm{I}$ decay. The total ${ }^{131}$ I solution activity from equation 10 is: 


$$
\begin{aligned}
A_{I-131} & =\frac{3300 \frac{\mathrm{Bq}}{\mathrm{cm}^{2}}}{0.30 \mathrm{~cm}} \times 470 \mathrm{~cm}^{3} \times \frac{1}{0.812} \\
& =6.4 \times 10^{6} \mathrm{~Bq}
\end{aligned}
$$

The total ${ }^{131}$ I activity required to simulate natural abundance Sm-EDTMP neutron activation was $6.4 \mathrm{MBq}(0.172 \mathrm{mCi})$. The $6.4 \mathrm{MBq}$ of ${ }^{131} \mathrm{I}$ was diluted with $470 \mathrm{~mL}$ of tap water and was dyed red, which made it easier to verify that all of the phantom features were properly filled and free of trapped air bubbles. The red dye would also facilitate cleanup in the event of a spill.

\subsection{Results}

The output of the SPECT imaging system is a set of 90 matrices (i.e., images), each with a $256 \times 256$ pixel array. Each pixel is represented by a matrix element that contains the number of counts that were detected in the acceptance energy window at that location in the crystal over the course of the two-minute integration time. In Figure 18, a single two-minute image is shown alongside the 180-minute image, which is a composite of all 90 images. 


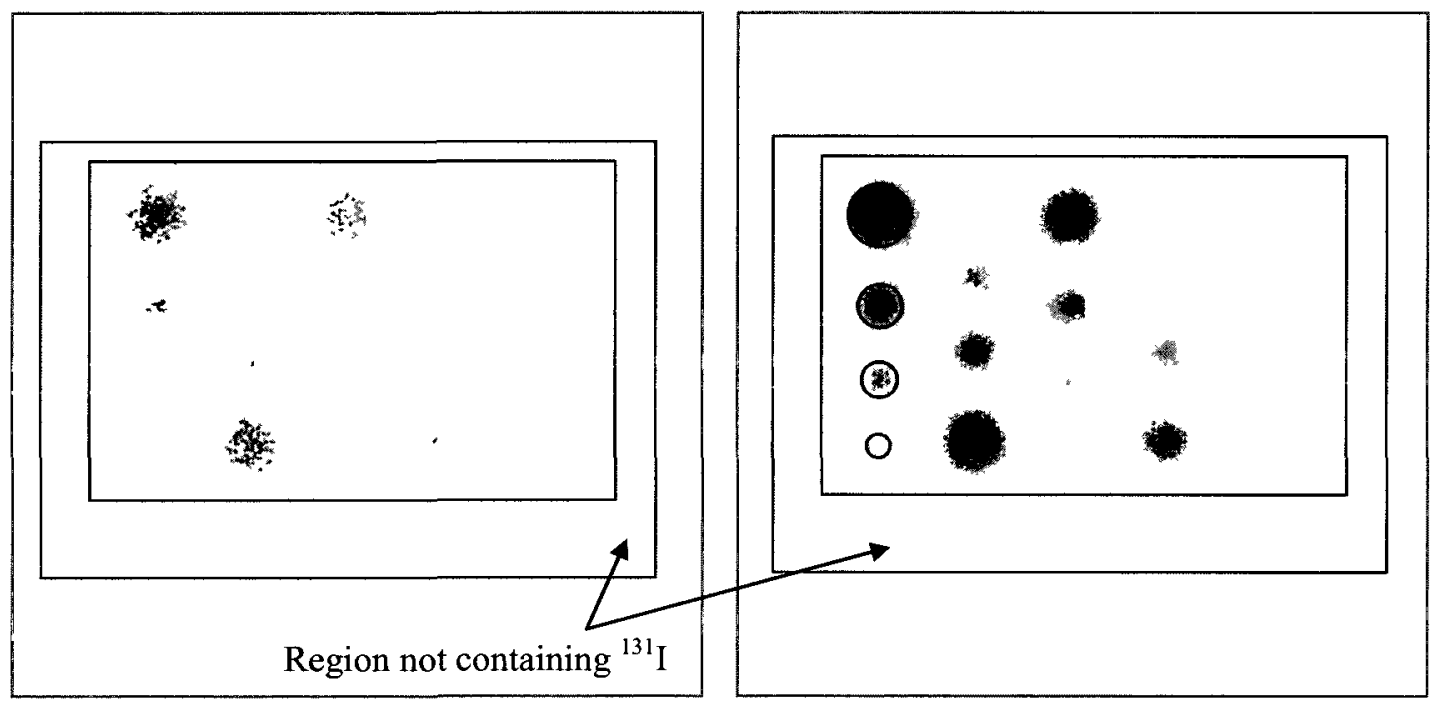

Figure 18 - Images of the ${ }^{131}$ I phantom produced by the Symbia T SPECT system. The image on the left is a 2 -minute integration ( $31 \mathrm{k}$ total counts). The image on the right is a 180-minute integration, which is the sum of all 90 two-minute images ( $\sim 2.8$ million counts). The image matrix size is $256 \times 256$.

The ideal image matrix is $128 \times 128$ when imaging ${ }^{131} \mathrm{I}$ with this gamma camera configuration (see Sections 2.3.4.4 and 3.4.1). It is possible to reduce the size of a matrix from $256 \times 256$ to $128 \times 128$ to $64 \times 64$ by summing pairs of adjacent columns and rows. A 2-minute image of the $128 \times 128$ and $64 \times 64$ matrix sizes is shown in Figure 19. 

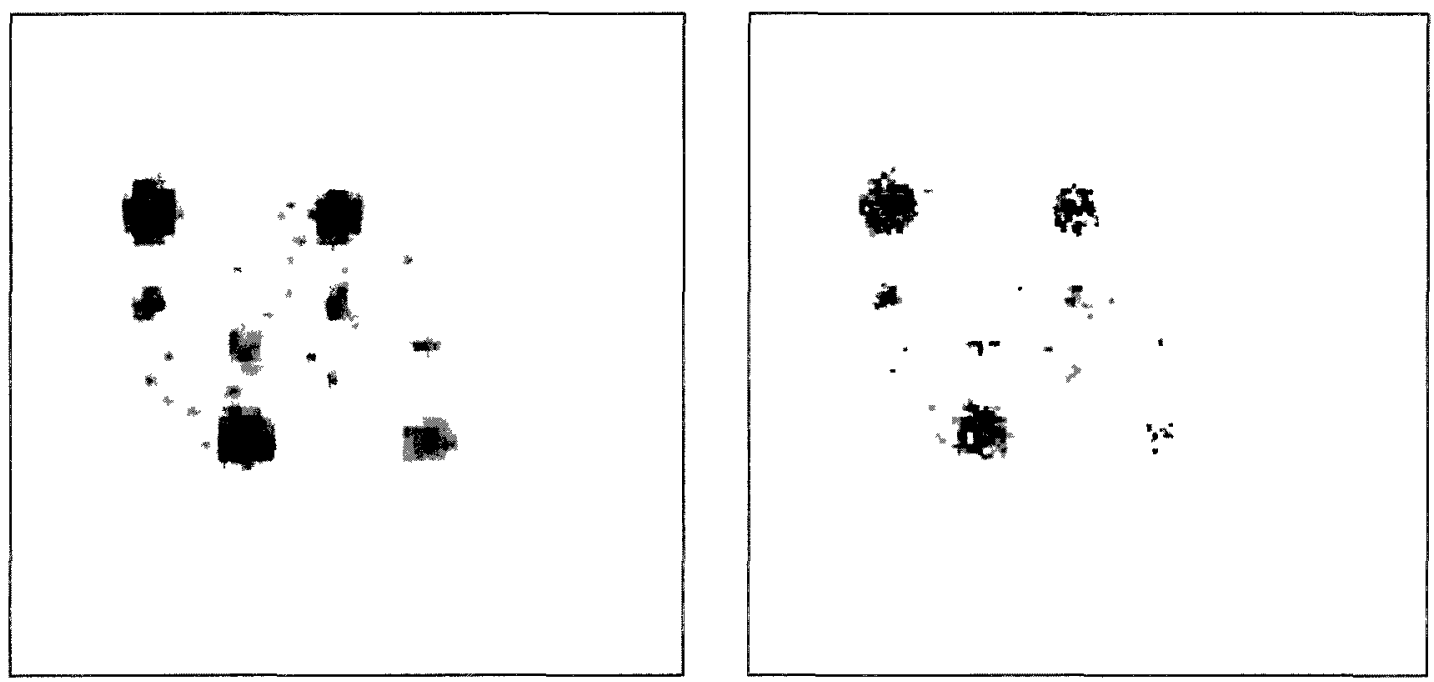

Figure 19 - Images of the ${ }^{131} \mathrm{I}$ phantom for a 2 -minute acquisition time with reduced matrix sizes. The image on the left is $64 \times 64$ pixels and the image on the right is $128 \times 128$ pixels.

Observing the images in Figure 18, one can see some of the energy- and collimator-dependent effects of imaging. Low levels of activity are seen in regions around the edge of the phantom, which do not contain any ${ }^{131} \mathrm{I}$; this is particularly pronounced in the 2-minute image of Figure 18. Those counts are a result of collimator penetration by photons originating from the central regions of the phantom. The edges of the lesions are blurred due to the finite field-of-view and edge-penetration of the parallel collimator holes. The $1 \mathrm{~cm}$ and $0.5 \mathrm{~cm}$ simulated lesions have lower lesion-tobackground contrast than the $4 \mathrm{~cm}$ and $2 \mathrm{~cm}$ simulated lesions because they are significantly smaller than the $13.4 \mathrm{~mm}$ spatial resolution of the system, as discussed in Section 2.3.4.3. 


\section{CHAPTER 4: DATA ANALYSIS}

The results of the ${ }^{131}$ I Experiment are an estimate of an ideal case of nSPECT planar bone imaging with ${ }^{149}$ Sm-EDTMP. In practice, nSPECT images will be confounded by the Compton background from concomitant neutron activation of hydrogen in the body through the $H(n$, gamma)D reaction. By understanding the significance and nature of the impact of hydrogen noise on ${ }^{149} \mathrm{Sm}$ nSPECT images, it may be possible to develop techniques to minimize its negative effects. The following subsections describe the methods used to:

- estimate and model the hydrogen Compton background in the acceptance energy window

- estimate the patient radiation dose from neutrons

- add the hydrogen background to the ${ }^{131}$ I Experiment images

- $\quad$ and evaluate the noisy images

\subsection{Hydrogen Noise and Patient Equivalent Dose}

Hydrogen noise refers to the PGNA of hydrogen atoms in and around target tissue, which produces high energy gamma rays that Compton scatter in the detector and produce counts that are uncorrelated to the Sm-EDTMP activity distribution. In order to estimate the quantity of hydrogen noise that will be present, assumptions must be made regarding the quantity and geometry of hydrogen-rich tissue surrounding the target and 
the resulting neutron flux distribution through that tissue. Knowing the neutron flux in the target tissue (discussed in Section 3.4.3), it is possible to estimate the neutron flux in the surrounding tissue and, therefore, the equivalent dose rate to that tissue and the concomitant hydrogen activity. The hydrogen activity data must be combined with the energy-dependent gamma camera efficiency and peak-to-Compton background ratio to determine the number of counts due to hydrogen in the $334 \mathrm{keV}$ acceptance energy window. Each of these steps are explored in detail in the following subsections.

\subsubsection{Tissue Model}

The tissue geometry chosen for this analysis was that of a human thigh, which would be a worst-case scenario for bone imaging in terms of radiation dose to the patient. This will become apparent further in the analysis. The tissue model was only a rough approximation to a thigh because the ${ }^{131} \mathrm{I}$ phantom was not anthropomorphic. The only semblance between the tissue model and an actual human thigh were as follows:

- The bone containing the ${ }^{149} \mathrm{Sm}$ tracer (i.e., ${ }^{131} \mathrm{I}$ solution in the phantom) was located in the center of the surrounding tissue

- The surrounding tissue had thickness of $12 \mathrm{~cm}$, consistent with average measurements of human thighs (52)

Figure 20 is an illustration of the tissue model. The neutron flux was assumed to be homogenous in the planes parallel to the detector. For the purpose of this analysis, the tissue model was divided into 12 layers (each $1 \mathrm{~cm}$ thick) and for each layer the average 
neutron flux and hydrogen activity was calculated (see Sections 4.1.2 and 4.1.3). The tissue was modeled as pure $\mathrm{H}_{2} \mathrm{O}$, which is commonly used to approximate human tissue due to their similar radiological properties (26). The mass of water present was assumed to be $17.5 \mathrm{~kg}$, which is $1 / 4$ of the mass of an average $70 \mathrm{~kg}$ person; and this is the average mass of material that would be within the field of view of a typical $40 \mathrm{~cm} \times 55 \mathrm{~cm}$ Gamma Camera (19). Therefore, each $1 \mathrm{~cm}$ layer contains approximately $9.8 \times 10^{25}$ hydrogen atoms and, with a Maxwellian average (at $298 \mathrm{~K}$ ) neutron absorption crosssection of $3.65 \times 10^{-25} \mathrm{~cm}^{2}$ per atom (see Table 2 in Section 2.4.3), has a macroscopic PGNA cross-section of $36 \mathrm{~cm}^{2}$.

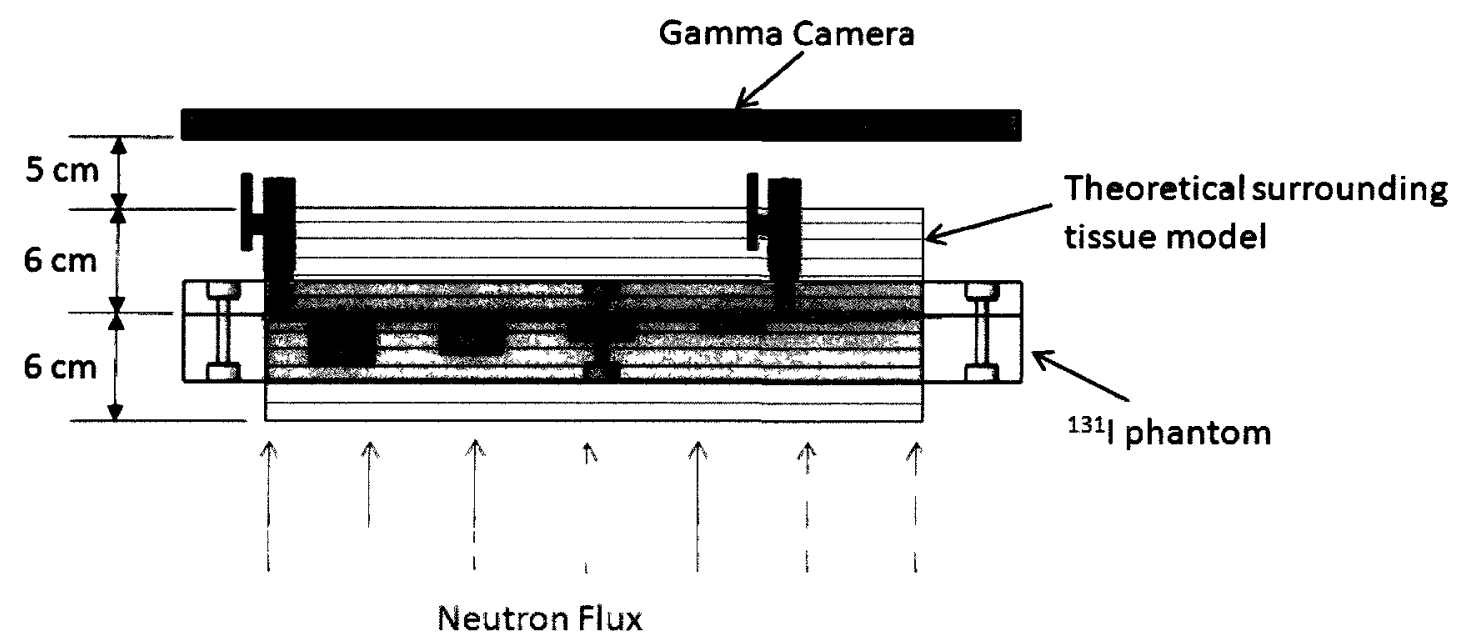

Figure $20-$ An illustration of a water volume that is meant to mimic the muscle and fatty tissue in a human thigh around the ${ }^{131}$ I phantom, which mimics lesions on bone. On one side, the neutron flux enters the tissue and on the other, the Gamma Camera is positioned to detect the resulting PGNA gamma rays. The active layer in the ${ }^{131} \mathrm{I}$ phantom is $11 \mathrm{~cm}$ from the detector face and the tissue model is $5 \mathrm{~cm}$ from the detector face. 


\subsubsection{Neutron Flux Distribution in the Tissue Model}

The average neutron flux in each layer was calculated based on MCNP5 Monte Carlo simulation data provided by Heliocentric Technologies. The simulation used a fast neutron source surrounded by $1 \mathrm{~cm}$ thick concentric spheres of high-density polyethylene (chains of $\mathrm{C}_{2} \mathrm{H}_{4}$ ) with approximately the same density $\left(\rho_{\mathrm{HDPE}}=0.984 \mathrm{~g} / \mathrm{cm}^{3}\right.$ ) as water. A plot of the simulation data is shown in Figure 21. In order to achieve a neutron flux of 4.0 $\mathrm{x} 10^{5} \mathrm{n} \mathrm{cm}^{-2} \mathrm{~s}^{-1}$ in the target (see Section 3.4.3) at a depth of $6 \mathrm{~cm}$ in the tissue model, the patient will have to be exposed to over double that target flux at the skin surface.

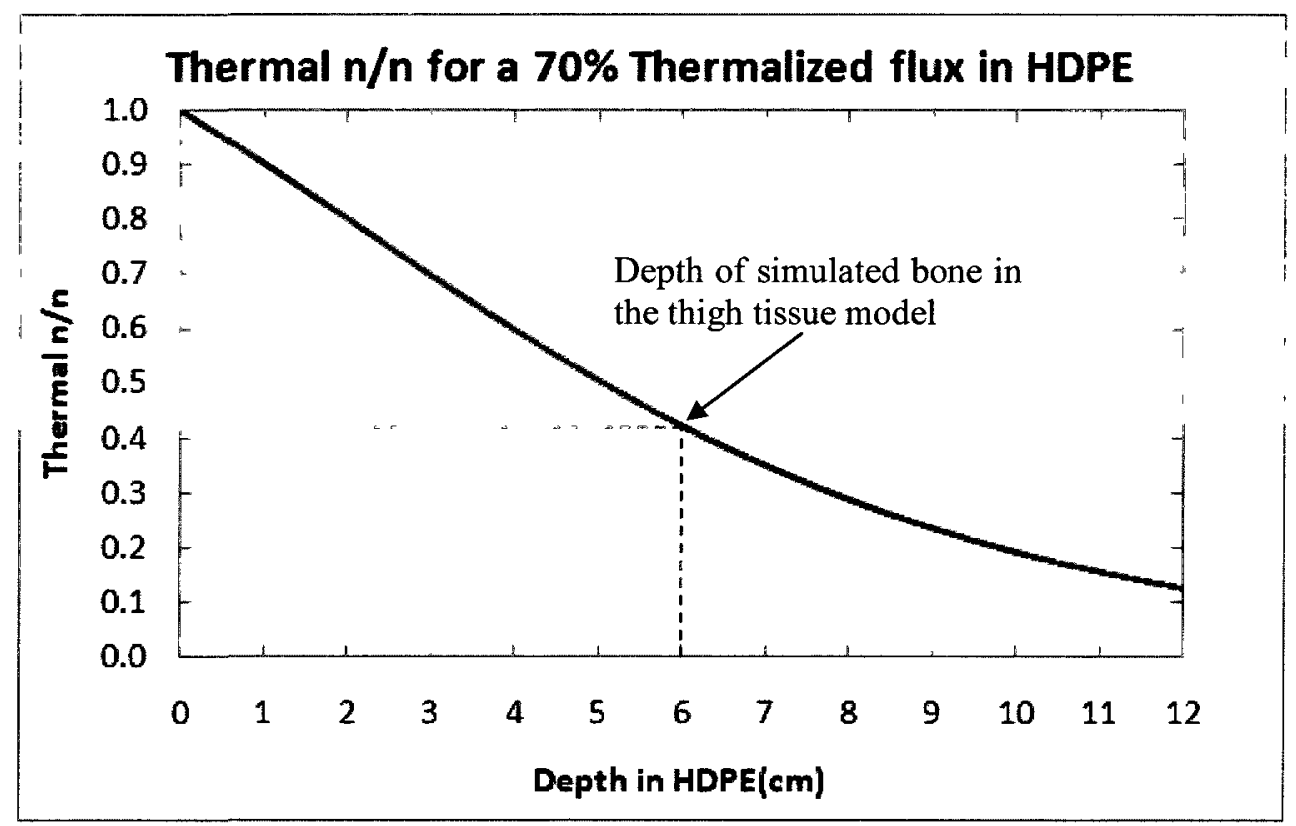

Figure 21 - MCNP5 simulation data for a fast neutron source surrounded by concentric spheres of HDPE at $0.984 \mathrm{~g} / \mathrm{cm}^{3}$. The flux corresponding to depth $0 \mathrm{~cm}$ is $70 \%$ thermalized, matching the flux characteristics of the PuBe radioisotope neutron source used in the McMaster experiment (Section 3.1). Neutron flux is normalized relative to the flux at $0 \mathrm{~cm}$, which is the skin surface in the thigh tissue model. 
The data in Figure 21 help to illustrate why the thigh imaging model represents the worst case dose scenario. In any other bone imaging scenario (skull, ribs, arms, etc.), the bone will be close to the surface rather than deeply buried in the center of the surrounding tissue. Since the half value layer of $334 \mathrm{keV}$ photons in water $(6 \mathrm{~cm})$ is larger than the half value layer of thermal neutrons in water $(5 \mathrm{~cm}$ for $70 \%$ thermalization, lower for fluxes with higher thermalization), thermal neutrons experience more attenuation than photons in tissue. For this reason, it is better to expose bones to neutrons through less tissue and image photons through more tissue, if possible, which will result in a lower equivalent radiation dose. Thus, the thigh model represents the worst case dose scenario.

\subsubsection{Compton Background from 2.2 MeV Photons}

Gamma Cameras are not designed for use with photons as energetic as $2.2 \mathrm{MeV}$, so there is very limited data on detector efficiency and the detected energy spectrum resulting from $\mathrm{MeV}$-level interactions (16). Simulation data for $2.2 \mathrm{MeV}$ photon interactions with a gamma camera were obtained from literature; however, the simulation work did not include the gamma camera collimators (54). Therefore, all calculations in this section do not consider the effect of the High Energy (HE) collimators used in the

${ }^{131}$ I phantom imaging experiment. It is expected that the HE collimator would have a beneficial effect by reducing the number of $2.2 \mathrm{MeV}$ photons reaching the detector. For example, using equation 4 and lead attenuation coefficient data from Section 2.4.2, we 
can calculate that the $59.7 \mathrm{~mm}$ thick HE collimator has a $95 \%$ probability of scattering a 2.2 $\mathrm{MeV}$ gamma ray. This assumes the gamma ray path is perpendicular to the collimator face and it does not enter one of the collimator holes. There is also a significant probability that the scattering angle of the $2.2 \mathrm{MeV}$ gamma ray will be directed away from the detector crystal (26).

The simulation was part of a research project to determine the feasibility of using uncollimated commercial gamma cameras as high-sensitivity spectrometers for detecting and quantifying isotopes that would likely be involved in a radiological emergency, such as an improvised nuclear explosive. The simulation data were produced using the GEANT $-4^{6}$ Monte Carlo code developed by CERN (54). The detector was a $50 \times 40 \times 1$ $\mathrm{cm}^{3} \mathrm{NaI}(\mathrm{Tl})$ gamma camera and the gamma rays originated from a point source located 1 meter from the detector (54). The gamma energies for the point source were based on those from uranium ore, including one gamma ray at $2.204 \mathrm{MeV}$, which nearly identical to the energy of hydrogen PGNA $(13 ; 54)$.

Detector efficiency values are highly dependent on the source position and geometry, therefore it is necessary to modify the values provided by (54) before they can be used in this analysis. The Efficiency Transfer for Nuclide Activity measurement

\footnotetext{
${ }^{6}$ GEANT-4 is a Monte Carlo toolkit used to simulate the passage of particles through matter. It is used for high energy, nuclear and accelerator physics, as well as for studies in medical and space science. GEANT-4 is particularly useful for simulating radiation detectors, such as those used in CT, SPECT, and PET imaging. For more information, see the GEANT-4 homepage at: http://www.geant4.org/geant4/index.shtml
} 
$(\text { ETNA })^{7}$ software, provided by the Laboratoire National Henri Becquerel upon request, was used to calculate the detector efficiency for the tissue model from Section 4.1.2 based on the point source efficiency from (54). For each of the 12 slices in the tissue model, the full energy peak efficiency (FEPE) of the gamma camera at $2.2 \mathrm{MeV}$ was calculated (details of the ETNA setup parameters are provided in Appendix D). The FEPE is the fraction of photons, out of all photons emitted from the source, which deposit all of their energy in the detector (i.e., FEPE $\leq 1)$. The greater the separation between the detector and the source, the lower the detector FEPE will be. An absorbing medium between the source and detector will also reduce the FEPE, and the detector FEPE is inversely proportional to the gamma ray energy, because high energy photons are more likely to Compton scatter in the detector. The ETNA software accounts for all of these factors.

The FEPE at 2.2 MeV for each slice is shown in Table 4, along with the average Maxwellian neutron flux (at $298 \mathrm{~K}$ ) and the gamma camera full energy peak counts per second. This last parameter was calculated by multiplying the macroscopic hydrogen PGNA cross-section (from Section 4.1.1) with the neutron flux (from Section 4.1.2) and

\footnotetext{
${ }^{7}$ ETNA uses geometry and photon attenuation calculations to determine the detector efficiency for a variety of radiation source-detector separations and radiation source geometries, based on a reference efficiency for a well defined geometry. For example, ETNA can use detector efficiency values for a point source at $30 \mathrm{~cm}$ from the detector to calculate the detector efficiency values of a cylindrical source at $100 \mathrm{~cm}$ from the detector. ETNA possesses other useful tools as well. For more information, consult the ETNA homepage at:

http://www.nucleide.org/Gamma spectro/logiciels_de traitement.htm
} 
the FEPE at $2.2 \mathrm{MeV}$ for that slice. The total gamma camera count rate in the $2.2 \mathrm{MeV}$ peak is $5.8 \times 10^{5} \mathrm{~s}^{-1}$

Table $4-$ The average parameters for each of the twelve layers of the tissue model described in Section 4.1.1. Depth position $0 \mathrm{~cm}$ corresponds to the point closest to the neutron source (i.e., furthest from the detector). The highlighted row corresponds to the position of the ${ }^{131}$ I phantom activity.

\begin{tabular}{|cccc|}
\hline $\begin{array}{c}\text { Depth position }(\mathrm{cm}) \\
\text { in tissue model }\end{array}$ & $\begin{array}{c}\text { Detector FEPE } \\
@ 2.2 \mathrm{MeV}\end{array}$ & $\begin{array}{c}\text { Average } \phi\left(\mathrm{n} / \mathrm{cm}^{2} / \mathrm{s}\right) \\
\text { Aamma Camera count } \\
\text { rate }\left(\mathrm{s}^{-1}\right) @ 2.2 \mathrm{MeV}\end{array}$ \\
\hline $0-1$ & $1.73 \times 10^{-3}$ & $9.0 \times 10^{5}$ & $5.5 \times 10^{4}$ \\
$1-2$ & $1.94 \times 10^{-3}$ & $8.0 \times 10^{5}$ & $5.6 \times 10^{4}$ \\
$2-3$ & $2.19 \times 10^{-3}$ & $7.1 \times 10^{5}$ & $5.5 \times 10^{4}$ \\
$3-4$ & $2.47 \times 10^{-3}$ & $6.1 \times 10^{5}$ & $5.4 \times 10^{4}$ \\
$4-5$ & $2.81 \times 10^{-3}$ & $5.2 \times 10^{5}$ & $5.2 \times 10^{4}$ \\
$5-6$ & $3.20 \times 10^{-3}$ & $4.4 \times 10^{5}$ & $5.0 \times 10^{4}$ \\
6 & & $4.0 \times 10^{5}$ & \\
$6-7$ & & (from Section 3.4.3) & \\
$7-8$ & $3.67 \times 10^{-3}$ & $3.6 \times 10^{5}$ & $4.8 \times 10^{4}$ \\
$8-9$ & $4.24 \times 10^{-3}$ & $3.0 \times 10^{5}$ & $4.5 \times 10^{4}$ \\
$9-10$ & $4.93 \times 10^{-3}$ & $2.5 \times 10^{5}$ & $4.3 \times 10^{4}$ \\
$10-11$ & $5.79 \times 10^{-3}$ & $2.0 \times 10^{5}$ & $4.1 \times 10^{4}$ \\
$11-12$ & $6.89 \times 10^{-3}$ & $1.6 \times 10^{5}$ & $4.0 \times 10^{4}$ \\
\hline Total & $8.34 \times 10^{-3}$ & $1.3 \times 10^{5}$ & $3.9 \times 10^{4}$ \\
\hline
\end{tabular}

The energy resolution (FWHM) of a photon peak measured with a $\mathrm{NaI}(\mathrm{Tl})$ gamma camera can be conservatively estimated with the empirical equation (54):

$$
F W H M(\%)=7 \% \times\left(\frac{662 \mathrm{keV}}{\text { energy }}\right)^{0.34}
$$

where $7 \%$ is the FWHM for a $662 \mathrm{keV}$ photon from ${ }^{137} \mathrm{Cs}$, which was used as a reference source for developing the equation, and energy refers to the photon energy of interest (54; 
55). At $2.2 \mathrm{MeV}$, the peak FWHM calculated from equation 11 is $\sim 4.7 \%$ (or $100 \mathrm{keV}$ ), which is equivalent to a standard deviation of approximately $43 \mathrm{keV}$. With the peak center count rate per $\mathrm{keV}$ to Compton count rate per $\mathrm{keV}$ ratio of approximately 0.40 (54), the Compton count rate per $\mathrm{keV}$ was calculated with the equation:

$$
\begin{aligned}
\text { Compton } \frac{c}{s \cdot k e V}= & \left(\frac{1}{\sqrt{2 \pi \sigma^{2}}} e^{-(x-u)^{2} /\left(2 \sigma^{2}\right)}\right) \times\left(\text { total } 2.2 \mathrm{MeV} \text { peak } \frac{\mathrm{c}}{\mathrm{s}}\right) \times(0.40) \\
& =\left(9.18 \times 10^{-3} \mathrm{keV}^{-1}\right) \times\left(5.8 \times 10^{5} \mathrm{c} / \mathrm{s}\right) \times(0.40) \\
& =2.1 \times 10^{3} \mathrm{c} \mathrm{s}^{-1} \mathrm{keV}^{-1}
\end{aligned}
$$

The first factor on the right of equation 12 is the probability density function of a normal distribution (i.e., the full energy peak at $2.2 \mathrm{MeV}$ ), where $u$ is the center of the distribution $(2.2 \mathrm{MeV}), x$ is some point in the distribution (in this case, $x=u$ to find the count rate per $\mathrm{keV}$ in the peak center), and $\sigma$ is the standard deviation. For a $15 \%$ acceptance energy window at $334 \mathrm{keV}(\sim 50 \mathrm{keV})$ for ${ }^{149} \mathrm{Sm} \mathrm{nSPECT}$ imaging, the Compton count rate from $2.2 \mathrm{MeV}$ photons would be:

$$
\begin{aligned}
\text { Compton counts in acceptance window } & =\frac{\text { Compton } \frac{c}{s}}{k e V} \times \text { window width }(\mathrm{keV}) \\
& =\left(2.1 \times 10^{3} \mathrm{c} \mathrm{s}^{-1} \mathrm{keV}^{-1}\right) \times(50 \mathrm{keV}) \\
& =1.1 \times 10^{5} \mathrm{c} / \mathrm{s}
\end{aligned}
$$

\subsubsection{Patient Equivalent Dose Rate from Neutrons}

The average neutron flux of all tissue layers of the model was $4.5 \times 10^{5} \mathrm{n} \mathrm{cm}^{-2} \mathrm{~s}^{-1}$ (see Table 4, pg.74, for the breakdown by layer), which, using the equivalent dose rates 
per unit neutron flux from Section 3.1.2, results in an average equivalent dose rate between $3.9 \mathrm{mSv} / \mathrm{h}(99 \%$ thermalized spectrum) and $260 \mathrm{mSv} / \mathrm{h}(70 \%$ thermalized spectrum):

Equivalent dose $(99 \%$ thermal $)=\left(8.8 \times 10^{-6} \frac{\mathrm{mSv} / \mathrm{h}}{n \mathrm{~cm}^{-2} \mathrm{~s}^{-1}}\right) \times\left(4.5 \times 10^{5} \mathrm{ncm}^{-2} \mathrm{~s}^{-1}\right)$

$$
=3.9 \mathrm{mSv} / \mathrm{h}
$$

Equivalent dose $(70 \%$ thermal $)=\left(5.8 \times 10^{-4} \frac{\mathrm{msv} / \mathrm{h}}{n \mathrm{~nm}^{-2} \mathrm{~s}^{-1}}\right) \times\left(4.5 \times 10^{5} \mathrm{ncm}^{-2} \mathrm{~s}^{-1}\right)$

$$
=260 \mathrm{mSv} / \mathrm{h}
$$

The equivalent dose rate only applies to tissue exposed to neutrons, which is at most approximately $1 / 4$ of the patient at any time for whole-body imaging. In the case of imaging a small region of the body, the effective dose rate could be significantly lower.

To compare these equivalent dose rate estimates with equivalent doses from ${ }^{99 \mathrm{~m}} \mathrm{Tc}$ bone scans, we will consider the maximum imaging time of 45 minutes (see Section 2.3.3). This yields an average organ equivalent dose range of $2.9 \mathrm{mSv}$ to $195 \mathrm{mSv}$ for nSPECT. For a ${ }^{99 \mathrm{~m}} \mathrm{Tc}$ bone scan, the average equivalent dose range for bone is $57 \mathrm{mSv}$ to $70 \mathrm{mSv}$, for muscle tissue it is $1.4 \mathrm{mSv}$ to $2.1 \mathrm{mSv}$, and for the kidneys it is $5.4 \mathrm{mSv}$ to $8.1 \mathrm{mSv}(19 ; 20)$. The average equivalent dose to bone (the target tissue) may be lower with nSPECT compared to SPECT, but the average equivalent dose to the surrounding tissue (such as muscle) will most likely be higher for nSPECT compared to SPECT. However, if one is performing a bone scan of the hands and wrists to assess arthritis, for 
example, nSPECT may deliver no significant dose to the kidneys, or any other sensitive organs, which is not the case with ${ }^{99 \mathrm{~m}} \mathrm{Tc}$ bone scans.

The decision to use a neutron energy spectrum with a higher or lower thermalization fraction will be based on the available neutron sources and the depth of the target (i.e., bone in this case) in the surrounding tissue. Thermal neutrons are quickly attenuated in highly hydrogenous tissue, so deep targets will benefit from a lower initial neutron thermalization fraction (and thus a higher equivalent dose rate), which would ensure a higher thermal neutron flux at the target depth. In order to reach higher initial thermalization fractions for either shallow or deep tissue, neutrons must pass through larger amounts of moderating material. However, because the neutron source would be isotropic, the neutron flux decreases as the inverse of the distance squared from the source. For example, doubling the distance between the source and the target will reduce the flux in the target by a factor of four. Therefore, the additional separation between source and patient to accommodate additional moderating material will result in a lower neutron flux in the patient. The highest practical neutron thermalization fraction for nSPECT imaging will be limited primarily by the strength of the neutron source.

A mature nSPECT imaging system, will likely dynamically adjust the neutron thermalization fraction and flux to suit each individual imaging procedure (i.e., bone scan, myocardial perfusion, brain perfusion, etc.) and the physical proportions of patients. For example, the thermalization fraction might be high in the extremities such as the hands and forearms, and lower when imaging bone in the thighs. 
In summary, the effective dose range for nSPECT may be in the same range as the effective dose from traditional nuclear medicine procedures for whole-body imaging. In the case of imaging specific regions of the body, nSPECT may deliver a much lower effective dose to patients compared with traditional nuclear medicine.

\subsection{Adding Simulated Hydrogen Noise to the 131I Experiment Results}

Noise was added to images by creating a "noise matrix" based on the $2.2 \mathrm{MeV}$ Compton counts and adding that matrix to the ${ }^{131} \mathrm{I}$ image matrices. The Compton count rate from the end of Section 4.1.3 was multiplied by the acquisition time of an image to obtain the average total number of Compton counts, which was then divided equally between all of the pixels in the noise matrix. In order to achieve the degree of randomness consistent with counting statistics, each pixel of the noise matrix was given a Poisson random number based on the pixel mean Compton counts. The MATLAB function poissrnd $(y)$ was used to generate the random numbers, where $y$ is the pixel mean Compton counts. For example, if the total number of Compton counts for an acquisition was 900 and the image to which the noise matrix is being applied had $3 \times 3$ pixels, then the mean number of Compton counts per pixel and the noise matrix could be:

Mean Compton counts $=\left[\begin{array}{ccc}100 & 100 & 100 \\ 100 & 100 & 100 \\ 100 & 100 & 100\end{array}\right] \quad$ Noise Matrix $=\left[\begin{array}{ccc}103 & 98 & 87 \\ 122 & 110 & 96 \\ 101 & 90 & 100\end{array}\right]$

In creating the noise matrices, two different scenarios were considered: one scenario corresponding to a natural abundance samarium tracer and the other 
corresponding to a $99+\%$ enriched ${ }^{149} \mathrm{Sm}$ tracer. For the natural abundance $\mathrm{Sm}$ tracer scenario, a 2-minute ${ }^{131}$ I image was summed with a noise matrix containing two minutes of Compton counts, as defined in Section 4.1.3. For the $99+\%$ enriched ${ }^{149} \mathrm{Sm}$ tracer scenario, a 2-minute ${ }^{131} \mathrm{I}$ image would be produced in 17 seconds, because there is 7.25 times more ${ }^{149} \mathrm{Sm}$ per mole of enriched tracer (natural abundance of ${ }^{149} \mathrm{Sm}$ is $13.8 \%$ ). Therefore, the 2 -minute ${ }^{131} \mathrm{I}$ image was summed with a noise matrix containing 17 seconds of Compton counts.

When introducing the tissue model from Section 4.1.1, the additional signal attenuation of the tissue (i.e., water) must be considered. The $6 \mathrm{~cm}$ of water between the plane of the signal activity and the plane of the detector results in $49 \%$ attenuation at 364 $\mathrm{keV}$, compared to $21 \%$ by the $2 \mathrm{~cm}$ thick acrylic ${ }^{131} \mathrm{I}$ phantom top (see Appendix E for calculations). The image acquisition times for both natural and enriched tracer types and their noise matrices must be multiplied by approximately 1.5 to account for the attenuation difference of $28 \%$ (see Appendix E for calculations). The acquisition time to produce the same number of signal counts as a 2-minute ${ }^{131} \mathrm{I}$ image becomes approximately 3.1 minutes (i.e., $\sim 1.5 \times 2$ minutes) for the natural abundance tracer, and 26 seconds (i.e., $\sim 1.5 \times 17$ seconds) for the enriched tracer. For both tracer scenarios, 90 noise matrices were created to correspond with the 902 -minute ${ }^{131}$ I phantom images. An example of a single 2 -minute ${ }^{131} \mathrm{I}$ image (128x128 matrix size) summed with a single noise matrix from each Sm tracer type is shown in Figure 22. 

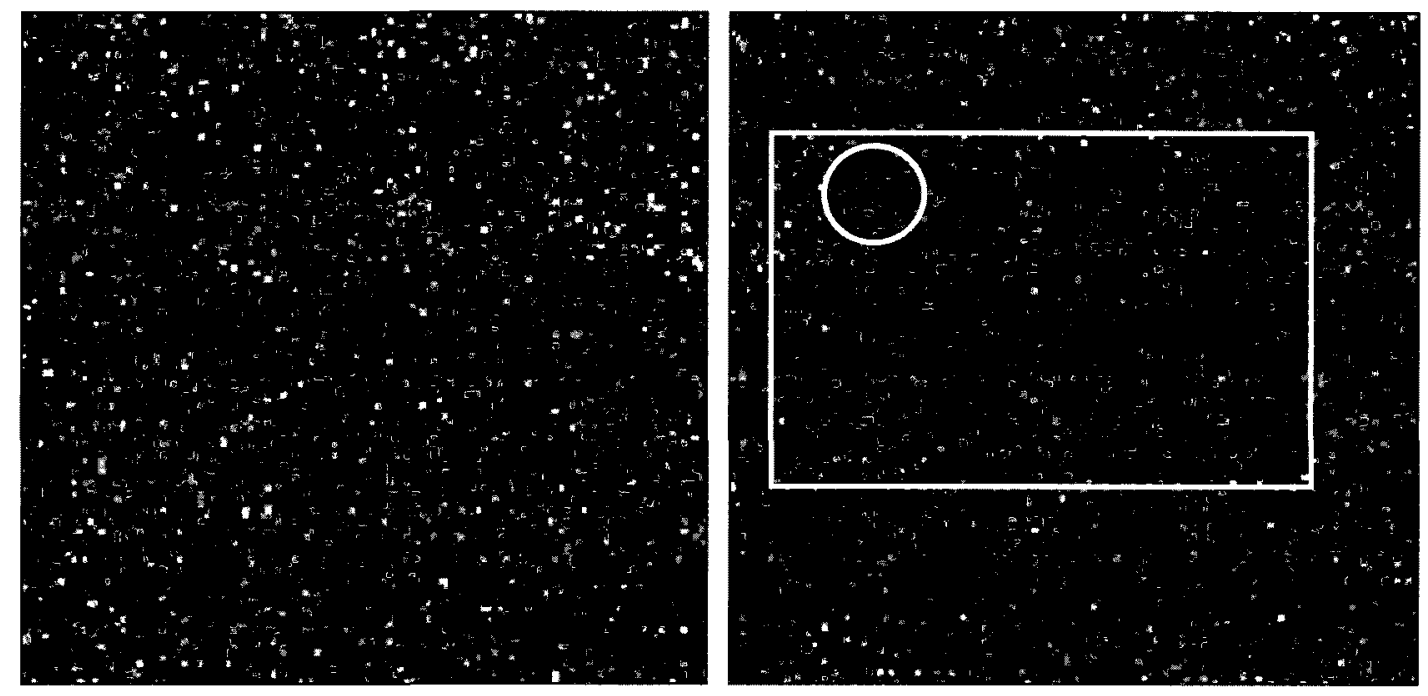

Figure $22-128 \times 128$ pixel images of a 2-minute ${ }^{131} \mathrm{I}$ image combined with the $2.2 \mathrm{MeV}$ Compton counts (i.e., noise matrix) from the two Sm tracer scenarios. The signal is visibly hidden for the natural abundance tracer scenario (left). A few of the larger and more intense characteristics of the ${ }^{131}$ I image can be seen in the enriched tracer scenario (right), since there are fewer Compton counts.

\subsection{Analysis of Images}

There are three types of images to be analyzed:

- the ${ }^{131} I$ images

- the ${ }^{131} \mathrm{I}$ images with simulated Compton noise, corresponding to neutron activation of a natural abundance Sm tracer surrounded by tissue

- and the ${ }^{131} \mathrm{I}$ images with simulated Compton noise, corresponding to neutron activation of a $99+\%$ enriched ${ }^{149} \mathrm{Sm}$ tracer surrounded by tissue 
The analysis method consists of calculating the $\mathrm{SNR}_{\text {Rose }}$ for each of the four lesions in the highest contrast category (corresponding to EDTMP bone uptake rates) in addition to subjective visual evaluation. In practice, a real nSPECT image would be processed with triple-energy-window background and scatter-correction (56) and some form of filtering in order to reduce the degrading effect of the Compton background produced from hydrogen activation. Therefore, the experimental images were processed with a simulation of triple-energy-window background scatter-correction and a low-pass filter was applied. Subjective visual assessment was used to establish a threshold $\mathrm{SNR}_{\text {Rose }}$ value that can be considered acceptable. The $\mathrm{SNR}_{\mathrm{Rose}}$ values were then used as an objective method of measuring image quality relative to the threshold.

Each of the three image types was evaluated at 90 time intervals, corresponding to the $90{ }^{131} \mathrm{I}$ images from the ${ }^{131} \mathrm{I}$ Experiment. The noisy ${ }^{131} \mathrm{I}$ images simulating the natural abundance $\mathrm{Sm}$ tracer were evaluated at 3.1 minute intervals ( 2 minute ${ }^{131} \mathrm{I}$ acquisition multiplied by $\sim 1.5$ for simulated signal attenuation) according to the analysis presented in Section 4.2. The noisy ${ }^{131} \mathrm{I}$ images simulating the $99+\%$ enriched ${ }^{149} \mathrm{Sm}$ tracer were evaluated at 26 second intervals ( 2 minute ${ }^{131} \mathrm{I}$ acquisition multiplied by $\sim 1.5$ for simulated signal attenuation and divided by 7.25 for increased activity due to enrichment) according to the analysis presented in Section 4.2. The unaltered ${ }^{131} \mathrm{I}$ images were evaluated on both timescales (i.e., for both tracer types), in order to provide a reference standard for both tracer scenarios. 
The $\mathrm{SNR}_{\mathrm{Rose}}$ was calculated for each lesion using a modified form of equation 1:

$$
S N R_{\text {Rose }}=\frac{\sum_{i=1}^{K}\left(c_{i}-B_{A v g}\right)}{\sqrt{\sum_{i=1}^{K} c_{i}}}=\frac{\sum_{i=1}^{K} c_{i}-K B_{A v g}}{\sqrt{\sum_{i=1}^{K} c_{i}}}
$$

where $K$ is the total number of pixels in the lesion area, $\mathrm{c}_{l}$ is the total number of counts in pixel $i$, and $B_{A v g}$ is the average number of background counts per pixel. $B_{A v g}$ was calculated from a large healthy-tissue region of the phantom where there are no lesions, as shown, for example, by the red rectangle in Figure 23.

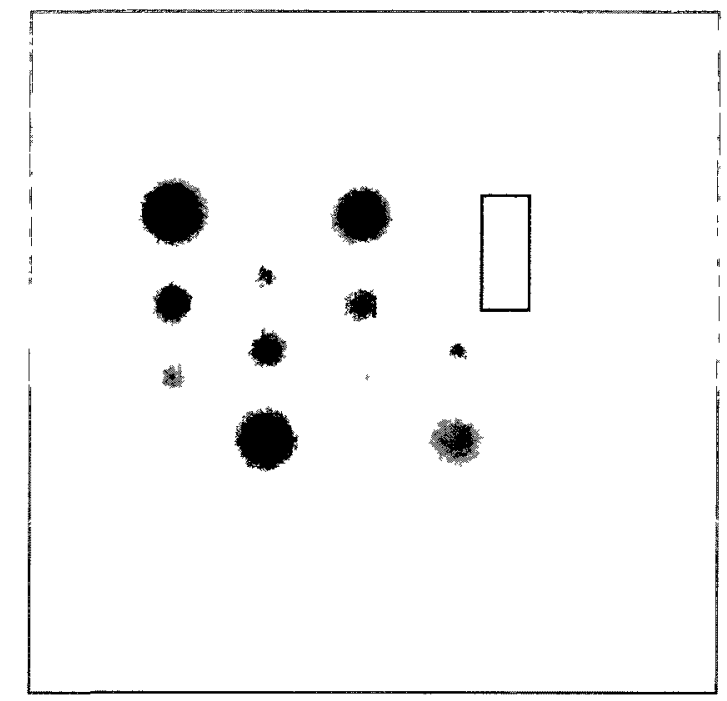

Figure 23 - A 180 -minute image of the ${ }^{131}$ I phantom showing the region used to calculate $B_{A v g}$, outlined by the red rectangle. 
The variance of the $\mathrm{SNR}_{\text {Rose }}$ (equation 13) can be expressed as:

$$
\begin{aligned}
\operatorname{Var}(S N R) & =\left(\frac{\partial S N R}{\partial\left(\sqrt{\sum_{l=1}^{K} c_{l}}\right)}\right)^{2} \operatorname{Var}\left(\sqrt{\sum_{l=1}^{K} c_{l}}\right)+\left(\frac{\partial S N R}{\partial\left(K B_{A v g}\right)}\right)^{2} \operatorname{Var}\left(K B_{A v g}\right) \\
& =\frac{1}{4}\left(1+\frac{K B_{A v g}}{\sum_{i=1}^{K} c_{i}}\right)^{2}+\frac{K^{2} \operatorname{Var}\left(B_{A v g}\right)}{\sum_{i=1}^{K} c_{i}}
\end{aligned}
$$

where the nomenclature is consistent with equation 13 , and $\operatorname{Var}\left(B_{\text {avg }}\right)$ is the experimental variance of the mean, $B_{\text {avg }}$

Simulated triple-energy-window background- and scatter-correction for image enhancement was accomplished by subtracting the mean Compton counts per pixel from the noisy images. In practice, the background- and scatter-correction method involves placing an additional energy window on either side of the acceptance energy window. The counts in those secondary windows are then used to estimate the Compton background (56). The background is subtracted from the counts in the acceptance window, enhancing image contrast and allowing for more accurate quantitative analysis (56). In the case of this thesis analysis, the mean number of Compton background counts is known precisely because the Compton background was artificially added to the images. However, after subtracting the precise mean background from the images, the statistical variation of the background Compton counts remains, so a complete restoration of the image does not occur. 
Images were also low-pass filtered to reduce the effects of white noise created by the 2.2 MeV Compton counts. The filter was a commonly-used two-dimensional filter known as a nine-point binomial spread function, shown in equation 15, which is applied in the spatial domain (33). The filter function is convolved with the signal to produce the filtered image. An example with a one-dimensional signal and filter is shown in Figure 24. Ideally, a restoration filter, such as a Metz or Wiener filter, could be implemented to recover a maximum amount of information from the noisy image (33). It is premature to design and implement these advanced filters given the objective of the ${ }^{131}$ I Experiment, which was to determine if ${ }^{131}$ I could produce reasonable images at an activity comparable to ${ }^{149} \mathrm{Sm}$ PGNA at reasonable chemical and neutron doses.

$$
\omega=\left[\begin{array}{lll}
1 & 2 & 1 \\
2 & 4 & 2 \\
1 & 2 & 1
\end{array}\right]
$$

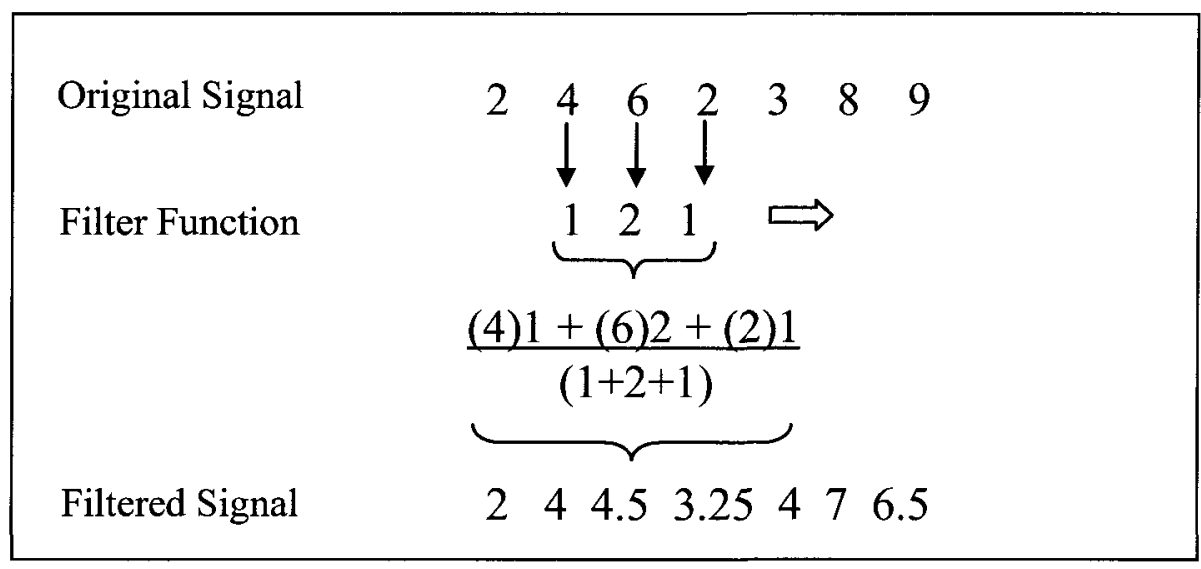

Figure 24 - An example of one-dimensional filtering with a three-point binomial filter. The center of the filter is moved over each element of the original signal, and the filtered signal is produced from the normalized weighted sum of the filter values multiplied by the original signal values. (33) 


\subsubsection{Compton-free Images (Unaltered 131I Phantom Images)}

A plot of the $\mathrm{SNR}_{\text {Rose }}$ for a range of image acquisition times and a $128 \times 128$ matrix can be found in Figure 25. A filtered ${ }^{131}$ I image corresponding to an equivalent 10 -minute acquisition time for both natural abundance and enriched Sm tracers can be found in Figure 26. In the natural abundance $\mathrm{Sm}$ tracer image, the $1 \mathrm{~cm}$ lesion, with a $\mathrm{SNR}=8$, is faintly visible above the background. Therefore, we can establish that, for the images produced in this work, lesions with a SNR $>8$ are visible. In Figure 25, the $1 \mathrm{~cm}$ lesions are visible after imaging times of 10 minutes or less. However, the $0.5 \mathrm{~cm}$ lesion only becomes visible after 25 minutes for the enriched tracer and 180 minutes for the natural abundance tracer. 
Simulated lesion SNR for I-131 Images

Imaging Time (min), natural abundance Sm tracer

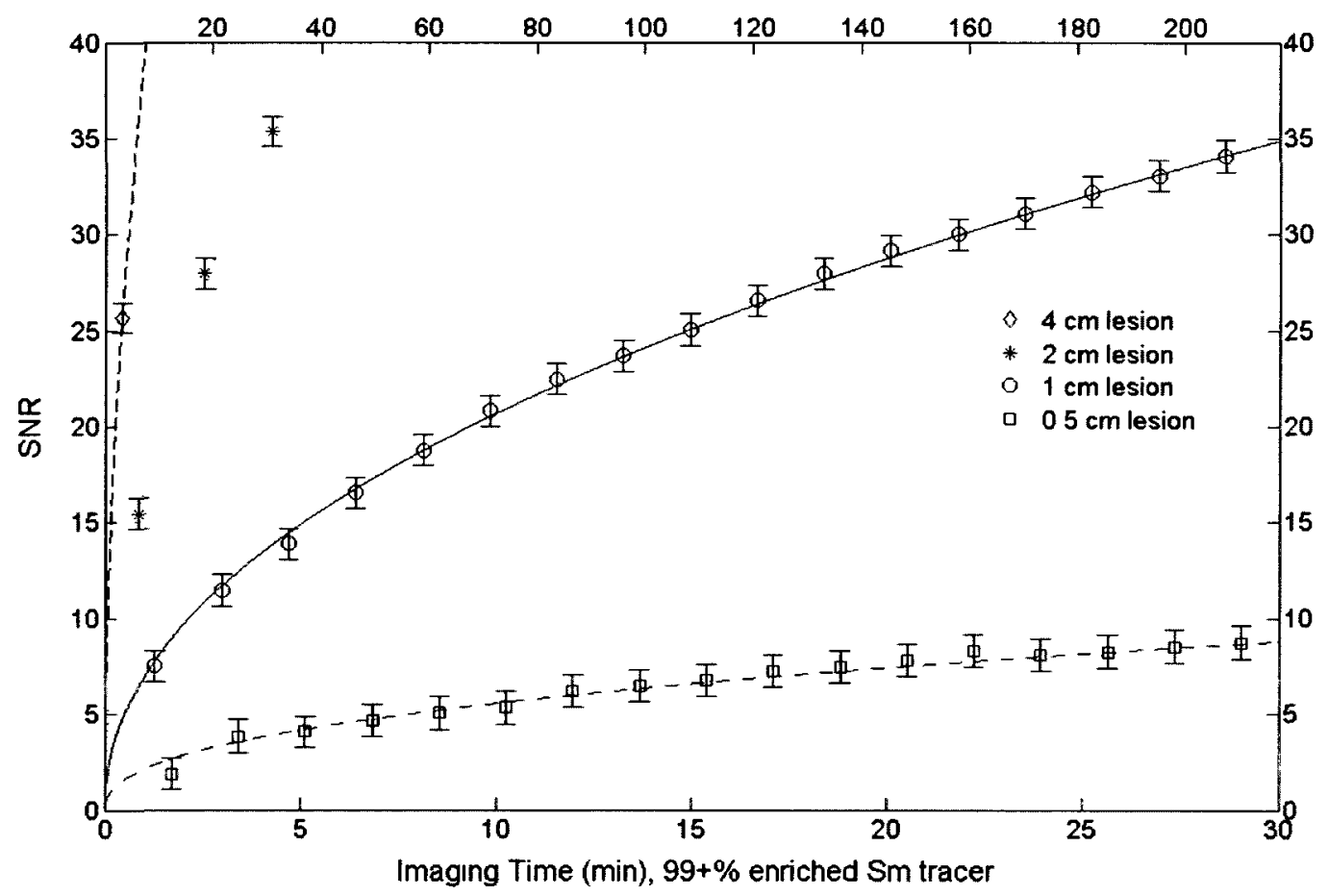

Figure 25 - A plot of lesion $S_{\text {SNRe }}$ against image acquisition time for the highest contrast group. Error bars represent \pm one standard deviation (i.e., $69 \%$ confidence interval). Every fourth data point is plotted in each series to reduce clutter in the plot. This is the reference standard for nSPECT bone imaging, because it contains no Compton counts from $2.2 \mathrm{MeV}$ hydrogen PGNA. Time-scales are provided for both natural abundance (top) and enriched (bottom) tracers.
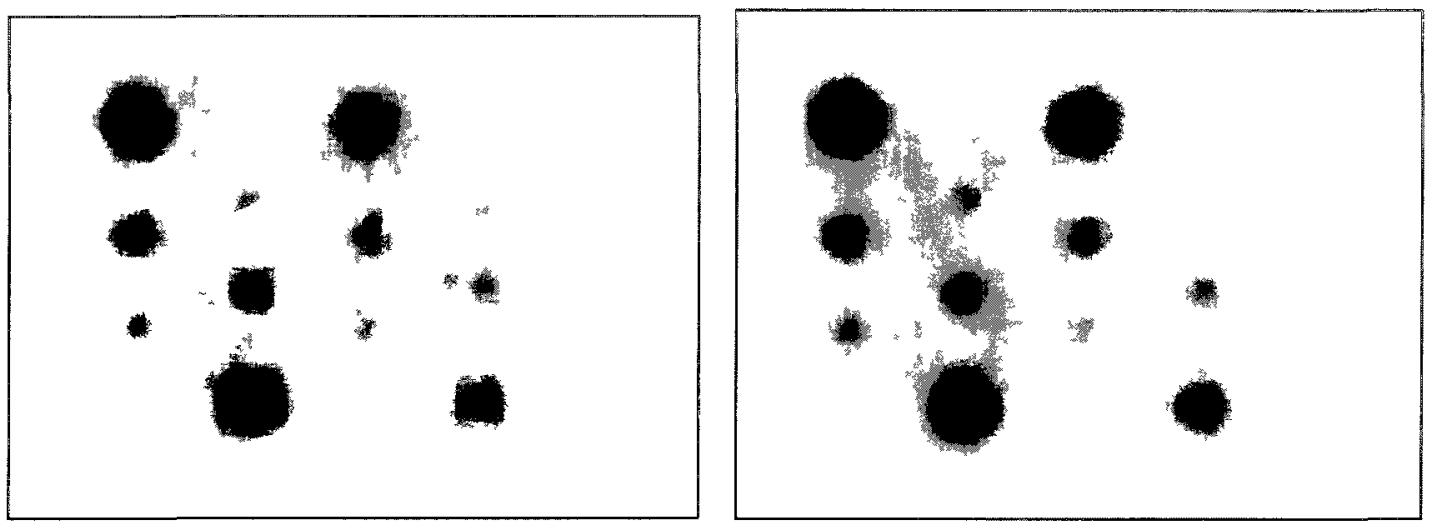

Figure 26 - Filtered ${ }^{131}$ I images simulating 10-minute acquisitions for natural abundance (left) and enriched (right) Sm tracers. The $1 \mathrm{~cm}$ lesion (third lesion down on the far left of the images) has a SNR of approximately 8 in the left image and approximately 20 in the right image. 


\subsubsection{Natural Abundance Samarium target}

A plot of the $\mathrm{SNR}_{\text {Rose }}$ for a range of image acquisition times and a $128 \times 128$ matrix size can be found in Figure 27. Filtered and scatter-subtracted ${ }^{131} \mathrm{I}$ images corresponding to 10 -minute and 30-minute acquisition times for a natural abundance $\mathrm{Sm}$ tracer can be found in Figure 28. No lesions are visible in the 10-minute image and only the $4 \mathrm{~cm}$ lesion (top lesion on the far left of the image) is visible in the 30-minute image. Therefore, natural $\mathrm{Sm}$ is not an acceptable target based on the parameters of the simulation presented in this thesis. Increasing the tracer concentration would improve image quality, however, the large increases in tracer concentration needed to achieve acceptable image quality may begin to cause undesirable medical side-effects for the patient (see Section 2.5.2). Alternatively, if the Compton counts could be identified and rejected during acquisition, by using a Compton suppression detector (13), for example, natural abundance Sm tracers may yet be viable for imaging, as seen in Figure 26. 
Simulated lesion SNR for $1-131$ images with artificial noise:

natural abundance Sm tracer

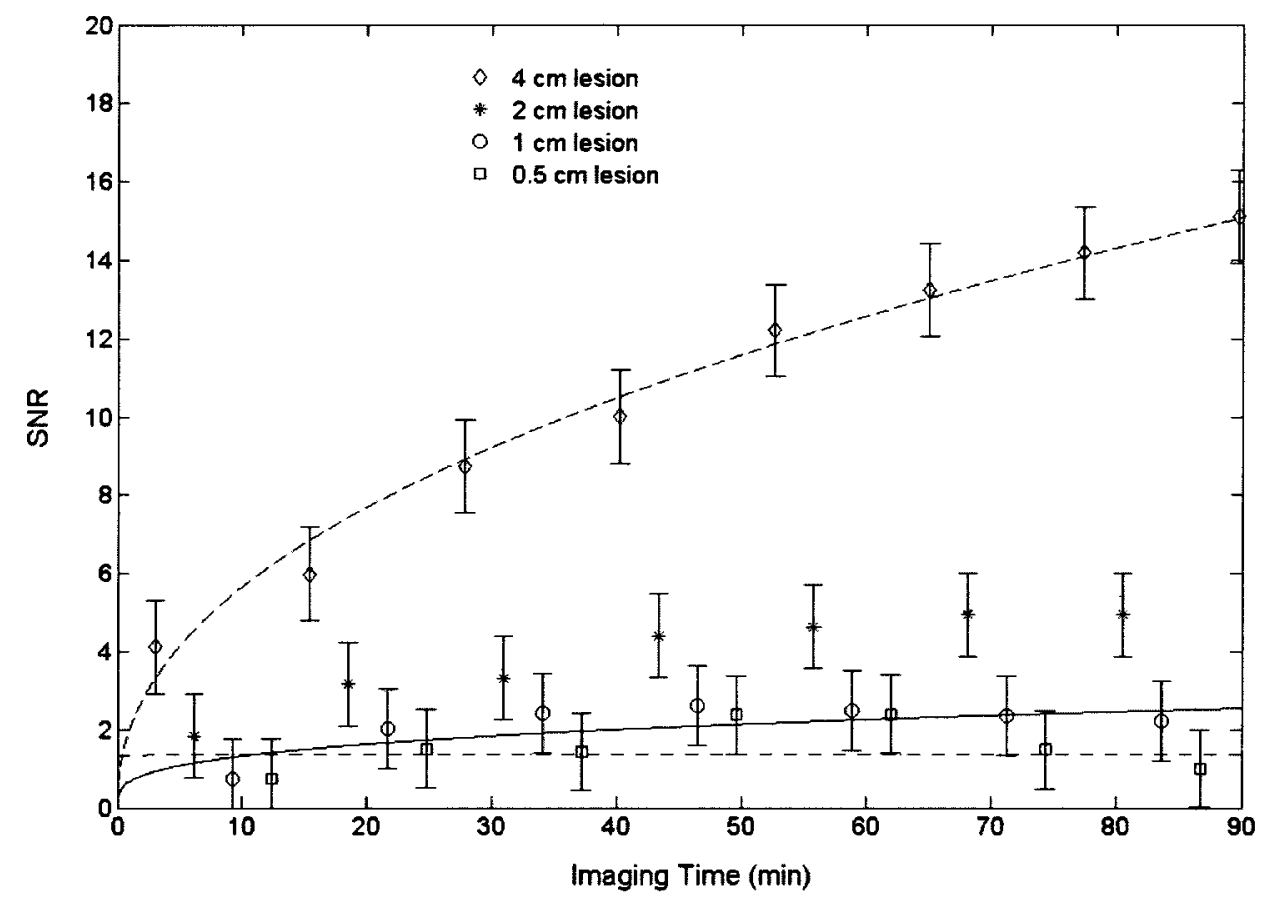

Figure 27 - A plot of lesion SNR $_{\text {Rose }}$ against image acquisition time for the highest contrast group. Error bars represent \pm one standard deviation (i.e., $69 \%$ confidence interval). Every fourth data point is plotted in each series to reduce clutter in the plot. These data were produced based on a natural abundance $\mathrm{Sm}$ tracer.
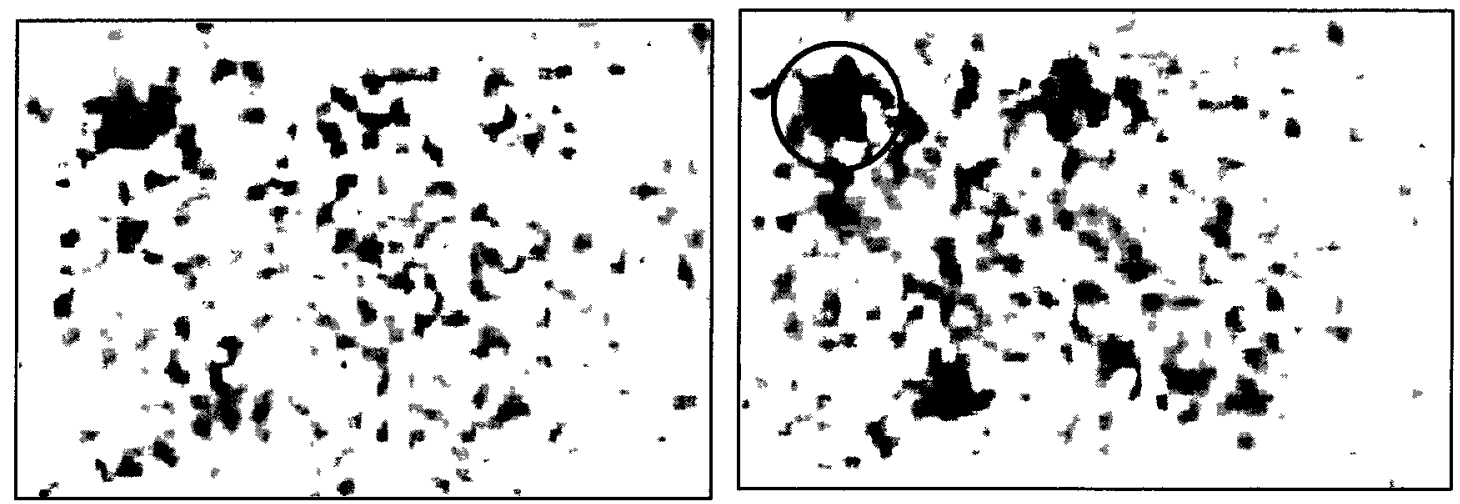

Figure 28 - Scatter subtracted and filtered images of 10-minute (left) and 30-minute (right) acquisitions for a natural abundance tracer. None of the lesions are visible in the 10-minute image. Only the $4 \mathrm{~cm}$ lesion is visible in the 30 minute image. 


\subsubsection{Enriched ${ }^{149} \mathrm{Sm}$ target}

Predictions of the $\mathrm{SNR}_{\text {Rose }}$ for a range of image acquisition times and a $128 \times 128$ matrix are shown in Figure 29. Filtered and scatter-subtracted ${ }^{131} \mathrm{I}$ images corresponding to 10 -minute and 30-minute acquisition times for a $99+\%$ enriched $\mathrm{Sm}$ tracer can be found in Figure 30. The $4 \mathrm{~cm}$ and $2 \mathrm{~cm}$ lesions (top two lesions on the far left of the image), with SNR $=34$ and 11 respectively, are visible in the 10-minute image. The 4 $\mathrm{cm}, 2 \mathrm{~cm}$, and $1 \mathrm{~cm}$ lesions (top three lesions on the far left of the image), with SNR = 58,23 , and 7.5 respectively, are visible in the 30 -minute image. Therefore, the highly enriched ${ }^{149} \mathrm{Sm}$ tracer might be used to identify lesions as small as $2 \mathrm{~cm}$ for full-body imaging, and as small as $1 \mathrm{~cm}$ if a single spot view of a region of the body is acquired.

While these are promising results for a preliminary experiment, there are some concerns with using a highly enriched tracer. For the conditions described in Section 3.4.3, a natural abundance tracer in healthy bone tissue would not experience significant neutron flux depression. However, the $99+\%$ enriched ${ }^{149} \mathrm{Sm}$ tracer would be 7.25 times more concentrated than the natural abundance Sm tracer and it may cause neutron flux depression equivalent in magnitude to that experienced by the $500 \mathrm{ppm}$ case in the McMaster Experiment (Section 3.1.2). The flux depression could result in reduced lesion signal, which would make it more difficult to identify small lesions, such as the $1 \mathrm{~cm}$ and $0.5 \mathrm{~cm}$ simulated lesions in the ${ }^{131} \mathrm{I}$ phantom. 
It is unclear how significant this flux depression would be for nSPECT bone imaging, since the geometry of the McMaster phantom differs from the geometry of the various bones and potential lesion shapes and sizes in the body. Additionally, the tracer distribution, which was homogenous in the McMaster phantom, would be different for nSPECT, where it would be primarily concentrated on the bone surface. The most likely effect of neutron flux depression at these levels would be a reduction in the effective contrast between lesions and healthy tissue, to the point where smaller lesions on the order of $1 \mathrm{~cm}$ may be indistinguishable from the surrounding tissue.

Neutron flux attenuation is a well-understood phenomenon and there are established methods of correcting for its effects (13). In PGNAA, sample calibration standards are used to develop correction factors which are applied to measurements (13). For complex samples and geometries, Monte Carlo software, such as MCNP, can be used to determine the impact of neutron flux attenuation on the measurements; this information can be used to develop more sophisticated correction factors (13). These methods could be applied to nSPECT. 
Simulated lesıon SNR for I-131 images with artıficial noise

$99+\%$ enriched $\mathrm{Sm}$ tracer

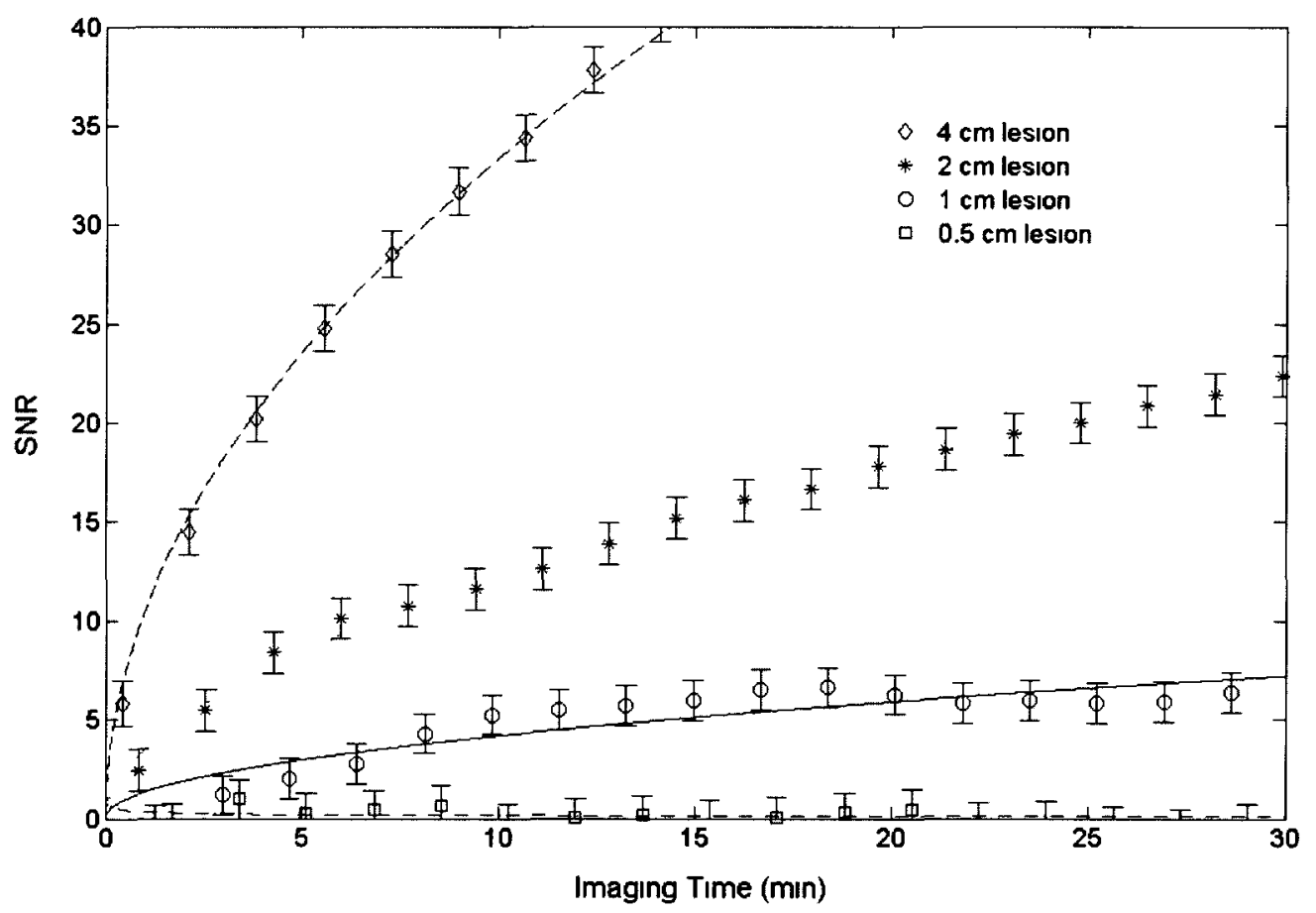

Figure 29 - A plot of lesion SNR $_{\text {Rose }}$ against image acquisition time for the highest contrast group. Error bars represent \pm one standard deviation (i.e., 69\% confidence interval). Every fourth data point is plotted in each series to reduce clutter in the plot. These data represent an enriched ${ }^{149} \mathrm{Sm}$ tracer.
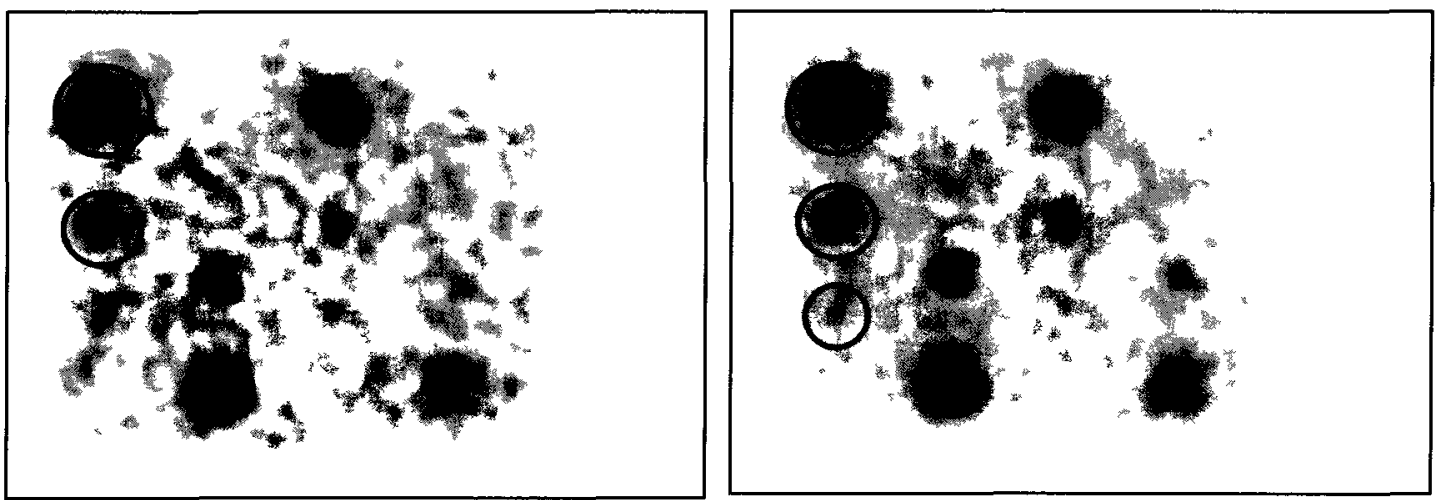

Figure 30 - Scatter subtracted and filtered simulated images of 10-minute (left) and 30minute (right) acquisitions for an enriched tracer. The $4 \mathrm{~cm}$ and $2 \mathrm{~cm}$ lesions are visible in the 10-minute image. Those lesions are also easily visible in the 30 -minute image. The $1 \mathrm{~cm}$ lesion is barely visible in the 30minute image. 


\section{CHAPTER 5:RECOMMENDATIONS TO IMPROVE IMAGE QUALITY}

There are a few ways that image quality could be improved for the images presented in Section 4.3, using methods and features that are currently available in modern SPECT imaging systems. The first and simplest method would be to reduce the width of the acceptance energy window for data acquisition. The $55 \mathrm{keV}$ acceptance energy window width used in the ${ }^{131}$ I Experiment is designed to optimize the SNR when counting with a low Compton background, as is the case with radioisotope-based SPECT imaging. However, radioisotope-based SPECT does not have the same level of Compton background that is expected to be present with nSPECT. A $35 \mathrm{keV}$ acceptance energy window provides the optimal SNR for imaging with a high Compton background (see Figure 31); the time required to reach $\mathrm{SNR}=8$, which is the threshold of visibility for a lesion as defined in Section 4.3, is estimated to decrease by $9 \%$ for all lesions ${ }^{8}$.

Another way to improve image quality is to take advantage of the secondary photon peak from ${ }^{149} \mathrm{Sm}$ PGNA (see Table 2 in Section 2.4.3). The $440 \mathrm{keV}$ photon has more than one-half the intensity of the primary $334 \mathrm{keV}$ photon. Using this additional photon may lead to a reduction of resolution, due to an increase in collimator septa edge

\footnotetext{
${ }^{8}$ The average time required to achieve a given SNR can be calculated by rearranging a modified version of equation 1 and solving for time: $S N R=\frac{(\text { signal } c / s)(\text { time })}{\sqrt{(\text { totalc/s)(time })}}$. The count rates from the ${ }^{131}$ I Experiment and Compton noise simulation were used in a numerical calculation that identified $35 \mathrm{keV}$ as the optimal energy window. For all of the simulated lesions at a 15:1 uptake ratio, a SNR $=8$ was achieved $9 \%$ faster when using a $35 \mathrm{keV}$ window instead of a $50 \mathrm{keV}$ window.
} 
penetration as a result of its higher energy. However, the increase in lesion SNR from the additional signal counts at $440 \mathrm{keV}$ may provide a net benefit.

\section{Gamma Camera Count Rate per keV}

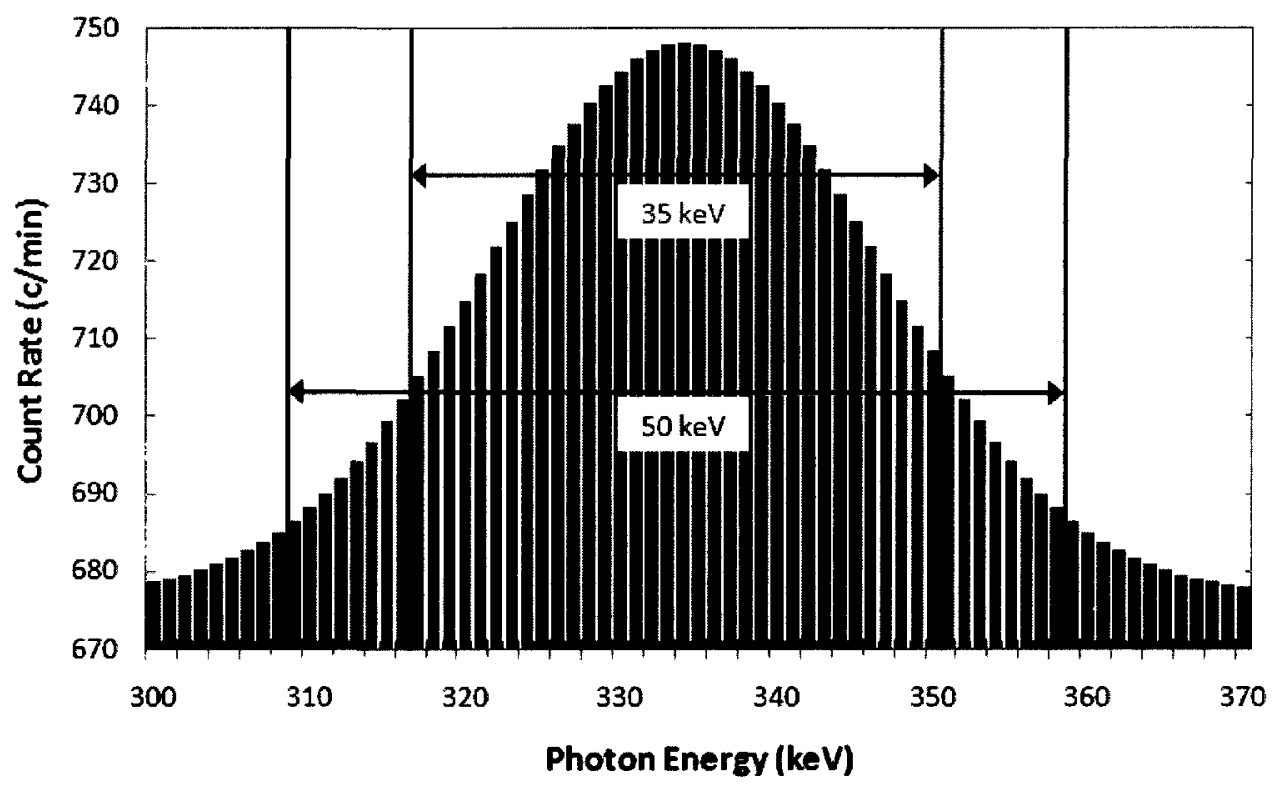

Figure 31 - A plot of the count rate per unit $\mathrm{keV}$ of a $4 \mathrm{~cm}$ lesion with an enriched ${ }^{149} \mathrm{Sm}$ tracer and Compton counts from $2.2 \mathrm{MeV}$ gamma rays. The $50 \mathrm{keV}$ $(15 \%$ at $334 \mathrm{keV})$ energy window used in this experiment is shown. The 35 $\mathrm{keV}$ energy window, which provides an optimal SNR for this scenario, is also shown.

Significant increases in affordable computing power over the last decade have encouraged the development of some advanced SPECT image filtering methods. In addition to powerful restoration filters like the Metz and Wiener filter, advanced adaptive filters such as the PIXON and SHINE methods are available (33). The SHINE filtering method uses an image compression-reconstruction approach that produces an image that 
is indistinguishable from a raw image with double the number of counts (57). The PIXON filter uses a locally adaptive Gaussian smoothing algorithm which, when the filtered image is blended with the original image, can significantly reduce false-positive rates, and increase true-positive rates, in lesion identification (58). The PIXON technique has been adopted by ImageRecon LLC and Siemens Medical Solutions, Inc., who design and build SPECT systems (58). Employing any of these advanced filters in place of the basic low-pass filter from Section 4.3 would enhance image quality. 


\section{CHAPTER 6: RECOMMENDATIONS FOR FUTURE EXPERIMENTS}

From the analysis in 4.3 , it is apparent that Compton noise from hydrogen PGNA results in a significant reduction in image quality, particularly when using a natural abundance Sm tracer. Therefore, any future experiment or simulation should seek to improve the current understanding of the impact that $2.2 \mathrm{MeV}$ gamma rays have on images acquired with a SPECT imaging system. The analysis of hydrogen noise provided in this report was based on simulation data alone and did not include collimator effects. This future experiment would require:

- A gamma camera with a High Energy collimator and all the necessary data acquisition electronics, ideally capable of using multiple energy windows

- A neutron source, likely either a radioisotope or accelerator source

- A water phantom with a simple geometry. This will provide a source of $2.2 \mathrm{MeV}$ photons. The simple geometry will make the test setup easy to model

- Monte Carlo software capable of neutron transport calculations, such as MCNP. This will be used to model the experimental setup and to calculate the neutron flux spatial distribution

The water phantom would be exposed to neutrons, generating $2.2 \mathrm{MeV}$ gamma rays, and the gamma camera would measure the Compton background within the acceptance energy window. The data from the Monte Carlo simulation would be used to scale the measurements (i.e., $\mathrm{X}$ neutron flux in $\mathrm{Y}$ amount of water produces $\mathrm{Z}$ Compton 
counts). If the water phantom were constructed to be similar to the ${ }^{131} \mathrm{I}$ phantom from Section 3.4.2, the Compton images could be scaled appropriately and added to the ${ }^{131} I$ phantom images, providing a better estimate of an nSPECT bone image.

Another important parameter that should be addressed in a future experiment or simulation is the patient radiation dose rate from neutrons. The analysis in Section 4.1.4 suggested an equivalent dose rate between $3.9 \mathrm{mSv} / \mathrm{h}(99 \%$ thermalized spectrum) and $260 \mathrm{mSv} / \mathrm{h}(70 \%$ thermalized spectrum), which is a wide range. The low end of that range is feasible for clinical applications, but the high-end exceeds modern SPECT equivalent doses and is unlikely to be considered acceptable (see Section 4.1.4). Therefore, an experiment should be conducted to determine the range of feasible neutron flux thermalization fractions and patient doses. The following factors should be considered:

- Likely neutron sources to be used in a commercial nSPECT system. For example, a research reactor might not be practical in a typical nuclear medicine department because of its large size, relatively high cost, and low social acceptability (59)

- Neutron moderating materials and configurations that achieve the approximate required neutron flux in the target tissue

- Target tissue geometry and location in the patient; an anthropomorphic tissue model should be used 
- The relative orientation between the Gamma Camera(s) and the neutron source (i.e., the $180^{\circ}$ separation assumed in Section 4.1 .1 , or possibly some other configuration)

The neutron source is likely to be the most expensive and challenging (in terms of regulatory requirements) item to acquire and operate, assuming a licensed facility is not currently available. It may be more feasible to begin with a Monte Carlo simulation prior to conducting an experiment. The simulation results should be sufficiently accurate for a feasibility study regarding neutron flux thermalization and dose rates. Depending on the results of the simulation, an experiment may be justified in order to confirm the simulation findings. 


\section{CHAPTER 7: CONCLUSIONS}

The concept of nSPECT has been introduced for the application of nuclear medicine planar bone imaging. The goal of this work was to conduct a preliminary assessment of the feasibility of the nSPECT method. To this end, patient tracer dose, radiation equivalent dose rates, and image quality were estimated using experimental data.

Planar images of a phantom containing ${ }^{131} \mathrm{I}$ as a proxy for neutron-activated ${ }^{149} \mathrm{Sm}$ tracers were analyzed. The analysis showed that simulated lesions as small as $1 \mathrm{~cm}$ in diameter can be detected for an imaging time under 10 minutes. The images were then degraded by adding simulated background counts from high-energy Compton interactions, which would be produced from concomitant hydrogen neutron activation in the body. As a result, lesion visibility for the natural abundance Sm tracer would be reduced so that only the $4 \mathrm{~cm}$ lesion could be identified, and only after a 30 -mintue imaging time. For the enriched ${ }^{149} \mathrm{Sm}$ tracer, $4 \mathrm{~cm}$ and $2 \mathrm{~cm}$ lesions would still be visible for an imaging time of 10 minutes; however, the $1 \mathrm{~cm}$ lesion only became visible after a 30-minute imaging time. For enriched ${ }^{149} \mathrm{Sm}$ tracers, neutron flux attenuation may be significant, which could reduce the signal from the lesions and lead to longer imaging times. The results listed above are accompanied by the following simulated parameters:

- Average Maxwellian neutron flux (at $298 \mathrm{~K}$ ) of $4.0 \times 10^{5} \mathrm{n} \mathrm{cm}^{-2} \mathrm{~s}^{-1}$ in the target

- Sm-EDTMP tracer concentration of $125 \mathrm{ppm}(\mathrm{g} / \mathrm{g})$ in the target (i.e., bone) 
- Equivalent dose rate from neutrons between $3.9 \mathrm{mSv} / \mathrm{h}(99 \%$ thermalized flux $)$ to $260 \mathrm{mSv} / \mathrm{h}$ ( $70 \%$ thermalized flux). The effective dose is dependent on the quantity and type of tissue exposed to the neutron beam, as well as the exposure time

The ${ }^{131} \mathrm{I}$ Experiment and the accompanying simulation and analysis have demonstrated that the Compton background produced by hydrogen activation in the body should have a significant impact on image quality. Therefore, future experiments should address this by obtaining experimental data on high-energy photon interactions with a collimated gamma camera to replace the simulation-based estimates from this work. Additionally, the equivalent radiation dose was shown to be potentially within the same range as the equivalent radiation dose from current ${ }^{99 \mathrm{~m}}$ Tc-based bone SPECT. However, the estimated range of the equivalent radiation dose rate from neutrons is too broad, due to the limited amount of data available, and future simulation or experimental work should narrow the range of this estimate.

Multiple methods were identified for improving image quality beyond what was presented in this thesis, including modern image filters, multi-peak analysis and acceptance energy window resizing. All of these techniques are currently employed in SPECT and planar nuclear medicine imaging.

The ${ }^{131}$ I Experiment and subsequent analysis have shown that nSPECT bone imaging could meet or exceed the requirements of current nuclear medicine bone imaging. In addition to being immune to radioisotope supply issues, nSPECT offers new 
possibilities for localizing tracer activity and radiation dose and enhancing patient and operator safety. Considering the results presented in this thesis and the size of the market in which nSPECT could be competitive, further research and development of nSPECT imaging is warranted. 
APPENDIX A - CALCULATIONS FOR EDTMP ORAL CONSUMPTION

\begin{tabular}{|rrrr|}
\hline Table of Constants & & & \\
\hline Molar mass of EDTMP $\left(\mathrm{C}_{6} \mathrm{H}_{12} \mathrm{O}_{12} \mathrm{~N}_{2} \mathrm{P}_{4} \mathrm{Na}_{5}\right)=$ & 545.9 & $\mathrm{~g} / \mathrm{mol}$ & Ref: (60) \\
Molar mass of $\mathrm{Sm}-149=$ & 148.917 & $\mathrm{~g} / \mathrm{mol}$ & Ref: (13) \\
Patient mass $=$ & 70 & $\mathrm{~kg}$ & \\
EDTMP Bone uptake (Oral) $=$ & $3.2 \%$ & & Ref: (46) \\
${ }^{149}$ Sm-EDTMP biological half-life $=$ & 21.7 & days & Ref: (22) \\
\hline
\end{tabular}

$$
\begin{aligned}
{ }^{149} \text { SmEDTMP transformation constant, } \quad \lambda & =\frac{\ln (2)}{{ }^{149} \text { SmEDTMP bio halflife }} \\
& =\frac{\ln (2)}{21.7 \text { days }}=0.032 \text { days }^{-1}
\end{aligned}
$$

Sample calculations for $500 \mathrm{mg} / \mathrm{kg} /$ day regimen:

$$
\begin{aligned}
& \operatorname{Sm}(x)=\text { mass of }{ }^{149} \mathrm{Sm} \text { accumulated in bone at the end of day } x \\
& \operatorname{Sm}(x)=\underbrace{S m(x-1) e^{-\lambda(1 \text { day })}}+\underbrace{\frac{0.5 \frac{\text { gof EDTMP }}{\mathbf{k g}} \times \text { Patient mass in kg }}{\mathrm{M}_{\text {EDTMP }}} \times \mathrm{M}_{\mathrm{Sm}-149} \times \text { Bone uptake }} \\
& \text { Accounts for the removal of } \\
& \text { accumulated }{ }^{149} \text { Sm-EDTMP } \\
& \text { Contribution by the latest pharmaceutical } \\
& \text { dose administered to the patient on day } x \\
& \text { according to the biological half-life } \\
& \operatorname{Sm}(1)=[0]+\left[\frac{0.500 \frac{\mathrm{g} \mathrm{of} \mathrm{EDTMP}}{\mathrm{kg}} \times 70 \mathrm{~kg}}{545.9 \frac{\mathrm{g} \mathrm{of} \mathrm{EDTMP}}{\mathrm{mol}}} \times 148.9 \frac{\mathrm{g} \mathrm{of} \mathrm{Sm}}{\mathrm{mol}} \times 0.032\right] \\
& =0.30 \mathrm{~g} \text { of } \mathrm{Sm}
\end{aligned}
$$




$$
\begin{aligned}
\operatorname{Sm}(2) & =\left[\operatorname{Sm}(1) \times \mathrm{e}^{-0.032}\right]+\left[\frac{0.500 \frac{\mathrm{g} \text { of EDTMP }}{\mathrm{kg}} \times 70 \mathrm{~kg}}{545.9 \frac{\mathrm{g} \text { of EDTMP }}{\mathrm{mol}}} \times 148.9 \frac{\mathrm{g} \mathrm{of} \mathrm{Sm}}{\mathrm{mol}} \times 0.032\right] \\
& =0.29 \mathrm{~g} \text { of } \mathrm{Sm}+0.30 \mathrm{~g} \text { of Sm } \\
& =0.59 \mathrm{~g} \text { of } \mathrm{Sm}
\end{aligned}
$$

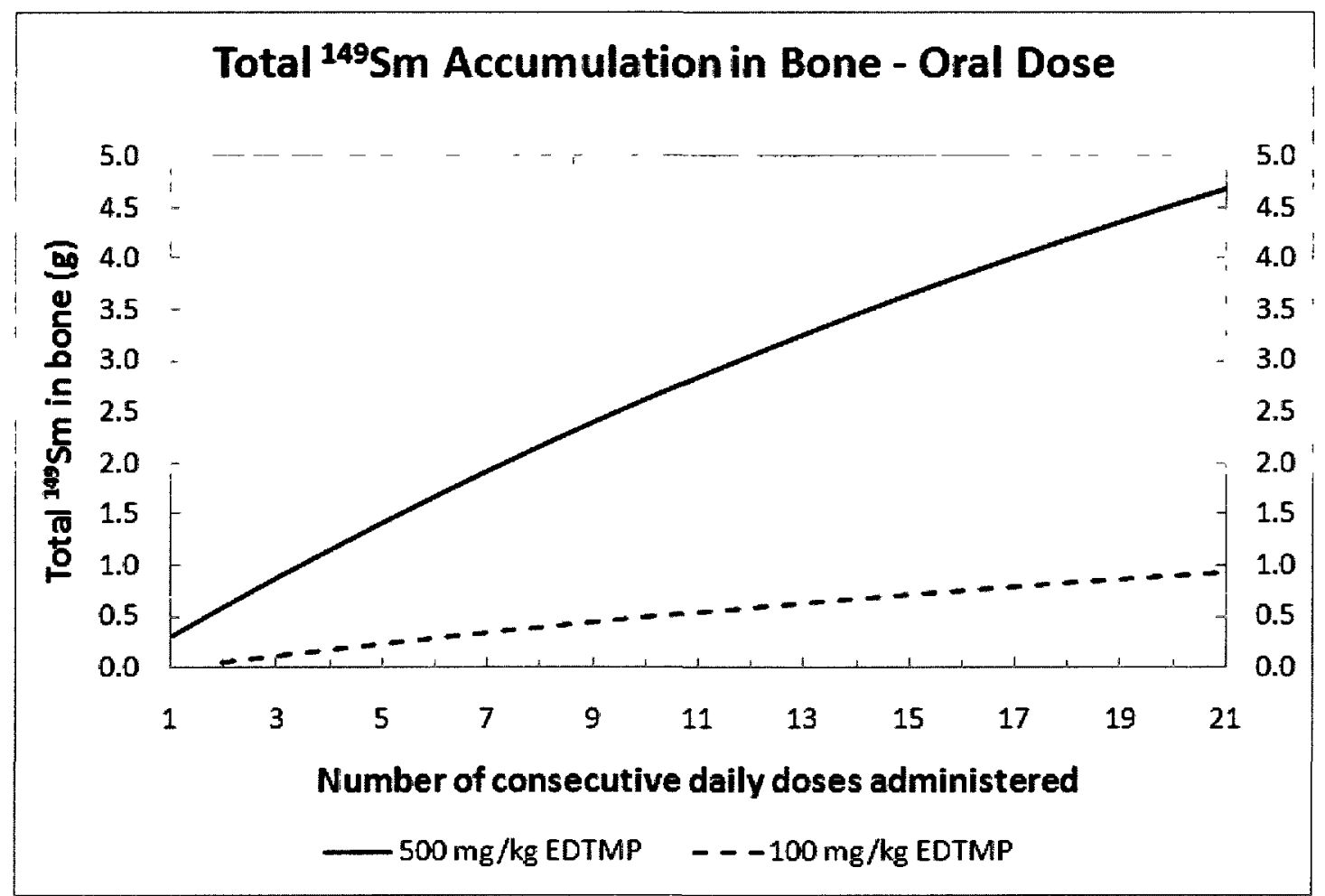

A plot of the total ${ }^{149} \mathrm{Sm}$ mass deposited on the skeleton from a daily oral dosing regimen of ${ }^{149} \mathrm{Sm}$-EDTMP. This takes into account the bioavailability of EDTMP as well as its natural rate of removal from bone (biological half-life). The patient is assumed to have an average mass of $70 \mathrm{~kg}$. 
APPENDIX B - TECHNICAL DRAWINGS AND PHOTOS OF THE 131I PHANTOM

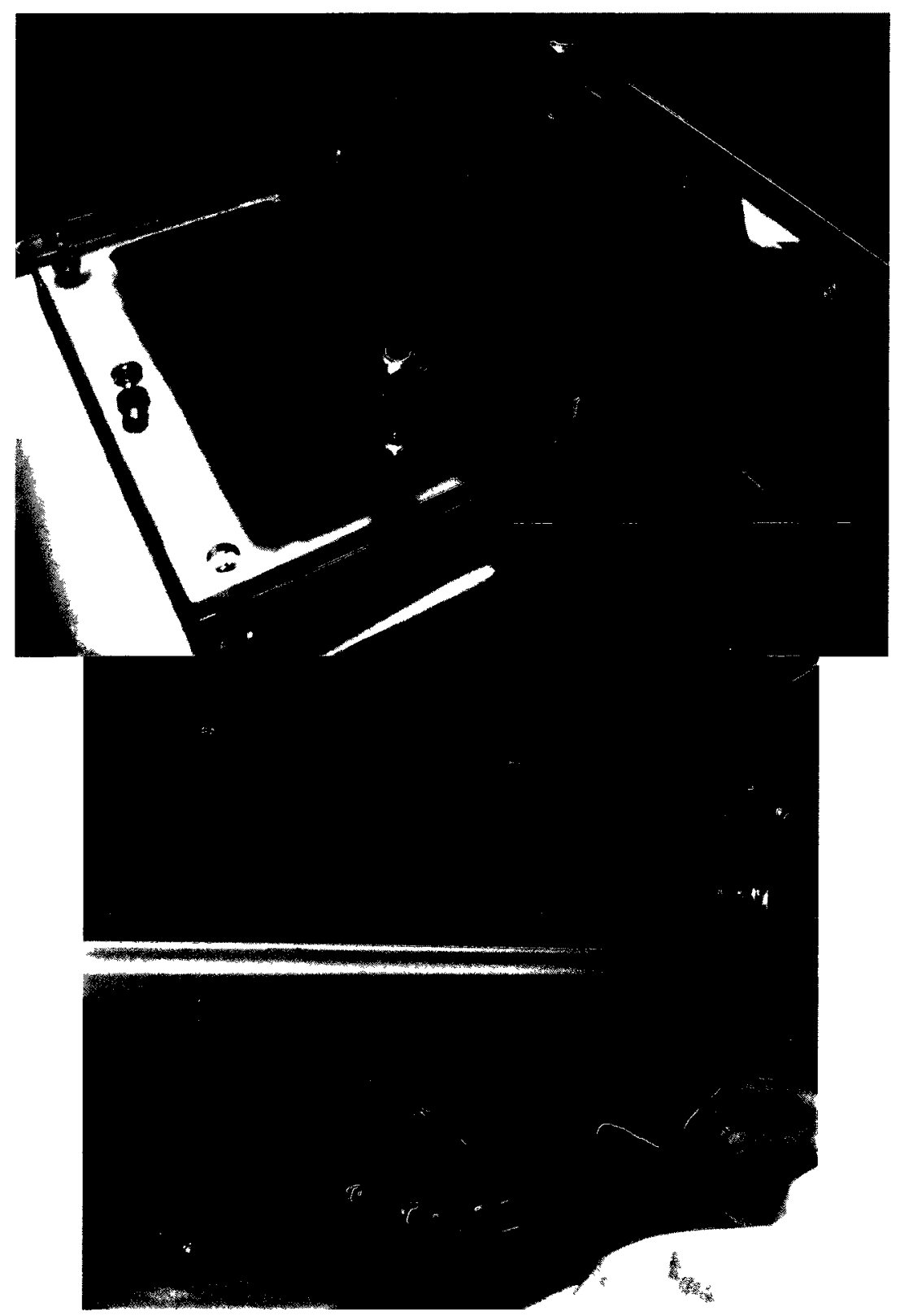

The Fill/Drain valves were threaded into the phantom lid. The lid was secured with an array of 8 bolts and a silicone gasket provided a watertight seal. The surface tension of water created problems with air bubbles forming in the $0.5 \mathrm{~cm}$ diameter lesions during filling. This was remedied by filling and draining the phantom using a soap solution prior to filling it with the ${ }^{131} \mathrm{I}$ solution. The soap residue was sufficient to allow filling without any trapped air. 


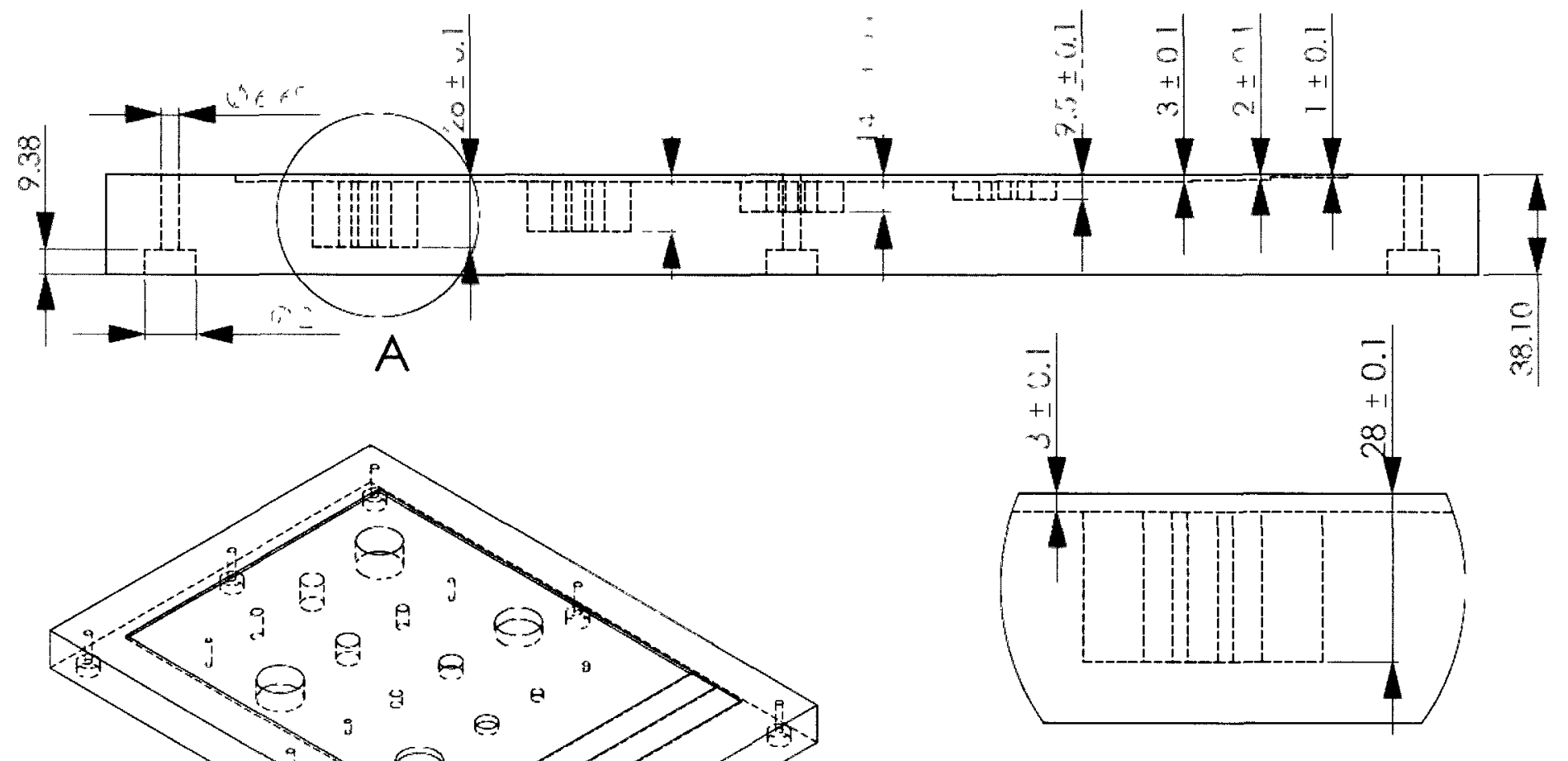

$$
\begin{aligned}
& \text { DETAIL A } \\
& \text { SCALE 1 : } 1 \\
& \text { IIILE: } \\
& \text { Phantom Base side view }
\end{aligned}
$$

UN-ESA ORERR WISE J

DIMENSIONS ARE

No special surface finish required. Transparency

TOLERANCES:

ミNG $\triangle P E R$

$+1-0.5 \mathrm{~mm}$ :FG AFPQ

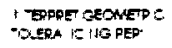

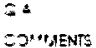

Acrylic

की 15-

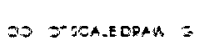

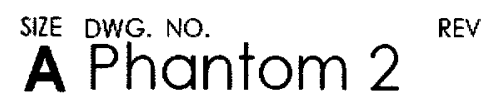

ACALE: 1:10 WEGH: 


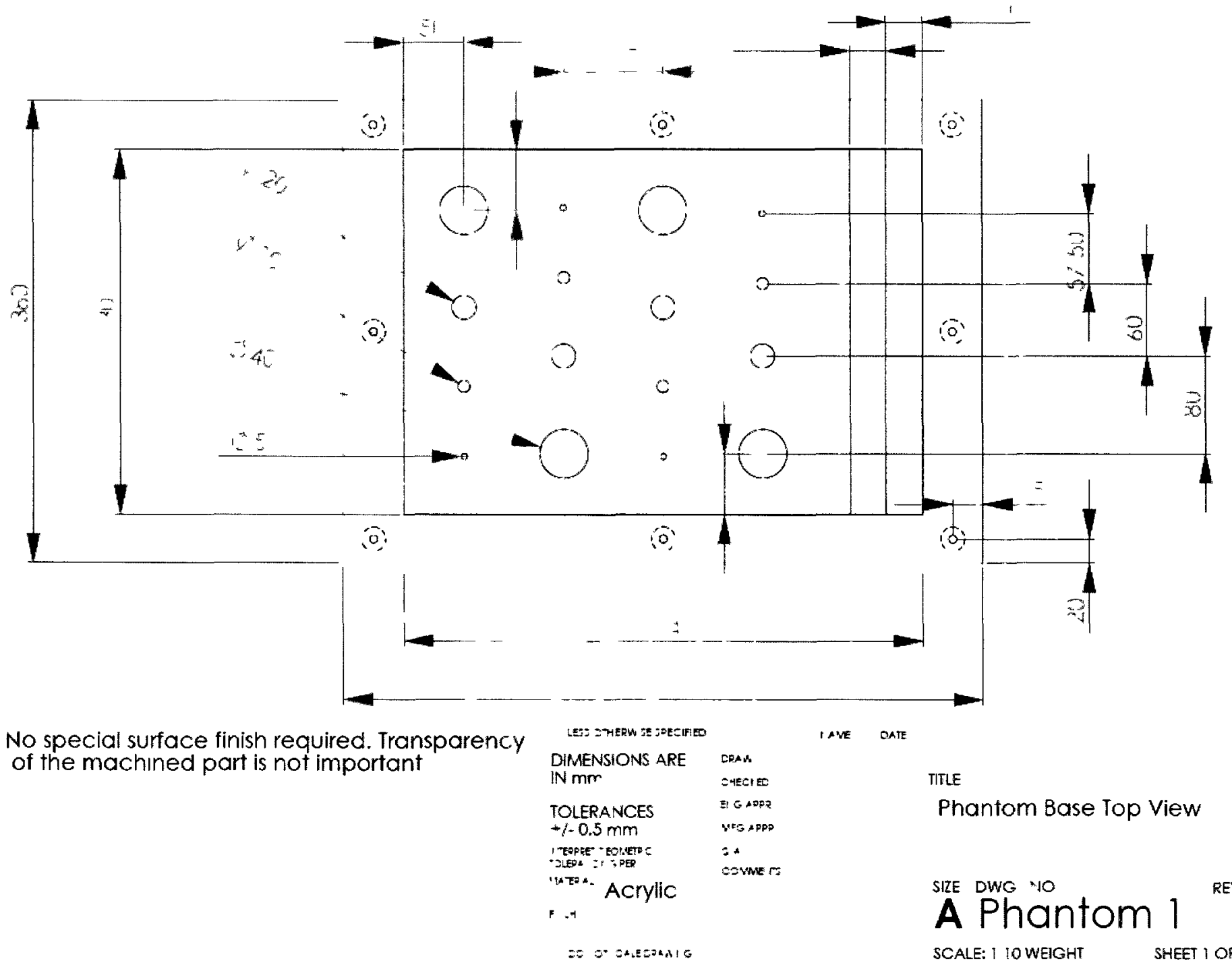

REV A Phantom I 

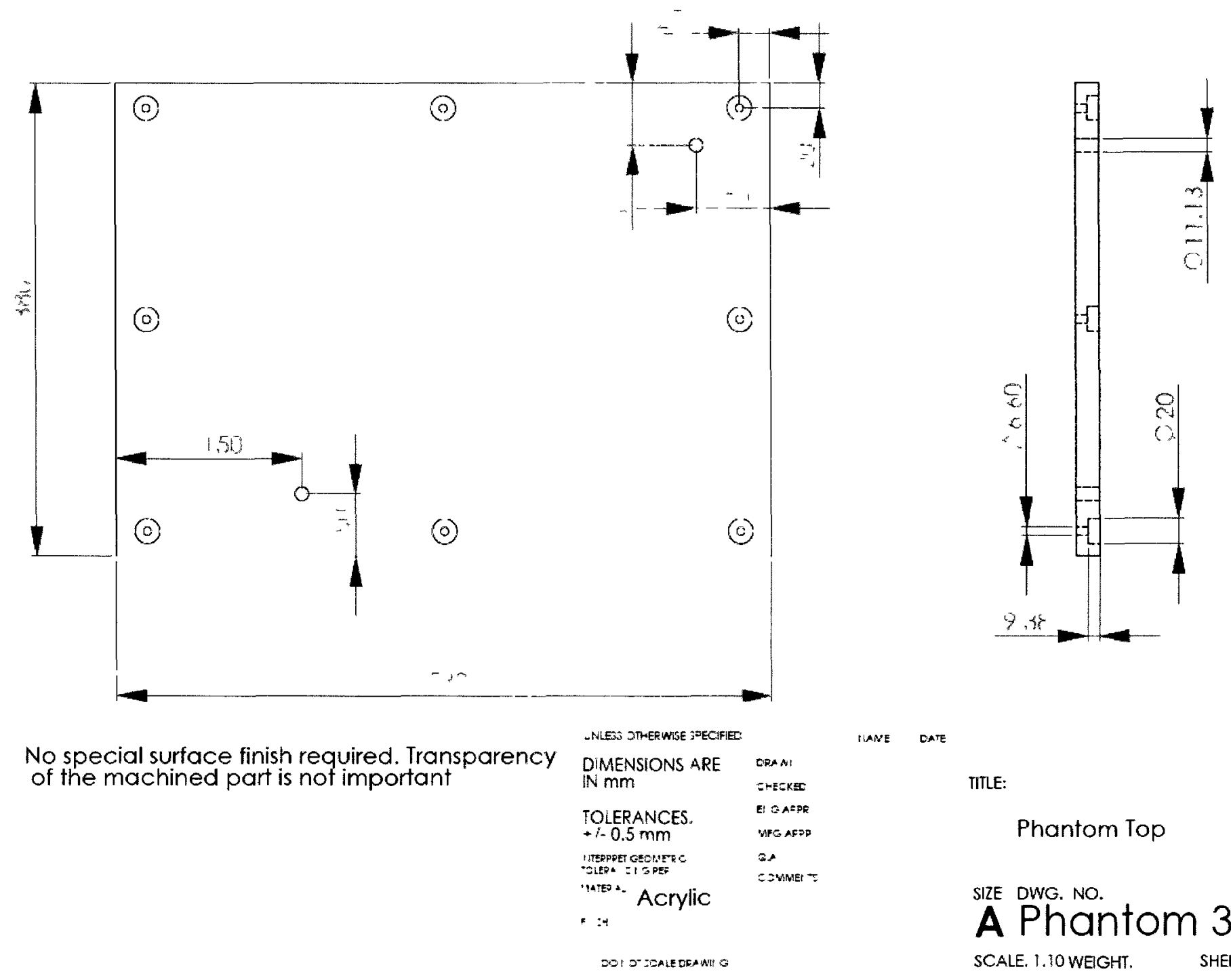

A Plze DWG. No.

SCALE. T. 10 WEGHT, SHEET I OF I 


\section{APPENDIX C - PHANTOM HOLE DEPTH ATTENUATION CORRECTION}

\section{CALCULATION}

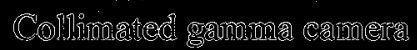

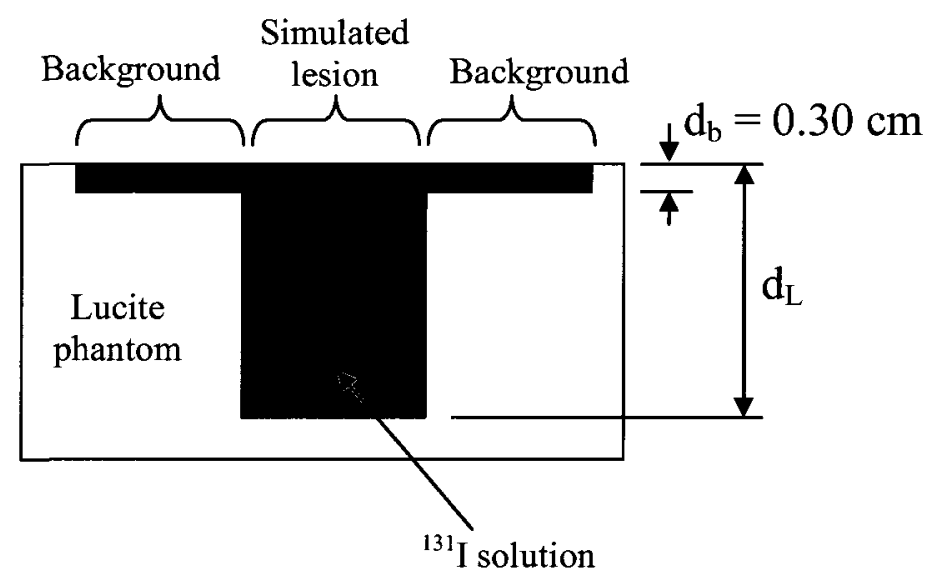

$\frac{A}{v}={ }^{131}$ I solution Activity per unit volume

$\mathrm{d}_{\mathbf{b}}=$ thickness of background cavity, representing healthy bone tracer uptake

$\mathrm{d}_{\mathrm{L}}=$ thickness of lesion cavity

$\mu_{\mathrm{H} 2 \mathrm{O}} @ 364 \mathrm{keV}=0.1106 \mathrm{~cm}^{-1} \quad$ ref: $(26)$

The phantom is designed to produce contrast between the simulated lesion and the background. The contrast is in the form of Activity per unit area from the gamma camera perspective. The deeper lesion cavity contains more ${ }^{131}$ I solution, which means that it will have a higher activity per unit area compared to the background region. However, some of the ${ }^{131}$ I gamma rays will be absorbed and/or scattered within the solution, so Activity per unit area does not scale linearly with cavity depth (for example, doubling the cavity depth will less than double the Activity per unit area observed by the gamma camera). Therefore, the cavity depth must be made deeper to correct for the self-attenuation of the solution. 
The equation for fractional transmission due to self-absorption is (13):

$$
t_{S}=\frac{\text { observed activity per unit area }}{\text { total activity per unit area }}=\frac{1-e^{-\mu d}}{\mu d} \leq 1
$$

where $d$ is the depth of the Active solution and $\mu$ is the linear attenuation coefficient of the solution. Because the gamma camera is collimated, we are only concerned about attenuation in the direction perpendicular to the camera.

If the total activity per unit area (parallel to the gamma camera plane) in the lesion is:

$$
\text { total activity per unit area }=\frac{A}{v} \times d_{L}
$$

Then we can define the observed activity per unit area as:

$$
\text { observed activity per unit area }=\frac{A}{v} \times d_{L, \text { effective }}
$$

In the case of 8:1 lesion-to-background contrast (which is the 15:1 tracer uptake ratio, see Section 3.4.2), we desire:

$$
d_{L, e f f e c t i v e}=8 \times d_{b, e f f e c t i v e}
$$

Verifying the fractional transmission for the background region:

$$
t_{S, b}=\frac{1-e^{-\mu d_{b}}}{\mu d_{b}}=\frac{1-e^{-\left(0.1106 \mathrm{~cm}^{-1}\right)(0.30 \mathrm{~cm})}}{\left(0.1106 \mathrm{~cm}^{-1}\right)(0.30 \mathrm{~cm})}=0.98
$$

There is very little self-attenuation in the $0.30 \mathrm{~cm}$ of solution in the background region of the phantom. Therefore, we can assume that $d_{b} \approx d_{b, \text { effective }}$ and:

$$
d_{L, \text { effective }}=8 \times d_{b, \text { effective }}=8 \times 0.30 \mathrm{~cm}=2.4 \mathrm{~cm}
$$


Combining equations 16,17 , and 18 and evaluating the simulated lesion:

$$
\begin{aligned}
\frac{\frac{A}{\vartheta} \times d_{L, e f f e c t i v e}}{\frac{A}{\eta}} \times d_{t} & =\frac{1-e^{-\mu d_{L}}}{\mu d_{t}} \\
d_{L, \text { effective }} & =\frac{1-e^{-\mu d_{L}}}{\mu}
\end{aligned}
$$

Rearranging equation 20 to solve for $d_{L}$ :

$d_{L}=\frac{-\ln \left(1-\mu d_{L, \text { effective }}\right)}{\mu}=\frac{-\ln \left[1-\left(0.1106 \mathrm{~cm}^{-1}\right)(2.4 \mathrm{~cm})\right]}{0.1106 \mathrm{~cm}^{-1}}=2.8 \mathrm{~cm}$

Therefore, in order for the observed lesion-to-background activity per unit area ratio to be $8: 1$, the ratio of $d_{L}: d_{b}$ must be approximately $9.3: 1$ 


\section{APPENDIX D - ETNA CONFIGURATION}

The following images demonstrate the parameters used in the ETNA efficiency transfer calculations performed for this research.

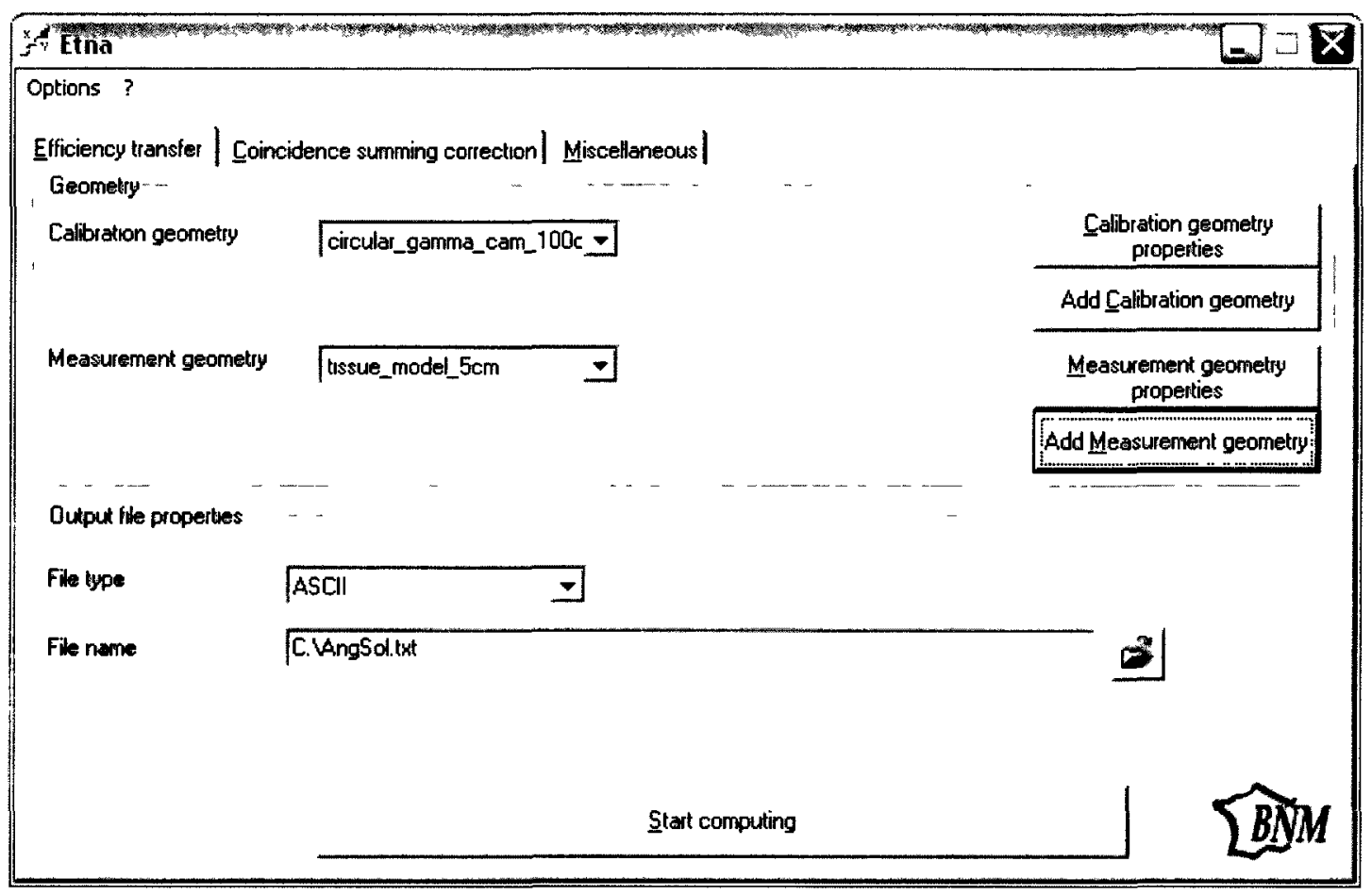

ETNA home window. The Calibration geometry (reference model and data) and Measurement geometry (new efficiency model) are selected here. Each model can be edited by clicking on the appropriate button. The computation is also initiated from here. 


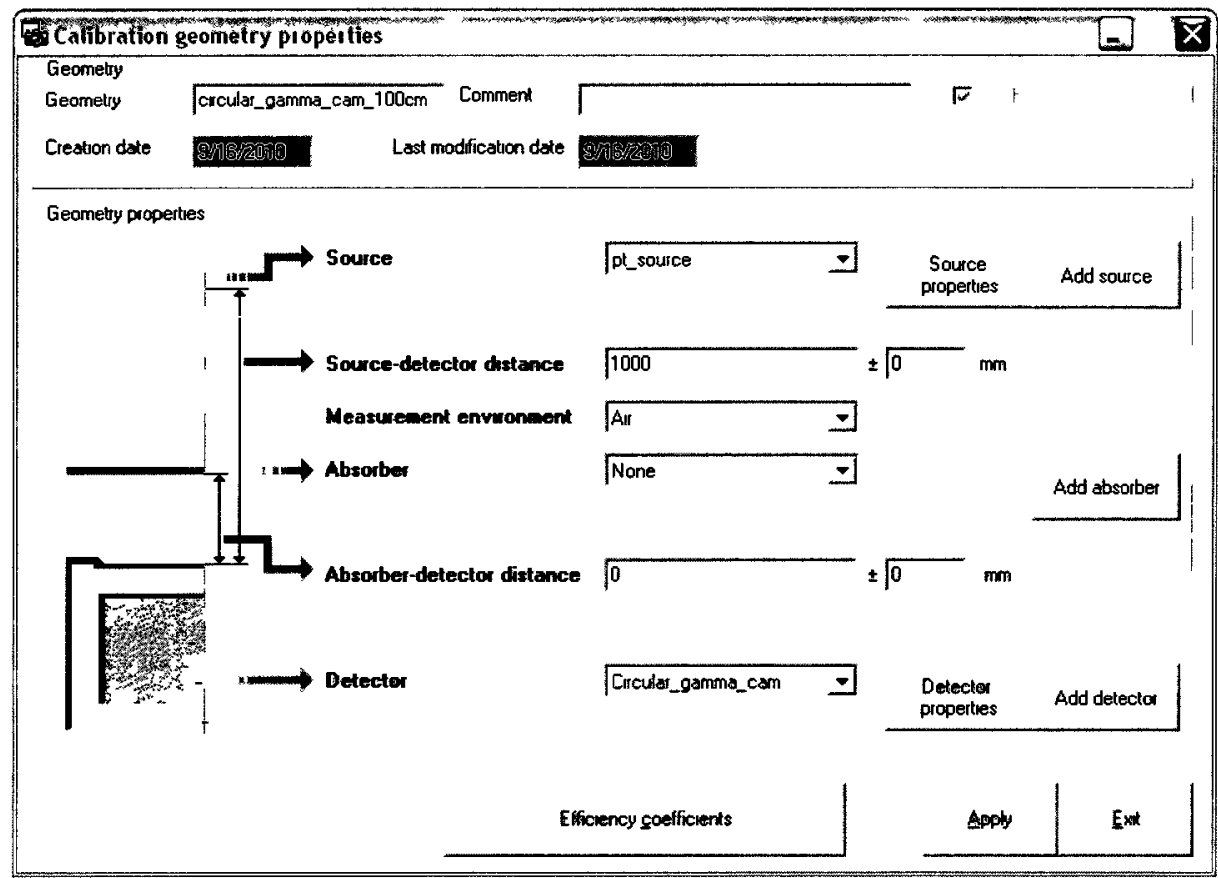

The Calibration Geometry properties window. The physical parameters of the calibration geometry are defined here. Source, detector, and absorber models and calibration efficiency coefficients are selected/added here.

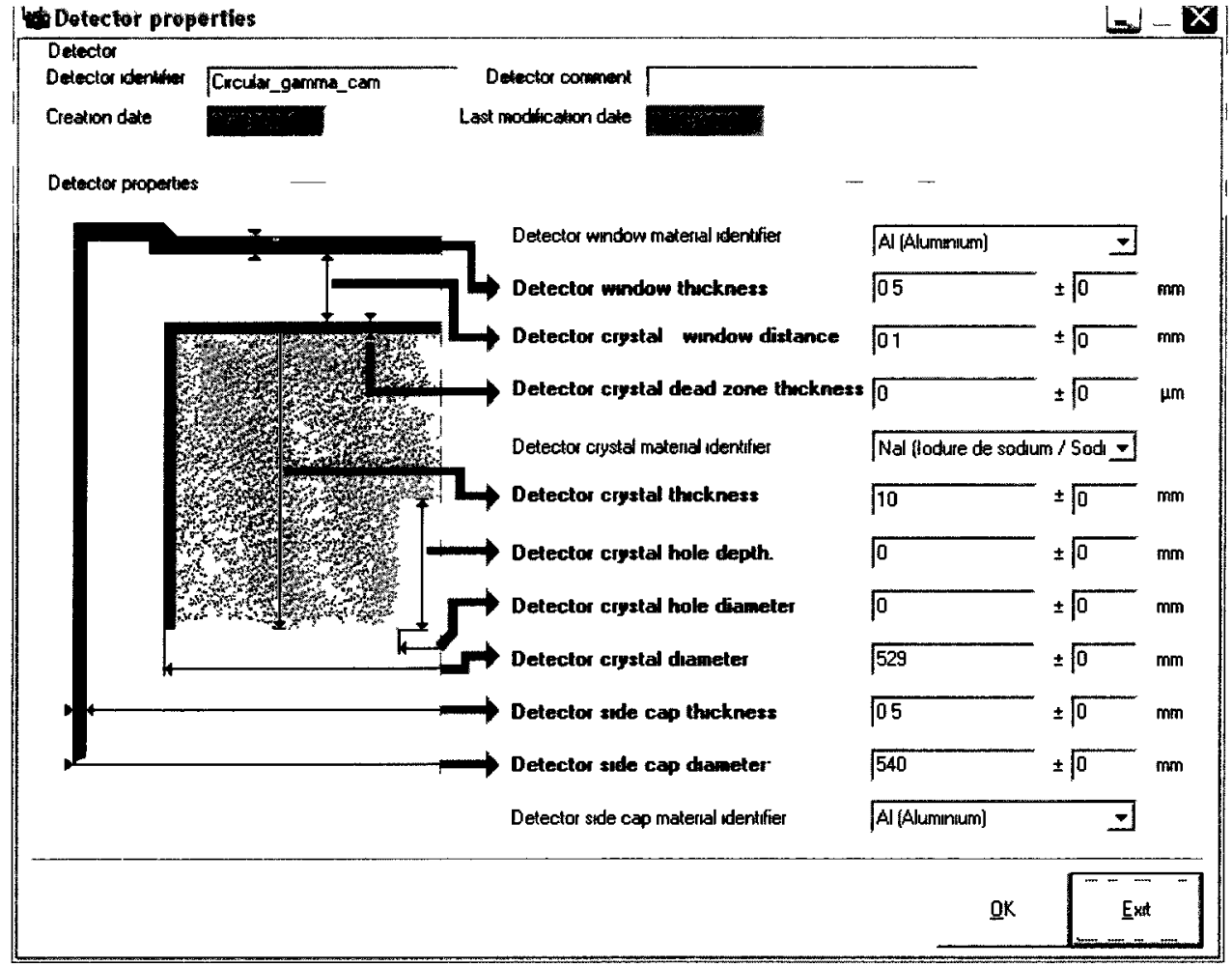

The Detector Properties window The physical parameters of the detector are defined here 


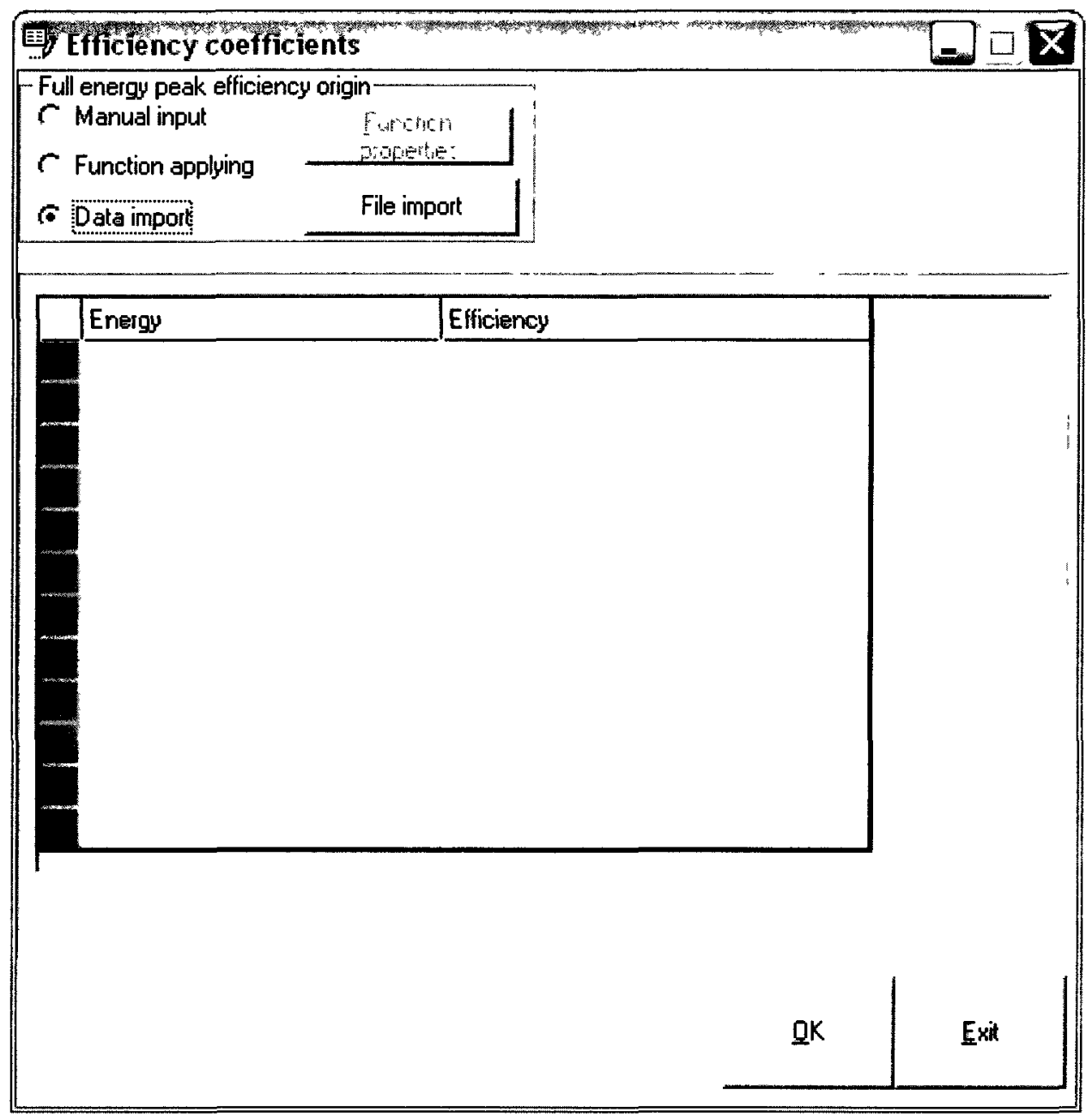

The Calibration Efficiency Coefficients window. These are the FEPE values from literature for the Gamma Camera, which are used to calculate the new efficiency coefficients corresponding to the Measurement geometry. 


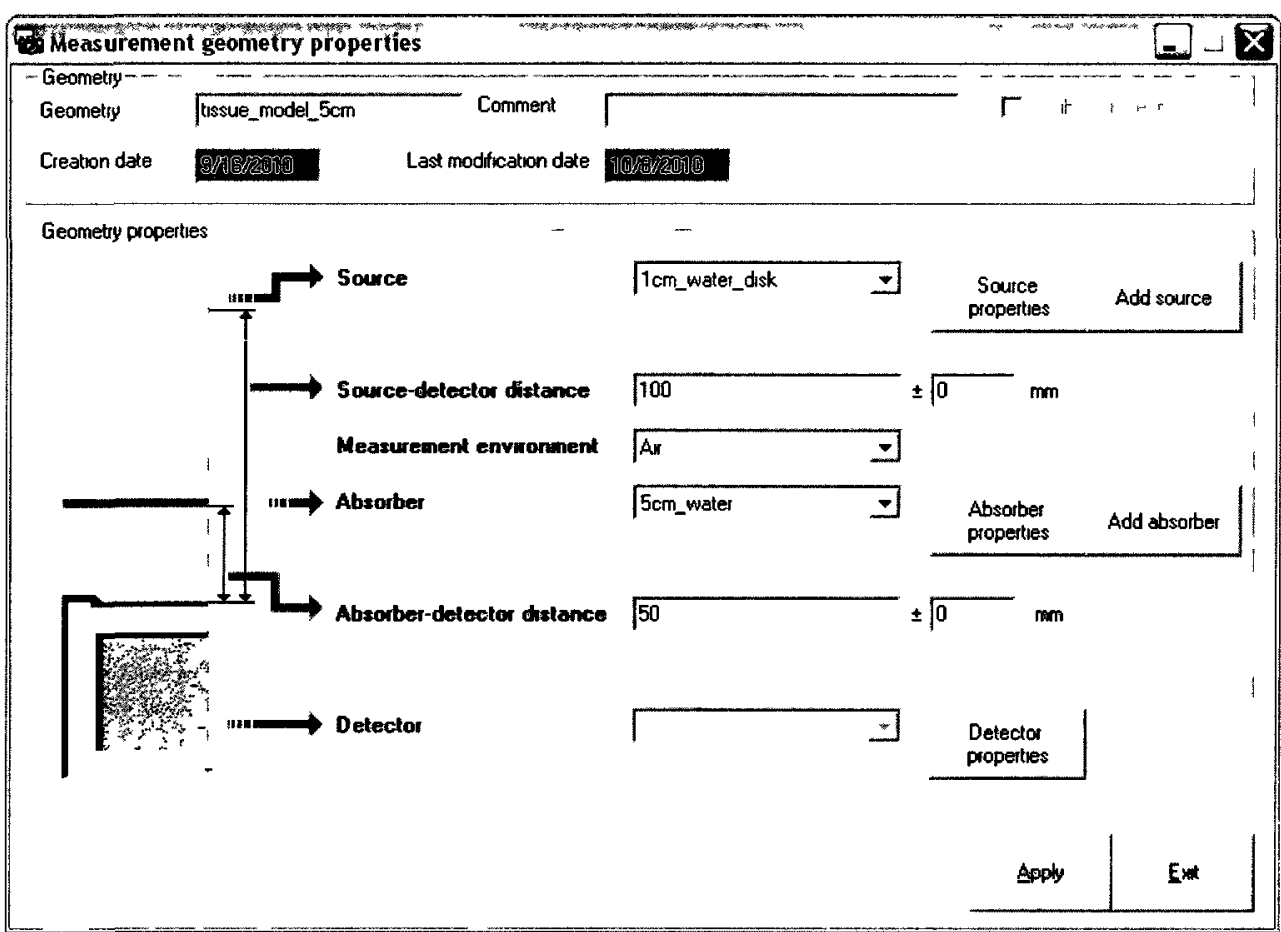

The Measurement Geometry properties window. Identical to the Calibration Geometry properties window, except that the detector model cannot be modified and there are no efficiency coefficients to upload, since they are the output of the ETNA calculations. The Absorber is a different thickness and the Source-Detector Distance changes for each of the 12 tissue model layers (see table below).

Depth position $0 \mathrm{~cm}$ corresponds to the point closest to the neutron source (i.e., furthest from the detector).

\begin{tabular}{|ccc|}
\hline Depth position (cm) in tissue model & Source-Detector Distance & Absorber Thickness \\
\hline \hline $0-1$ & 160 & $11 \mathrm{~cm}$ \\
$1-2$ & 150 & $10 \mathrm{~cm}$ \\
$2-3$ & 140 & $9 \mathrm{~cm}$ \\
$3-4$ & 130 & $8 \mathrm{~cm}$ \\
$4-5$ & 120 & $7 \mathrm{~cm}$ \\
$5-6$ & 110 & $6 \mathrm{~cm}$ \\
$6-7$ & 100 & $5 \mathrm{~cm}$ \\
$7-8$ & 90 & $4 \mathrm{~cm}$ \\
$8-9$ & 80 & $3 \mathrm{~cm}$ \\
$9-10$ & 70 & $2 \mathrm{~cm}$ \\
$10-11$ & 60 & $1 \mathrm{~cm}$ \\
$11-12$ & 50 & $0 \mathrm{~cm}$ \\
\hline
\end{tabular}




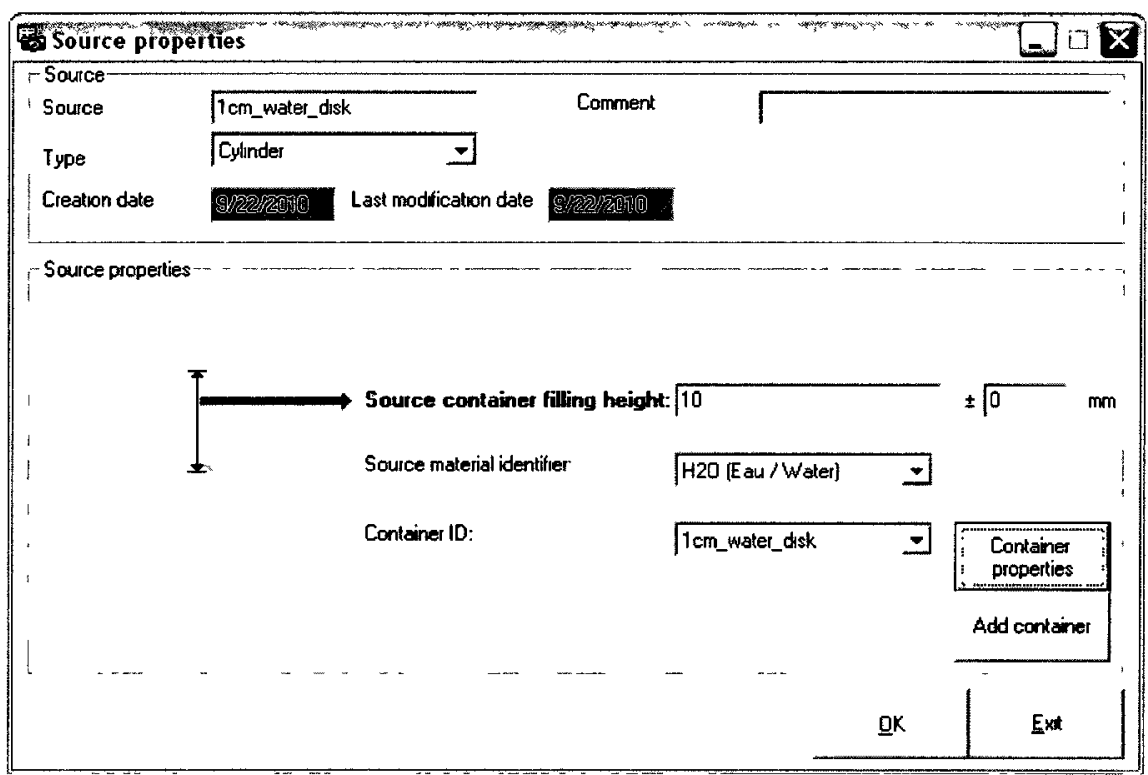

The Measurement geometry source properties window. The $1 \mathrm{~cm}$ thick discs of the tissue model are defined here.

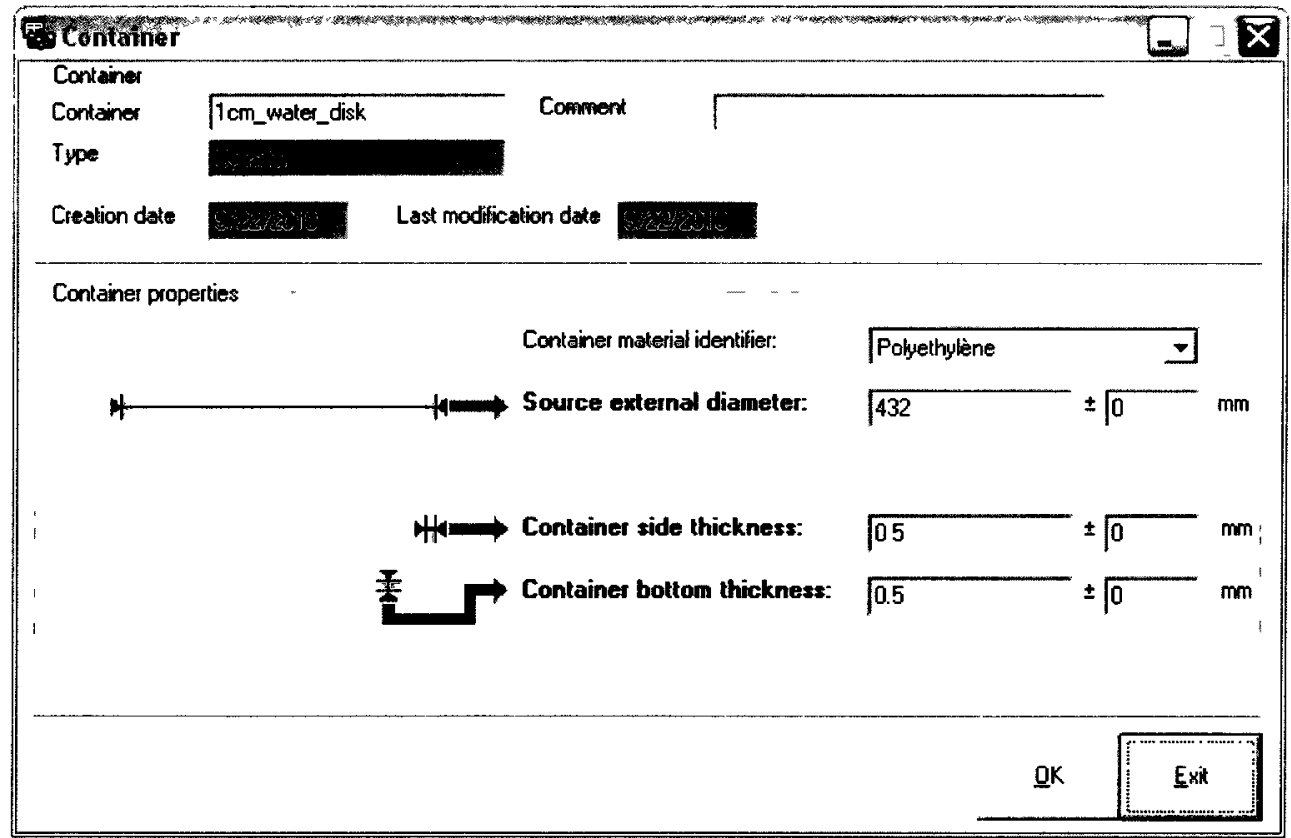

Additional Measurement geometry source properties are defined here. 
APPENDIX E - SIGNAL ATTENUATION AND CORRECTION FACTOR FOR THE THIGH TISSUE MODEL

\begin{tabular}{|rrrr|}
\hline Table of Constants & & & \\
\hline Linear attenuation coefficient of water, $\mu_{\mathrm{H} 2 \mathrm{O}}, @ 364 \mathrm{keV}=$ & 0.111 & $\mathrm{~cm}^{-1}$ & Ref: (26) \\
Linear attenuation coefficient of Lucite, $\mu_{\text {Lucite, }} @ 364 \mathrm{keV}=$ & 0.127 & $\mathrm{~cm}^{-1}$ & Ref: (26) \\
Lucite phantom lid thickness $=$ & $1.9 \mathrm{~cm}$ & \\
Thigh tissue thickness $=$ & $6.0 \mathrm{~cm}$ & \\
\hline
\end{tabular}

$$
\begin{aligned}
\text { Actual }{ }^{131} \text { I transmission through Lucite } & =e^{-\left(\mu_{\text {Lucite }}\right)\left(x_{\text {Lucite }}\right)} \\
& =e^{-\left(0.127 \mathrm{~cm}^{-1}\right)(1.9 \mathrm{~cm})} \\
& =79 \%
\end{aligned}
$$

Theoretical ${ }^{131}$ I transmission through thigh tissue $=e^{-\left(\mu_{H 2 O}\right)\left(x_{t i s s u e}\right)}$

$$
\begin{aligned}
& =e^{-\left(0.111 \mathrm{~cm}^{-1}\right)(6.0 \mathrm{~cm})} \\
& =51 \%
\end{aligned}
$$

Ratio of actual - to - theoretical transmission $=\frac{0.79}{0.51}=1.5$

There is more attenuation (i.e., less transmission) though the thigh tissue model compared to the ${ }^{131}$ I phantom. Therefore, the count rate through the thigh tissue model is lower, so it would take 1.5 times longer to generate an image through $6 \mathrm{~cm}$ of tissue compared to $1.9 \mathrm{~cm}$ of Lucite. 


\section{References}

1. hopitalmanagement.net. Short of Answers: The Ongoing Isotope Shortage. hopitalmanagement.net. [Online] Net Resources International, March 15, 2010. [Cited: November 21, 2010.] http://www.hospitalmanagement.net/features/feature79330/.

2. Society of Nuclear Medicine. Fast Facts on the Worldwide Medical Isotope Crisis. SNM: Advancing Molecular Imaging and Therapy. [Online] Society of Nuclear Medicine (SNM), June 15, 2009. [Cited: November 21, 2010.] http://interactive.snm.org/index.cfm?PageID=8803.

3. Goodhand, Peter; Drouin, Richard; Mason, Thom; Turcotte, Eric; Report of the Expert Review Panel on Medical Isotope Production. Ottawa, 2009.

4. Shaw, Gina. Medical Isotope Shortage Nearing End-For Now. Clinical Oncology News. [Online] McMahon Publishing, October 2010. [Cited: November 21, 2010.]

http://www.clinicaloncology.com/index.asp?section_id=150\&show=dept\&issue_id=674 \&article_id=16057.

5. Gould, Paula. Medical isotope shortage reaches crisis level. Nature News. [Online] Nature Publishing Group, a division of Macmillan Publishers, July 15, 2009. [Cited: November 21, 2010.] http://www.nature.com/news/2009/090715/full/460312a.html.

6. Ballinger, J. R. Short- and long-term responses to molybdenum-99 shortages in nuclear medicine. The British Journal of Radiology. November 2010. Vol. 83, pp. 899901.

7. Bronstein, David and Jakubiak, David. Nuclear Pharmacists Fret Over Tech99m Shortage. Pharmacy Practice News. [Online] McMahon Publishing, December 2009. [Cited: November 21, 2010.]

http://www.pharmacypracticenews.com/index.asp?show=dept\&section_id=439\&issue_id $=587 \&$ article_id=14356.

8. The Globe and Mail. Precious medical isotopes could be shipped overseas . The Globe and Mail. [Online] CTVglobemedia Publishing Inc., March 7, 2010. [Cited: November 22, 2010.] http://www.theglobeandmail.com/news/national/precious-medicalisotopes-could-be-shipped-overseas/article1492961/. 
9. National Resources Canada. GOVERNMENT OF CANADA ACTIONS TO ADDRESS THE MEDICAL ISOTOPE SHORTAGE. National Resources Canada, The Media Room. [Online] Government of Canada, 2009. [Cited: Novermber 21, 2010.] http://www.nrcan-rncan.gc.ca/media/newcom/2009/200967a-eng.php.

10. Natural Resources Canada. Government of Canada Investing in Isotope Innovation. Natural Resources Canada. [Online] January 24, 2011. [Cited: March 30, 2011.] http://www.nrcan.gc.ca/media/newcom/2011/201113eng.php?PHPSESSID=47337b0ea5b85dc87e5ff62a112b1 d8b.

11. Henkin, Robert E. Nuclear Medicine. 2nd Edition. Philadelphia : Mosby Elsevier, 2006.

12. Adelphi Technology, Inc. Neutron Generator Products. Adelphi Technology, Inc. [Online] Adelphi Technology, Inc., 2009. [Cited: November 21, 2010.] http://www.adelphitech.com/products.html.

13. Molnar, Gabor L. Handbook of Prompt Gamma Activation Analysis. Dordrecht, The Netherlands : Kluwer Academic Publishers, 2004.

14. Prince, Jerry L. and Links, Jonathan M. Medical Imaging Signals and Systems. Upper Saddle River : Pearson Prentice Hall, 2006. ISBN 0-13-065353-5.

15. Radiation dose to technicians per nuclear medicine procedure: comparison between technetium- $99 \mathrm{~m}$, gallium-67, and iodine-131 radiotracers and fluorine-18 fluorodeoxyglucose. Chiesa, C.; De Sanctis, V.; Crippa, F.; Schiavini, M.; Fraigola, C.E.; Bogni, A.; Pascali, C.; Decise, D.; Marchesini, R.; Bombardieri, E.; 1997, European Journal of Nuclear Medicine, Vol. 24, pp. 1380-1389.

16. Karesh, Stephen M. Principles of Radiopharmacy. [book auth.] Robert E. Henkin. Nuclear Medicine, 2nd edition. Philadelphia : Mosby Elsevier, 2006, Vol. I.

17. Augustine, S. C.; Rutar, F. J.; Holdeman, K. P.; Jacobson, D. A.; Dalrymple, G. V.; Radionuclide Generators: Design, Operation, and Clinical Utility. [book auth.] R. E. Henkin. Nuclear Medicine. 2nd Edition. Philadelphia : Mosby Elsevier, 2006, Vol. I.

18. Wernick, Miles N. and Aarsvold, John N. Emission Tomography: The Fundamentals of PET and SPECT. San Diego : Elsevier Academic Press, 2004. ISBN: 012-744482-3. 
19. Bombardieri, Emilio; Aktolun, Cumali; Baum, Richard P.; BishofDelaloye, Angelica; Buscombe, John; Chatal, Jean Francois; Maffioli, Lorenzo; Moncayo, Roy; Mortelmans, Luc; Reske, Sven N.; BONE SCINTIGRAPHY PROCEDURES GUIDELINES FOR TUMOUR IMAGING. Oncology Committee of the European Association of Nuclear Medicine, September 2, 2004.

20. Donohoe, Kevin J.; Brown, Manuel L.; Collier, B. David; Henkin, Robert E.; Carretta, Robert F.; O'Mara, Robert E.; Royal, Henry D.; Society of Nuclear Medicine Procedure Guideline for Bone Scintigraphy version 3.0. Reston, Virginia, United States of America : Society of Nuclear Medicine, June 20, 2003.

21. Goeckeler, W. F.; Edwards, B.; Volkert, W. A.; Holmes, R. A.; Simon, J.; Wilson, D.; Skeletal Localization of Samarium-153 Chelates: Potential Therapeutic Bone Agents. Journal of Nuclear Medicine. Freeport, Texas, USA, April 1987. Vol. 28, pp. 28:495-504.

22. Bayouth, John E.; Macey, Daniel J.; Kasi, Leela P.; Fossela, Frank V; Dosimetry and Toxicity of Samarium-153-EDTMP Administered for Bone Pain Due to Skeletal Metastases. Journal of Nuclear Medicine. Houston, Texas, 1994. Vol. 35, pp. 63-69.

23. Deng, Houfu. Radiopharmaceutical (Sm-153-EDTMP) therapy of skeletal metastases: clinical application in 350 patients. The Journal of Radiology. Chengdu, China, August 2002.

24. Gunter, Donald L. and Halama, James R. Gamma Camera Collimator Characteristics and Design. [book auth.] Robert E. Henkin. Nuclear Medicine. 2nd Edition. Philadelphia, Pennsylvania : Mosby Elsevier, 2006.

25. Siemens Medical Solutions USA, Inc. Symbia TruePoint SPECT-CT System Specifications. Hoffman Estates, Illinois, USA, 2005.

26. Johns, H. E. and Cunningham, J. R. The Physics of Radiology, 4th ed. Springfield, Illinois : Charles C Thomas, 1983. 0-398-04669-7.

27. Madsen, Mark T. Scintillation Detectors and Scintillation Detector Counting Systems. [book auth.] Robert E. Henkin. Nuclear Medicine. 2nd Edition. Philadelphia, Pennsylvania : Mosby Elsevier, 2006.

28. Halama, James and Simmons, Guy. Gamma Camera Imaging Systems. [book auth.] Robert E. Henkin. Nuclear Medicine. 2nd Edition. Philadelphia : Mosby Elsevier, 2006. 
29. Procedure Guideline for SPECT/CT Imaging 1.0. Delbeke, D.; Coleman, R.; Guiberteau, M.; Brown, M.; Royal, H.; Siegel, B.; Townsend, D.; Berland, L.; Parker, A.; Zubal, G; Cronin, V.; Society of Nuclear Medicine, Inc., 2006. http://www.snm.org/guidelines.

30. Martin, C J, Sharp, P F and Sutton, D G. Measurement of image quality in diagnostic radiology. Applied Radiation and Isotopes. Pergamon, 1999. Vol. 50, pp. $21-$ 38.

31. Burgess, A E. The Rose Model, revisited. Journal of the Optical Society of America. Boston, 1999. Vol. 16.

32. Diaz, M P; Rizo, O D; Diaz, A L; Aparicio, E E; Diaz, R R; Activity optimization method in SPECT: A comparison with ROC analysis. Journal of Zhejiang University. 2006. Vol. 7.

33. Glick, Stephen. Image Content and Image Filtering Techniques. [book auth.] Robert E. Henkin. Nuclear Medicine. 2nd Edition. Philadelphia, Pennsylvania : Mosby, Elsevier, 2006.

34. Spanoudaki, V. C. and Ziegler, S. I. PET \& SPECT Instrumentation. [book auth.] W. Semmler and M. Schwaiger. Molecular Imaging I. Handbook of Experimental Pharmacology. Springer-Verlag Berlin Heidelberg, 2008.

35. Halama, J. R. Representation of Gamma Camera Images by Computer. [book auth.] R. E. Henkin. Nuclear Medicine. 2nd Edition. Philadelphia : Mosby Elsevier, 2006, Vol. 1.

36. United States Nuclear Regulatory Commission. Units of Radiation Dose. United States Nuclear Regulatory Commission. [Online] July 09, 2009. [Cited: July 22, 2009.] http://www.nrc.gov/reading-rm/doc-collections/cfr/part020/part020-1004.html.

37. Stabin, Michael G. Internal Radiation Dosimetry. [book auth.] Robert E. Henkin. Nuclear Medicine. 2nd Edition. Philadelphia : Mosby Elsevier, 2006.

38. Radiation Doses to Staff in a Nuclear Medicine Department. Ho, W Y; Wong, K K; Leung, Y L; Cheng, K C; Ho, F T.H.; 2002, J HK Coll Radiol, pp. 5:2428 .

39. Berger, M. J.; Hubbell, J. H.; Seltzer, S. M.; Chang, J.; Coursey, J. S.; Sukumar, R.; Zucker, D. S.; Olsen, K.; XCOM: Photon Cross Sections Database. [Online] National Institute of Standards and Technology (NIST), 1998. http://www.nist.gov/pml/data/xcom/index.cfm. 
40. Korean Atomic Energy Research Institute. Table of Nuclides. Nuclear Data Evaluation Lab. [Online] 2000. [Cited: July 15, 2009.] http://atom.kaeri.re.kr/.

41. Sauerwein, W. and Zurlo, A. The EORTC Boron Neutron Capture Therapy (BNCT) Group: achievements and future projects. European Journal of Cancer. Pergamon, 2002. Vol. 38.

42. Binns, P. J., Riley, K. J. and Harling, O. K. Epithermal Neutron Beams for Clinical Studies of Boron Neutron Capture Therapy: A Dosimetric Comparison of Seven Beams. Radiation Research. Radiation Research Society, 2005. Vol. 164, pp. 212-220.

43. Atanackovic, J.; Grinyer, J.; Chettle, D. R.; Byun, S. H.; The comparison of two MCNP models used for prompt gamma in vivo detection of cadmium and mercury. Nuclear Instruments and Methods in Physics Research B. Hamilton, ON, Canada : Elsevier, 2007. Vol. 263.

44. Grinyer, J., Byun, S. H. and Chettle, D. R. In vivo prompt gamma neutron activation analysis of cadmium in the kidney and liver. Applied Radiation and Isotopes. Elsevier, 2005. Vol. 63.

45. Scientific Committee on Health and Environmental Risks (SCHER). Non surfactant Organic Ingredients and Zeolite-based Detergents. European Commission, Health \& Consumer Protection DG, 2007.

46. Calvin, G.; Long, P. H.; Stitzel, K. A.; Anderson, R. L.; Balmbra, R. R.; Bruce, R. D.; Ethylenediaminetetra (Methylenephosphonic acid): Genotoxicity, Biodistribution, and subchronic and chronic toxicity in Rats. Food and Chemical Toxicity, Great Britain : Pergamon Press, 1988. Vol. 26, pp. 601-610.

47. International Atomic Energy Agency (IAEA). Optimization of Production and Quality Control of Therapeutic Radionuclides and Radiopharmaceuticals. Vienna, Austria : Industrial Applications and Chemistry Section - IAEA, 1999.

48. Sartor, Oliver; Reid, Robert H.; Bushnell, David L.; Quick, Donald P.; Ell, Peter J.; Safety and Efficacy of Repeat Administration of Samarium Sm-153 Lexidronam to Patients With Metastatic Bone Pain. Cancer. 2007. Vol. 109.

49. Bone, Henry G. and Schurr, Werner. Intravenous Bisphosphonate Therapy for Osteoporosis: Where Do We Stand? Current Osteoporosis Reports. 2004. Vol. 2. ISSN 1544-1873.

50. Los Alamos National Laboratory. MCNP - A General Monte Carlo NParticle Transport Code - Version 5. MCNP. [Online] Los Alamos National Security (LANS), 2006. [Cited: April 12, 2011.] https://mcnpx.lanl.gov/. 
51. PerkinElemer Inc. Iodine-131 Handling Precautions. Safe Handling Guide: Radiochemicals. Waltham, MA, USA, 2007.

52. Fuller, N. J.; Hardingham, C. R.; Graves, M.; Screaton, N.; Dixon, A. K.; Ward, L. C.; Elia, M.; Predicting composition of leg sections with anthropometry and bioelectrical impedance analysis, using magnetic resonance imaging as reference.

Clinical Science, Great Britain : The Biochemical Society and the Medical Research Society, 1999. pp. 647-657.

53. Tortora, Gerard J. and Derrickson, Bryan. Principles of Anatomy and Physiology, John Wiley \& Sons, Inc., 2006. ISBN-13: 978-0-471-68934-3.

54. Engdahl, J. C. and Bharwani, K. Suitability of nuclear medicine gamma cameras as gamma spectrometers in the event of a radiological emergency. Nuclear Instruments and Methods in Physics Research A, Elsevier, 2005. pp. 569-577.

55. RESPONSE FUNCTIONS FOR SODIUM IODIDE SCINTILLATION DETECTORS. Berger, M. J. and Seltzer, S. M., NORTH-HOLLAND PUBLISHING CO., 1972, Vol. 104, pp. 317-332.

56. Ichihara, Takashi; Ogawa, Koichi; Motomura, Nobutoku; Kubo, Atushi; Hashimoto, Shozo; Compton Scatter Compensation Using the Triple-Energy Window Method for Single- and Dual-Isotope SPECT. Journal of Nuclear Medicine. 1993. Vol. 34, pp. 2216-2221.

57. Hannequin, P. and Mas, J. Statistical and heuristic image noise extraction (SHINE) -- a new method for processing Poisson noise in scintigraphic images. Physics in Medicine and Biology. 2002.

58. Yahil, A., Vija, A. H. and Hawman, E. G. Enhanced Feature Extraction in Planar Nuclear Medicine Using Pixon Minimum-Complexity Image Processing. Nuclear Science Symposium Conference Record. IEEE, 2006.

59. Design of an epithermal column for BNCT based on D-D fusion neutron facility. Durisi, E.; Zanini, A.; Manfredotti, C.; Palamara, F.; Sarotto, M.; Visca, L.; Nastasi, U.; Elsevier, 2007, Nuclear Instruments and Methods in Physics Research A, Vol. 574, pp. 363-369.

60. ANSTO Radiopharmaceuticals and Industrials. Product Information: Quadramet. ANSTO Radiopharmaceuticals and Industrials (ARI), Australian Nuclear Science \& Technology Organisation. 2003. 